Aus der Abteilung Mund-, Kiefer- und Gesichtschirurgie

(Prof. Dr. med. Dr. med. dent. H. Schliephake)

im Zentrum Zahn-, Mund- und Kieferheilkunde

der Medizinischen Fakultät der Universität Göttingen

\title{
Klinische Resultate sofort versorgter dentaler Implantate im Frontzahngebiet unter Anwendung kortikaler Mikrofixation
}

\author{
INAUGURAL-DISSERTATION
}

zur Erlangung des Doktorgrades

für Zahnheilkunde

der Medizinischen Fakultät

der Georg-August-Universität zu Göttingen

vorgelegt von

Victoria Elisabeth Rost

aus

Hamburg

Göttingen 2012 
Dekan: Prof. Dr. med. M. P. Schön

I. Berichterstatter: Prof. Dr. med. Dr. med. dent. W. Engelke II. Berichterstatter/in: PD Dr. med dent. D. Ziebolz III. Berichterstatter/in: Prof. Dr. med. M. Oppermann Tag der mündlichen Prüfung: 06.05.2014 


\section{Inhaltsverzeichnis}

1 Einleitung 1

1.1 Stand der Wissenschaft . . . . . . . . . . . . . . . 2

1.1.1 Versorgungsformen der Frontzahnlücke . . . . . . . . . . . . 2

1.1.2 Zeitpunkte der Versorgung und Belastung enossaler Implantate 3

1.1.3 Literaturübersicht Sofortversorgung von anterioren Implantaten 7

1.1.4 Aspekte der Frontzahnästhetik . . . . . . . . . . . . . . . . 12

1.1.5 Patientenzufriedenheit ................. 14

1.2 Das Konzept der Satellitenimplantate . . . . . . . . . . . . . . . . . . . 15

1.3 Zielsetzung der Arbeit . . . . . . . . . . . . . . . . . . . . . 17

2 Material und Methode $\quad 18$

2.1 Patientenkollektiv . . . . . . . . . . . . . . . . . . . . . . . 18

2.2 Versorgung der Patienten . . . . . . . . . . . . . . . . . . . . . . . 19

2.2 .1 Implantatsysteme . . . . . . . . . . . . . . . . . 19

2.2.2 Das Operationsverfahren . . . . . . . . . . . . 22

2.3 Evaluationen . . . . . . . . . . . . . . . . . . . . . . 23

2.3.1 Erfassung der Patientendaten und Ermittlung der Überlebensrate der Implantate . . . . . . . . . . . . . . . . . . . . 23

2.3.2 Klinische Nachuntersuchung . . . . . . . . . . . . . . . . . . 24

2.3.3 Radiologische Untersuchung . . . . . . . . . . . . 26

2.3.4 Erfolgskriterien ................... 28

2.3.5 Statistische Auswertung . . . . . . . . . . . . . 29

3 Resultate 31

3.1 Die Überlebensrate . . . . . . . . . . . . . . . . . . . . . . . . . . . 31

3.2 Beurteilung der Patientenzufriedenheit . . . . . . . . . . . . . . . . . 31

3.2.1 Beurteilung des Gesamtresultates der Behandlung . . . . . . 31

3.2 .2 Bewertung der Ästhetik . . . . . . . . . . . . . . . . 33

3.2.3 Bewertung der Abbeißfunktion . . . . . . . . . . . . . . 34

3.2.4 Bewertung der Phonetik . . . . . . . . . . . . . . . . 36

3.2.5 Bewertung der Hygienefähigkeit der Implantate . . . . . . . . . 37

3.2.6 Anzahl der Zahnarztbesuche aufgrund von Komplikationen . . . 38 
3.2.7 Nachfrage zur Behandlungsempfehlung . . . . . . . . . . . . . . 39

3.2.8 Nachfrage zur erneuten Behandlungswilligkeit . . . . . . . . . . 41

3.2.9 Nachfrage zur Zufriedenheit mit der Mundgesundheit . . . . . . 42

3.3 Die Risikofaktoren . . . . . . . . . . . . . . . . . . . . . . . . 44

3.4 Ergebnisse der Sondierungstiefen . . . . . . . . . . . . . . . 44

3.5 Blutungen auf Sondierung . . . . . . . . . . . . . 47

3.6 Papilla Presence Index . . . . . . . . . . . . . . . . . . . . . 48

3.7 Ergebnisse der Periotestmessung . . . . . . . . . . . . . . . . . 50

3.8 Ergebnisse der Bestimmung des Knochenabbaus / -gewinns . . . . . . 50

3.9 Die Erfolgsquote . . . . . . . . . . . . . . . . . 53

3.10 Falldokumentationen . . . . . . . . . . . . . . . . 53

4 Diskussion $\quad 58$

4.1 Beurteilung der Überlebensrate . . . . . . . . . . . . . . . . . . 58

4.2 Beurteilung der Patientenzufriedenheit . . . . . . . . . . . . . . . 59

4.3 Beurteilung der Sondierungstiefen und periimplantäten Gingiva . . . . 61

4.4 Beurteilung der Papillen . . . . . . . . . . . . . . . . . . 62

4.5 Beurteilung der Implantatstabilität . . . . . . . . . . . . . . . . 63

4.6 Beurteilung des Knochenabbaus / -gewinns . . . . . . . . . . . . . 65

4.7 Beurteilung der Erfolgsquote . . . . . . . . . . . . . . . . . . 67

4.8 Schlussfolgerung . . . . . . . . . . . . . . . . . . . . . 68

5 Zusammenfassung $\quad 69$

6 Anhang $\quad 71$

6.1 Abkürzungsverzeichnis . . . . . . . . . . . . . . . . . . . 71

6.2 Tabellenverzeichnis . . . . . . . . . . . . . . . . . . . . . . . 72

6.3 Abbildungsverzeichnis .................... 73

$\begin{array}{lll}7 & \text { Literaturverzeichnis } & 75\end{array}$ 


\section{Einleitung}

Nach einer Implantation besteht zunehmend der Wunsch der Patienten nach einer zügigen Wiederherstellung der Kaufunktion und Ästhetik. Insbesondere im Frontzahngebiet kann eine ästhetisch und funktionell unzureichende provisorische Versorgung die Lebensqualität der Patienten beeinträchtigen.

Das klassische von Brånemark (1983) aufgestellte Belastungsprotokoll für dentale Implantationen empfiehlt ein zweiphasiges operatives Vorgehen mit einer Einheilungszeit von 3 bis 6 Monaten. Erst nach dieser Einheilungszeit sei eine Belastung des dentalen Implantates durch eine prothetische Versorgung möglich (Brånemark (1983)). Trotz des gut dokumentierten Erfolges des von Brånemark propagierten Belastungsprotokolls wird fortwährend nach alternativen Behandlungsmethoden gesucht, um die Zahl der operativen Eingriffe zu reduzieren und die ästhetischen Ergebnisse zu optimieren.

Die sofortige Versorgung und Belastung von dentalen Implantaten ist im Bereich des zahnlosen Unterkiefers in der Literatur sehr gut dokumentiert. Zunehmend hat sich das Augenmerk jedoch auf die zügige Versorgung von Zahnlücken in der Oberkieferfrontzahnregion gerichtet. Die Herausforderung bei Implantationen in der Frontzahnregion besteht in der Erzielung eines ästhetisch ansprechenden Behandlungsergebnisses, welches maßgeblich durch den Verlauf des periimplantären Hart- und Weichgewebes beeinflusst wird. Viele Wissenschaftler betonen die Tatsache, dass durch eine Sofortversorgung nach Implantation das periimplantäre Gewebe besser erhalten wird (Harvey (2007), Valentini et al. (2010)). Andere Wissenschaftler erwähnen die potentiell höhere Verlustrate von enossalen Implantaten durch eine frühzeitige Belastung (Jivraj et al. (2005)).

Eine hohe Primärstabilität nach Implantation wird als Voraussetzung für eine erfolgreiche Osseointegration betrachtet (Meredith (1998)). Studien zeigten, dass das Ausmaß an Mikrobewegungen im Interface zwischen Implantat und Knochen während der Einheilphase die Osseointegration entscheidend beeinflusst (Meyer et al. (2004)). Je höher die Primärstabilität, desto geringer sind die Mikrobewegungen zwischen der Implantatoberfläche und dem umgebenden Knochen.

Das Konzept der Satellitenimplantate wurde entwickelt, um die Primärstabilität der dentalen Implantate zu erhöhen und eine sofortige Belastung der Implantate zu ermöglichen (Engelke et al. (2002)). Die Erhöhung der Primärstabilität von Implantaten in 
der Frontzahnregion verbessert die Voraussetzungen für Sofortimplantationen und Sofortversorgungen. Dabei wird womöglich nicht nur die Architektur des periimplantären Weichgewebes besser erhalten, sondern auch der Patientenkomfort durch die sofortige Versorgung erhöht.

\subsection{Stand der Wissenschaft}

\subsubsection{Versorgungsformen der Frontzahnlücke}

Zahnbegrenzte Lücken im Frontzahnbereich können durch Zahnverluste infolge von Karies, Parodontalerkrankungen und Traumata bedingt sein. Als Konsequenz liegt insbesondere im Frontzahnbereich meist nicht nur eine Einbuße der Ästhetik vor, sondern auch eine relevante Einbuße der Phonetik und Mastikation. Laut der DGZMK ist der Ersatz fehlender Zähne im Sinne einer Tertiärprophylaxe indiziert (Heydecke et al. (2008)).

Für die Versorgung von Frontzahnlücken stehen den Patienten unterschiedliche Therapieoptionen zur Verfügung. Hierzu zählen die konventionelle Brücke, die Adhäsivbrücke sowie implantatprothetische Versorgungsformen.

Konventionelle Brücken gehören in der Zahnheilkunde zu der Gruppe der am besten beschriebenen prothetischen Versorgungen. Im Allgemeinen werden bei der Versorgung mittels Brückenprothetik zufriedenstellende Resultate erzielt. Um die Ästhetik und Phonetik im Frontzahnbereich zu verbessern, können Brückenglieder mit eiförmiger Auflage (ovate pontic) gestaltet werden. Unter Berücksichtigung von biologischen und technischen Misserfolgen besitzen konventionelle Endpfeilerbrücken nach 10 Jahren eine Überlebenswahrscheinlichkeit von 87-89 \% (Tan et al. (2004)). Die Halbwertszeit wird derzeit auf rund 20 Jahre geschätzt (Kerschbaum (2004)). Der Verlust von Zahnhartsubstanz bei der Präparation einer Brücke mit allen bekannten Folgen sowie die erschwerte Parodontalhygiene im Bereich des Zwischengliedes sind als Nachteile dieser konventionellen Lückenversorgung zu erwähnen.

Als minimalinvasive Alternative zu herkömmlichen Brücken sind bei kariesfreien und weitgehend füllungsfreien Pfeilerzähnen im Frontzahnbereich Adhäsivbrücken indiziert. Diese werden mittels Befestigungskunststoffen an die säurebehandelte Schmelzoberfläche von Pfeilerzähnen geklebt. In einigen Studien haben sich Adhäsivbrücken als weniger zuverlässig erwiesen als konventionelle Brücken (Kerschbaum et al. (1996), Kohlmeyer et al. (2004), Stark et al. (1994)). Diese Studien stellten fest, dass sich nach 5 Jahren etwa $25 \%$ der Adhäsivbrücken an mindestens einem Pfeiler gelöst hatten. Mittlerweile konnten allerdings andere Studien zeigen, dass bei der Anwendung 
einer retentiven Präparationsform und eines adäquaten klinischen Vorgehens die Überlebensrate von Adhäsivbrücken auf $95 \%$ nach 6 bis 10 Jahren gesteigert werden konnte (Rammelsberg et al. (1995), Behr et al. (1998)) und damit etwa auf dem Niveau konventioneller Brücken liegt (Kerschbaum (2004)).

$\mathrm{Zu}$ den minimalinvasiven Techniken zählen auch Einzelzahnimplantationen, die ein Beschleifen der Nachbarzähne überflüssig machen. Die Versorgung mit implantatgetragenen Kronen stellt eine erfolgsversprechende Alternative zu den genannten Versorgungsformen dar. So zeigen viele Studien gute Langzeitergebnisse bei der Versorgung des teilbezahnten Patienten (Richter (2005)). Festsitzender implantatgetragener Zahnersatz bedeutet für den Patienten nicht nur eine Erhöhung des Tragekomforts, sondern bietet auch gute Voraussetzungen für eine möglichst ästhetische und natürliche Gestaltung des Zahnersatzes. Neben der hohen Funktionalität liegen die Vorteile der Implantation in der langfristig knochenerhaltenden Lagerung. Jung et al. (2008) schätzten die Überlebensrate von Einzelzahnimplantaten nach 5 Jahren auf 94,5 \%. Ähnliches ergab auch die Metaanalyse von Pjetursson und Lang. Sie schätzten die Überlebensrate von Einzelzahnimplantaten nach 10 Jahren auf 89,4 \% (Pjetursson und Lang (2008)). Somit liegt die Überlebenswahrscheinlichkeit für implantatgetragenen Einzelzahnersatz nach 10 Jahren zwischen 80 und 90 \% (Holm-Pedersen et al. (2007)), was etwa der Bewährung herkömmlicher Brücken entspricht.

\subsubsection{Zeitpunkte der Versorgung und Belastung enossaler Implantate}

Enossale Implantate können nach ihrer Insertion in den Kieferknochen zu unterschiedlichen Zeitpunkten durch Zahnersatz belastet werden. Es gibt viele Versuche, die Begrifflichkeiten bezüglich der Belastungszeiten von dentalen Implantaten zu definieren.

Gemäß der dritten ITI-Konsensus-Konferenz, die 2003 in Gstaad stattfand, wurden die Begriffe Sofortbelastung, Sofortversorgung, frühe Belastung, konventionelle Belastung und späte Belastung wie folgt definiert (Cochran et al. (2004)):

Sofortbelastung: Der Zahnersatz wird innerhalb von 48 Stunden nach Implantation mit okklusalem Kontakt zum Antagonisten eingegliedert.

Sofortversorgung: Eingliederung des Zahnersatzes innerhalb von 48 Stunden ohne okklusalen Kontakt zum Antagonisten.

Frühe Belastung: Eingliederung des Zahnersatzes mit okklusalem Kontakt nach frühestens 48 Stunden und bis spätestens 3 Monaten nach Implantation. 
Konventionelle Belastung: Der Zahnersatz wird nach einer Einheilzeit der Implantate von 3 bis 6 Monaten eingegliedert.

Späte Belastung: Der Zahnersatz wird nach einer Einheilzeit der Implantate von mehr als 3 bis 6 Monaten eingegliedert.

Auf der ersten Europäischen Konsensus-Konferenz Implantologie (EuCC) erarbeiteten Wissenschaftler den „Kölner Konsens 2006“ (Berger (2006)). Sie definierten die Sofortbelastung als die Eingliederung des Zahnersatzes mit okklusalem Kontakt zum Antagonisten bis 72 Stunden nach der Implantation. Davon grenzte die EuCC die Sofortversorgung ab, bei der der Zahnersatz zwar im gleichen Zeitfenster eingegliedert wird, aber keinen okklusalen Kontakt zum Antagonisten aufweist.

In der Literatur sind bezüglich der Definition der Sofortbelastung Abweichungen zu denen der EuCC oder ITI-Konsensus- Konferenz zu finden. Randow et al. (1999) definierten die Eingliederung eines Zahnersatzes auch 10 Tage nach Implantation als Sofortbelastung. Hingegen haben Achilli et al. (2007) die Sofortbelastung als Eingliederung des Zahnersatzes innerhalb von 24 Stunden nach Implantation definiert.

Die Studien der letzten Jahre zeigen, dass die Sofortbelastung oder Sofortversorgung von Implantaten eine Alternative zu dem konventionellen, zweiphasigen Vorgehen darstellen. Bei dem konventionellen Vorgehen heilen die Implantate zunächst unbelastet unter der Schleimhaut oder auf Schleimhautniveau ein. Danach werden sie freigelegt und mit dem Zahnersatz versorgt. Die Einheilungszeit von 3 bis 6 Monaten nach der Implantation soll eine Osseointegration des Implantates gewährleisten und eine Minimierung der Implantatverlustrate bewirken. Dieses zweizeitige Verfahren wird klinisch schon seit Jahren erfolgreich angewendet (Brånemark et al. (1995), Buser et al. (1997)). Der Wunsch nach einer schnelleren implantatgetragenen Versorgung hat dazu geführt, dass die Sofortbelastung und Sofortversorgung von Implantaten immer mehr an Bedeutung gewinnen. Die sofortige Versorgung eines Implantates ist dabei nicht synonym mit einer funktionellen Belastung zu verstehen. Wenn das Provisorium keine okklusalen Kontakte aufweist können Einzelzahnversorgungen zunächst funktionell weitgehend unbelastet provisorisch versorgt werden (Schliephake (2004)).

Eine gute Primärstabilität kann als wichtigstes Kriterium für eine erfolgreiche Osseointegration nach Implantation betrachtet werden (Meredith (1998)). Studien zeigten, dass das Ausmaß an Mikrobewegungen im Interface zwischen Implantat und Knochen während der Einheilphase die Osseointegration ausschlaggebend beeinflusst (Meyer et al. (2004)). Wenn also die Stabilität nach Implantation ausreichend ist, kann im teilbezahnten Unter- und Oberkiefer eine sofortige oder verzögerte Belastung von Implantaten angestrebt werden (Weber et al. (2009)).

Der Zeitpunkt der Implantation ist natürlich auch für die Primärstabilität des Implan- 
tates und die Qualität des knöchernen Lagers entscheidend. Bei einer Spätimplantation wird das Implantat in ein allseits knöchern konsolidiertes Lager inseriert, während bei der Sofortimplantation das Implantat in eine frische Extraktionsalveole platziert wird. Bei einer Spät- und einer Sofortimplantation liegen also unterschiedliche Bedingungen vor. Deshalb werden Sofortversorgungen nach Spätimplantation von denen nach Sofortimplantation unterschieden (Schliephake (2004)).

Viele Studien verglichen bereits einerseits die Behandlungsresultate der Sofort- und der Spätimplantation und andererseits die Resultate der unterschiedlichen Versorgungsund Belastungszeiten von Implantaten in der anterioren Oberkieferregion.

Chaushu et al. (2001) verglichen in einer Studie die Überlebensrate von Einzelzahnimplantaten nach Sofortimplantation und Spätimplantation. 26 Patienten erhielten 28 sofort versorgte Einzelzahnimplantate. Davon wurden 19 Implantate in frische Extraktionsalveolen und 9 in abgeheilte Alveolen inseriert. Die Nachuntersuchung fand 6 bis 24 Monate nach Insertion statt. Die Überlebensrate der Implantate nach Sofortimplantation betrug 82,4 \% und nach Spätimplantation 100 \%. Trotz der höheren Misserfolgsrate betrachteten die Autoren die Sofortimplantation als alternative Behandlungsmöglichkeit zur Spätimplantation.

In einer prospektiven Studie von Malo et al. (2003) wurden 116 Implantate in der ästhetisch sichtbaren Region sofort nach Implantation provisorisch versorgt. Die Provisorien wiesen keine okklusalen Kontakte auf. Nach 6 Monaten wurde der definitive Zahnersatz eingegliedert. Nach einem Jahr betrug die Überlebensrate 95,7 \%.

86 Patienten mit guter Knochenqualität wurden von Locante (2004) für eine Studie ausgewählt. Die frischen Extraktionsalveolen in der Oberkiefer-Frontzahnregion wurden mit Einzelzahnimplantaten versorgt. Diese wurden einer sofortigen funktionellen Belastung ausgesetzt. Nach 3 Jahren betrug die Überlebensrate 98,8 \%.

In einer Studie von Ribeiro et al. (2008) wurden 64 Patienten im anterioren Oberkiefer mit Einzelzahnimplantaten versorgt. 46 Implantate wurden in frische Extraktionsalveolen inseriert und 36 in abgeheilte Alveolen. Die Implantate wurden sofort provisorisch versorgt. Das Beobachtungsintervall lag zwischen 18 und 39 Monaten. Die Überlebensrate der Implantate betrug bei der Sofortimplantation 93,5\% und bei der Spätimplantation $100 \%$.

In einer prospektiven Studie von Crespi et al. (2008) wurden 40 Patienten behandelt. Nach Sofortimplantation im Oberkiefer-Frontzahnbereich wurden 20 Implantate sofort belastet (Versuchsgruppe) und 20 Implantate erst nach 3 Monaten belastet (Kontrollgruppe). Nach 2 Jahren betrug die Überlebensrate aller Implantate 100 \%. Die Versuchsgruppe wies einen mittleren marginalen Knochenverlust von 1,02 +/- 0,51 mm und die Kontrollgruppe einen von 1,16 +/- 0,51 mm auf, ein signifikanter Unterschied zwischen beiden Gruppen konnte also nicht festgestellt werden. 
Mijiritsky et al. (2009) untersuchten 16 Patienten mit 24 sofort versorgten Implantaten nach Sofortimplantation in der Oberkiefer-Frontzahnregion. Nach 6 Jahren betrug die Überlebensrate 95,8 \% . Der mittlere Knochenverlust betrug 0,9 +/- 1,1 mm.

Die Ermittlung einer 5 Jahres-Überlebensrate für sofort versorgte Implantate war das Ziel einer Studie von Laviv et al. (2010). An der Nachuntersuchung nahmen 113 Patienten mit sofort versorgten Implantaten und 113 Patienten mit konventionell belasteten Implantaten teil. Die Implantate waren vorwiegend in der Frontzahnregion lokalisiert. Insgesamt betrug die Überlebensrate der Implantate nach 5 Jahren 96,5 \%. Es wurden keine statistisch signifikanten Unterschiede zwischen der Versuchs- und der Kontrollgruppe beobachtet.

Valentini et al. (2010) behandelten 40 Patienten im Bereich des anterioren Oberkiefers. Die Einzelzahnimplantate wurden innerhalb einer Woche nach Sofortimplantation mit provisorischen Kronen versorgt, wobei keine okklusalen Kontakte vorhanden waren. Nach 1 Jahr betrug die Überlebensrate 95,3 \%, kein statistisch signifikanter marginaler Knochenverlust konnte festgestellt werden und bei $78 \%$ der Patienten waren die Papillen vollständig erhalten.

In einer systematischen Literaturübersicht von Esposito et al. (2010) zum Thema des Zeitpunktes der Implantation nach Zahnextraktion vertreten die Autoren den Standpunkt, dass die Evidenzlage für die möglichen Vor- und Nachteile der Spät- oder Sofortimplantation unzureichend sei. Einerseits werde in der Literatur vorgeschlagen, dass Sofortimplantate anfälliger für Komplikationen seien, andererseits könne das Behandlungsergebnis ästhetisch ansprechender sein als das nach Spätimplantation. Einig ist man sich jedoch, dass eine hohe Primärstabilität die Voraussetzung für einen Implantationserfolg nach sofortiger Versorgung bzw. Belastung von Implantaten ist (Esposito et al. (2007)). 


\subsubsection{Literaturübersicht Sofortversorgung von anterioren Implantaten}

Im Rahmen dieser Studie wurde eine Literaturrecherche durchgeführt, in der die medizinische online-Datenbank medline durchsucht wurde. Ziel war es, sich einen Überblick über den aktuellen Stand der Wissenschaft bezüglich sofort versorgter Implantate im Frontzahngebiet zu verschaffen.

Die Suche wurde mit folgenden Begriffen durchgeführt:

- anterior implants immediate loading: 134 Treffer.

- (a)esthetic zone implants immediate loading: 18 / 19 Treffer.

- anterior implants immediate non occlusal loading: 5 Treffer.

- anterior implants immediate provisionalization: 29 Treffer.

- (a)esthetic zone implants immediate provisionalization: 8/15 Treffer.

Die Ergebnisse der oben genannten Suchbegriffe wurden anhand folgender Kriterien weiter eingeschränkt:

- Klinische Studien von 2005 bis Ende 2011.

- Patientenanzahl von mehr als 10 Patienten.

- (Einzelzahn-) Implantate in der anterioren Ober- und Unterkieferregion.

- Sofortversorgung / -belastung.

Die Resultate der berücksichtigten klinischen Studien werden in der folgenden Tabelle präsentiert. 


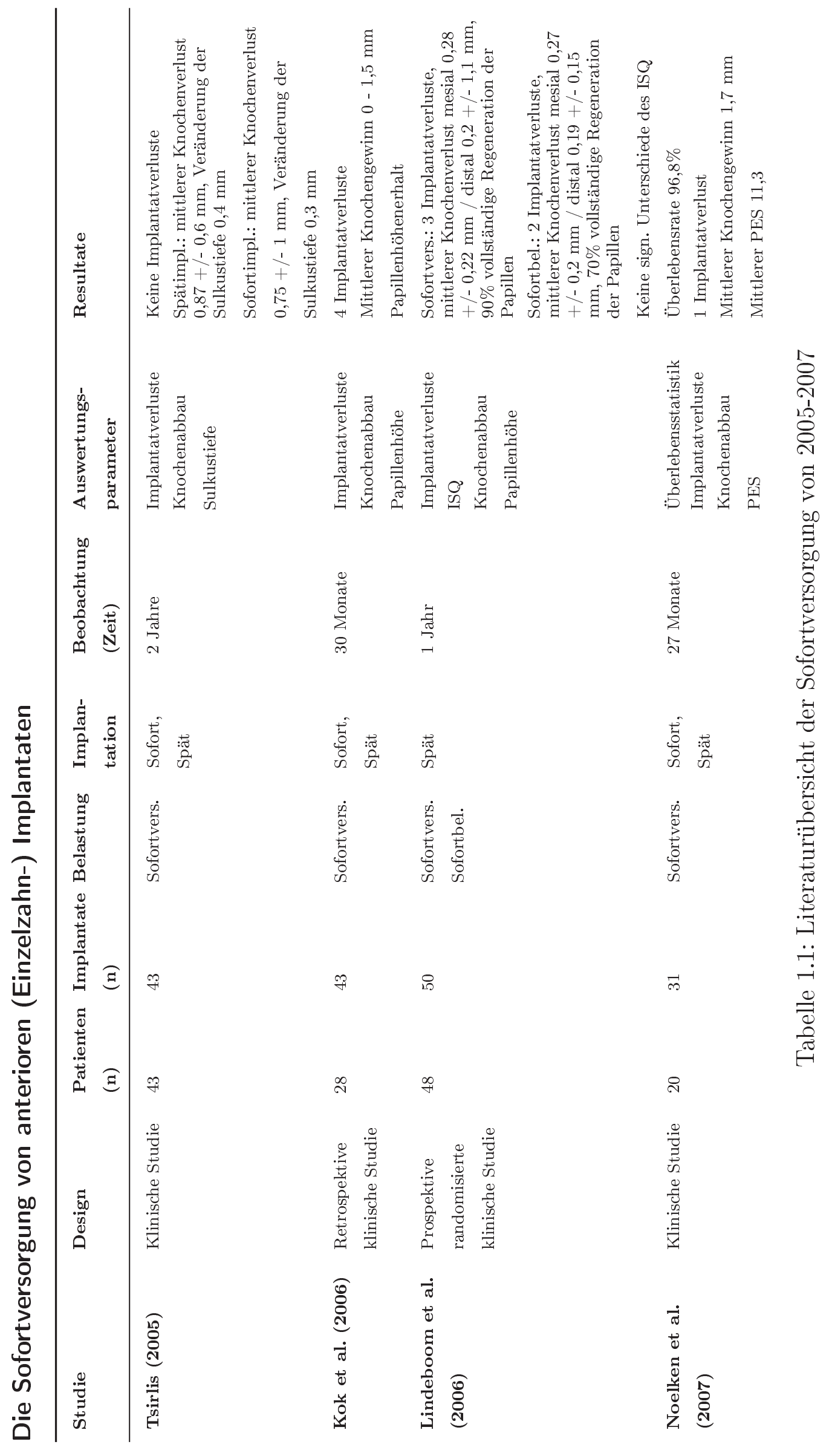




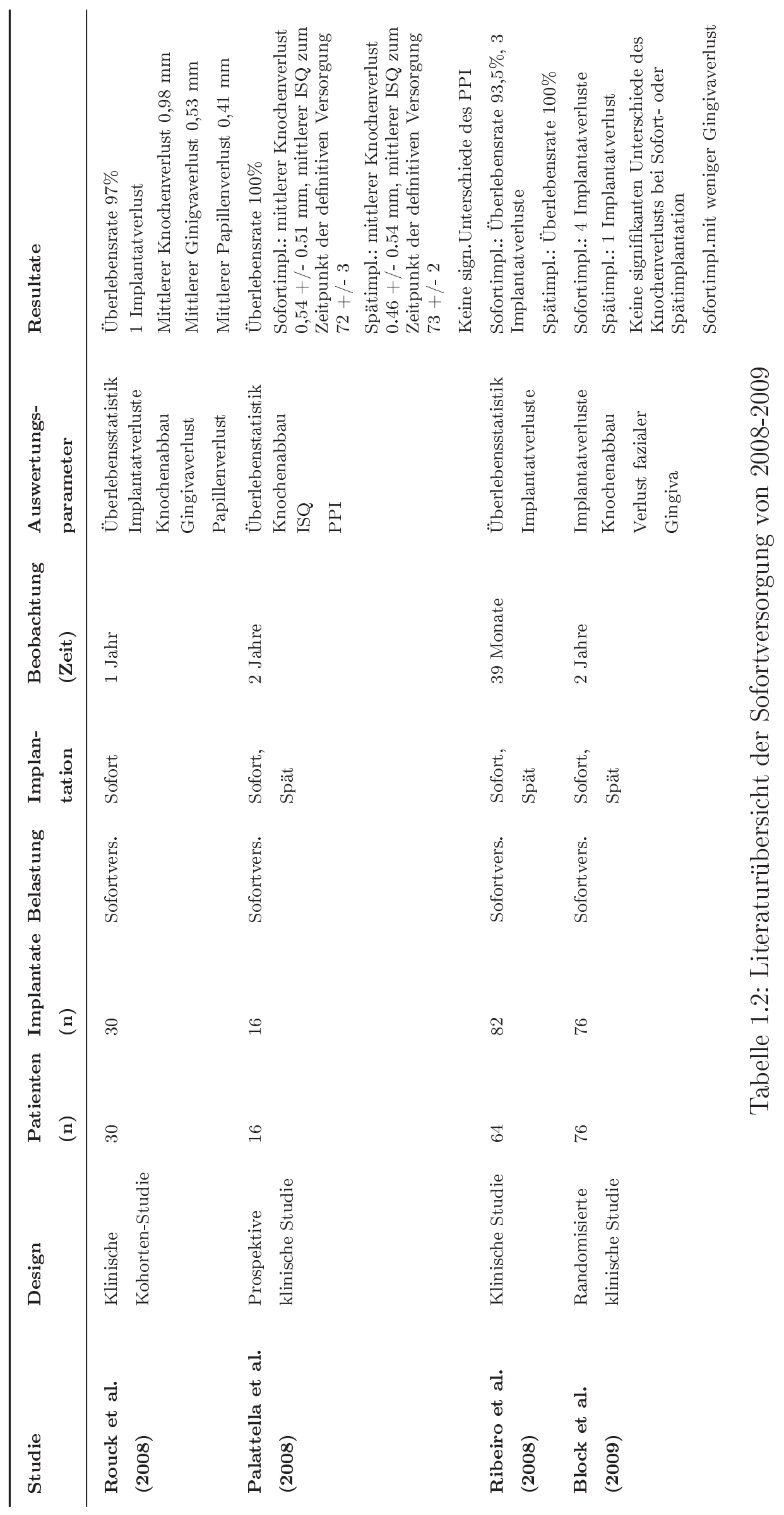




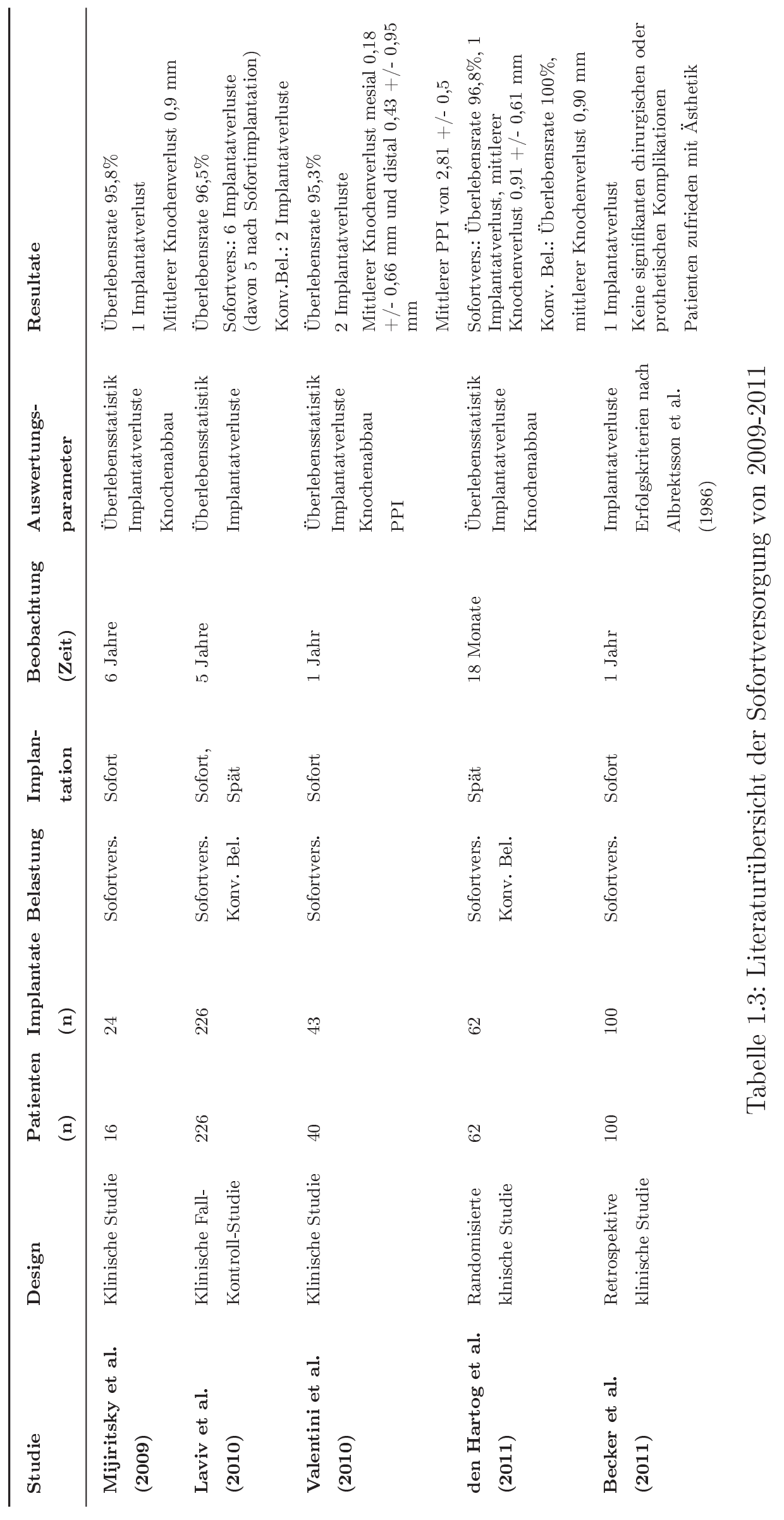




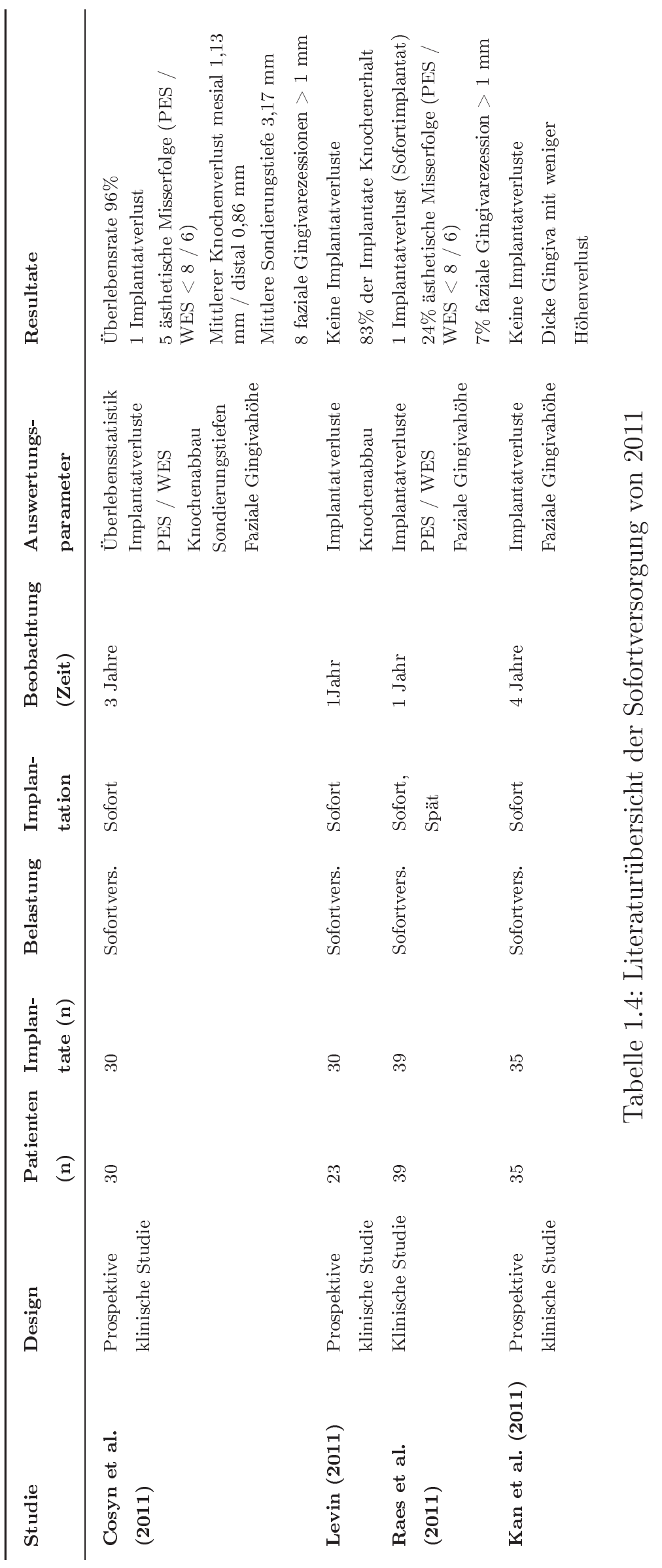




\subsubsection{Aspekte der Frontzahnästhetik}

Der Verlust von Zähnen kann lokale, ästhetische und psychische Auswirkungen zur Folge haben. Dabei können vor allem ästhetische Defizite im Frontzahnbereich die Lebensqualität von Betroffenen erheblich beeinträchtigen (Elias und Sheiham (1998)).

Allgemein anerkannt ist, dass die Ästhetik von Frontzähnen und Frontzahnimplantaten nicht nur durch das Erscheinungsbild der Zahnkronen, sondern auch durch das des umgebenden Weichgewebes beeinflusst wird. Insbesondere dem Erhalt der interdentalen Papille und dem darunter liegenden Alveolarknochen wird eine zentrale Rolle bei der Erzielung eines langfristig ästhetischen Implantationsergebnisses zugeschrieben.

Zahlreiche Publikationen beschäftigen sich aus diesem Grunde mit dem Thema der Ästhetikoptimierung bei Frontzahnimplantationen. Sie geben allgemeine Überblicke zur Patientenauswahl, der Beurteilung der lokalen Situation, dem chirurgischen und prothetischen Vorgehen sowie möglichen Komplikationen bei der Implantation (Jivraj und Chee (2006), Belser et al. (2004) und Buser et al. (2004)).

Andere Publikationen betonen die Bedeutung der Sofortimplantation bei dem Erhalt der Konturen des periimplantären Weich- und Hartgewebes (Valentini et al. (2010), Harvey (2007), Sammartino et al. (2007), Canullo und Rasperini (2007), Wheeler et al. (2000), Locante (2001)). Nur durch eine sofortige Versorgung des Implantates mit einem Provisorium sei eine Optimierung der roten Ästhetik möglich (Rouck et al. 2009).

Besonderes Gewicht legen andere Arbeiten auf die Positionierung des Implantates im Zahnbogen sowie zu benachbarten Zähnen und Implantaten (Gomez-Roman (2001), Wheeler (2007), Bedard (2009)). Die Autoren beschreiben bestimmte Vorgehensweisen bei der Implantatinsertion, die dem Erhalt der interdentalen Papille und des periimplantären Gewebes dienen sollen.

Die Implantateinbringung ohne Lappenbildung stellen wiederum andere Autoren in den Vordergrund (Oh et al. (2007), Turkyilmaz und Suarez (2009), Koutrach und Nimmo (2010)). Minimalinvasive chirurgische Techniken seien nicht nur in Hinblick auf den Erhalt des periimplantären Gewebes, sondern auch auf die Minimierung der postoperativen Beschwerden vorteilhaft. Gaggl et al. (2002) kamen zu dem Schluss, dass die transmukosale Stanzung hinsichtlich der Ästhetik und des Papillenerhaltes zu guten Ergebnissen führt.

Juodzbalys und Wang (2010) betrachten die Morphologie der Extraktionsalveole als Schlüssel zur roten Ästhetik. Anhand dieser sei der individuell optimale Zeitpunkt der Implantation zur Erzielung eines ästhetischen Ergebnisses bestimmbar.

Darüber hinaus werden in der Literatur verschiedene prothetische Behandlungskonzepte vorgeschlagen. Gemeinsames Ziel ist das klinische Bild einer Papille, die den interdentalen Raum ausfüllt (Belser et al. (2004), Buser et al. (2008), Holst et al. (2005), 
Kourtis et al. (2007), Locante (2004)). Zu nennen ist hier das Konzept des sogenannten platform switchings (Baumgarten et al. (2005)). Bei dem platform switching wird der Übergang zwischen Implantat und Aufbauteil (Abutment) weiter nach zentral verlagert. Durch den Versatz des Implantat-Abutment-Interfaces vom Knochen weg sollen mechanische und mikrobielle Reize vom periimplantären Gewebe ferngehalten und eine bessere Anlagerung des periimplantären Gewebes ermöglicht werden.

Im Großen und Ganzen ist man sich zwar einig, dass die Osseointegration bei der Implantation im Frontzahnbereich vorhersehbar, aber der Erhalt oder die Wiederherstellung der periimplantären Ästhetik oft nur schwer erreichbar ist. Der Fragestellung, was im Rahmen der Implantologie als ästhetisch empfunden wird, widmen sich weniger Studien.

Vereinzelt haben Wissenschaftler Indizes entwickelt, welche bei der Evaluation der periimplantären Ästhetik des Implantationsergebnisses helfen sollen.

Jemt (1997) entwickelte den Papilla Presence Index (PPI). Der PPI unterscheidet 5 verschiedene Grade von Papillenhöhen und wird jeweils mesial und distal der Implantate erhoben:

0 : Papille nicht vorhanden.

1 : Papille füllt weniger als $1 / 3$ des interdentalen Dreiecks aus.

2 : Papille füllt bis zu 2/3 des interdentalen Dreiecks aus.

3 : Papille füllt das interdentale Dreieck vollständig aus.

4: Papille ist hypertrophisch.

Seit der Entwicklung des PPI wendeten auch andere Autoren diesen Index an. Somanathan et al. (2007) stellten in einer Studie unter Anwendung des PPI in Kombination mit röntgenologischen Untersuchungen fest, dass die Papillenhöhe und die interproximale Alveolarkammhöhe sich proportional zueinander verhalten. Ein PPI von 2 oder 3 weist also auf einen guten Erhalt des periimplantären Weich- und Hartgewebes sowie eine ansprechende rote Ästhetik hin.

Fürhauser et al. (2005) entwickelten den Pink Esthetic Score (PES) zur Evaluation der periimplantären Weichgewebsverhältnisse um Einzelzahnimplantate. Man gab 20 Untersuchern (5 Prothetikern, 5 Oralchirurgen, 5 Kieferorthopäden und 5 Zahnmedizinstudenten) Fotoaufnahmen von 30 prothetisch versorgten Einzelzahnimplantaten. Die mesiale und distale Papille, das Niveau und die Kontur des Weichgewebes, die Alveolarkammhöhe sowie die Farbe und Struktur des Weichgewebes wurden mit einem natürlichen Referenzzahn verglichen. Für diese 7 Variablen definierte man eine Bewertungsskala von 0 bis 2, wobei 0 für den schlechtesten und 2 für den besten Wert stehen. Ein maximaler PES von 14 ist also erreichbar. Die aus der Beurteilung resultierende 
Summe beschreibt somit die Qualität des ästhetischen Resultates. Je größer der PES, desto „schöner“ wirkt das Behandlungsergebnis. Die Autoren stellten jedoch fest, dass diese Bewertungsmethode abhängig von der Disziplin des Betrachters war. So erwiesen sich die Kieferorthopäden als die kritischsten Bewerter.

Letztendlich ist die rote Ästhetik trotz der Entwicklung dieser Indizes nicht gänzlich objektivierbar. Viel wichtiger als die Meinung der Wissenschaftler ist natürlich, ob der Patient seine Implantatversorgung als ästhetisch empfindet.

\subsubsection{Patientenzufriedenheit}

Die Verbesserung der Lebensqualität und des oralen Wohlbefindens der Patienten steht mehr den je im Blickpunkt der Implantologie (Locker (1998)). Das Erkennen und Erfassen der Patientenzufriedenheit kann als elementar für die Bewertung des oralen Wohlbefindens und somit auch für die Bewertung des Erfolges einer zahnärztlichen Versorgung betrachtet werden (Buch et al. (2002)). Aus diesem Grunde haben viele Studien, auch durch die Verwendung von Fragebögen, versucht die Zufriedenheit der Patienten zu erfassen.

Gibbard und Zarb (2002) nutzten bei der Beurteilung des Behandlungserfolges von Einzelzahnimplantaten einen Fragebogen. Mit der Hilfe dieses Fragebogens wollten sie die Patientenzufriedenheit erfassen. Sie stellten Fragen zum Aussehen der Implantatversorgung, zur Funktion, zur Hygienefähigkeit, zur Weiterempfehlung der Behandlung sowie zur erneuten Behandlungswilligkeit. Als Antwortoptionen standen 4 Möglichkeiten, beginnend bei „sehr zufrieden“ oder „empfehlenswert“ und endend bei „sehr unzufrieden“ oder „nicht empfehlenswert“, zur Verfügung.

Meijndert et al. (2007) nutzten visuelle Analogskalen bei der Erfragung der Patientenzufriedenheit. Dabei reichten die Skalen von 0-10. Werte ab 6 wurden als Behandlungserfolg gewertet.

In einer Literaturübersicht von Belser et al. (2004) werden verschiedene Studien erwähnt, die ebenfalls Fragebögen zur Erfassung der Zufriedenheit der Patienten mit implantologischen Versorgungen im Frontzahnbereich verwendeten. Die häufigsten Fragen galten der Zufriedenheit mit der (Abbeiß-) Funktion, Ästhetik, Phonetik sowie dem generellen Behandlungsergebnis. Darüber hinaus wurden die Patienten auch gefragt, ob sie einem guten Bekannten die Behandlung empfehlen und sich selbst nochmals der Behandlung unterziehen würden.

Die international anerkannten Erfolgskriterien dentaler Implantate nach Jahn und d'Hoedt (1992) beinhalten auch die Bewertung des Behandlungsergebnisses durch den Patienten. Dabei beschlossen die Autoren, dass eine Implantatversorgung in Bezug auf 
die Patientenzufriedenheit als Erfolg gewertet werden darf, wenn die Patienten dem Gesamtbehandlungsergebnis keine schlechtere Schulnote als 3 („befriedigend“) geben.

\subsection{Das Konzept der Satellitenimplantate}

Als ,Satellitenimplantat" bezeichnet man ein Hilfsimplantat in Form einer Osteosyntheseschraube. Diese Hilfsimplantate werden verwendet, um die Primärstabilität von dentalen Implantaten zu erhöhen, d.h. jene Stabilität, die direkt nach der Insertion vorhanden ist.

Das Konzept der Satellitenimplantate wurde von Engelke et al. (2002) entwickelt. Die eigentlichen dentalen Implantate werden dabei als so genannte „Zentralimplantate" bezeichnet. Die Verbindung der Osteosyntheseschrauben zum Zentralimplantat wird über subgingivale Mikroosteosyntheseplatten hergestellt. Diese Konnektoren sind am Implantataufbauteil befestigt. Die Abbildung 1.1 veranschaulicht das Prinzip der Satellitenimplantate anhand eines Oberkiefer-Frontzahnimplantates mit 2 palatinalen Osteosyntheseschrauben. Durch die kortikale Mikrofixation mittels der Osteosyntheseschrauben sollen die nach palatinal fortgeleiteten auftreffenden Kaukräfte abgefangen werden.

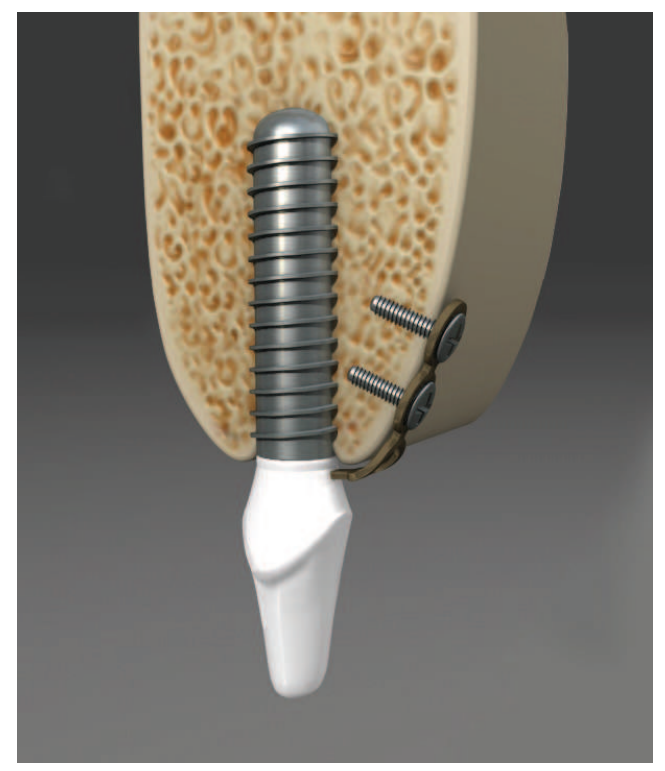

Abbildung 1.1: Frontzahn-Satellitenimplantat

2002 wurden in einem Laborversuch die Auswirkungen von Satellitenimplantaten auf die Primärstabiliät der eigentlichen dentalen Implantate untersucht. Unmittelbar nach Zahnentfernung wurden Implantate in die Seitenzahnregion von Schweinekiefern inseriert. Vor und nach sowohl vestibulärer als auch lingualer Einbringung einer Osteosyntheseschraube wurden Periotestwerte erhoben. Die Implantate waren über die am 
Implantataufbau befestigten Mikroosteosyntheseplatten mit den Osteosyntheseschrauben verbunden. Die Autoren stellten fest, dass die horizontale Stabilität der Implantate durch die Verwendung von Satellitenimplantaten erhöht wird (Engelke et al. (2002)). Eine gute Primärstabilität kann als Voraussetzung für eine erfolgreiche Osseointegration betrachtet werden und stellt besonders im Hinblick auf eine prothetische Sofortversorgung einen wichtigen Parameter für die Implantatprognose dar (Meredith (1998)). Meyer et al. (2004) stellten fest, dass das Ausmaß an Mikrobewegungen im Interface zwischen Implantat und Knochen während der Einheilungsphase die Osseointegration entscheidend beeinflusst. Mit Hilfe der zusätzlichen Verankerung von Osteosyntheseschrauben in der Kortikalis wird die Primärstabilität des Implantates deutlich erhöht und das Auftreten von Mikrobewegungen verringert. Eine höhere Primärstabilität ermöglicht wiederum eine frühere funktionelle Belastung. Die Anwendung der kortikalen Mikrofixation mittels Satellitenimplantaten erlaubt es also unter bestimmten Bedingungen, ein Implantat unmittelbar nach der Zahnextraktion einzubringen und unverzüglich mit einem Provisorium zu versorgen.

Das Konzept des subgingivalen Verbundes des Implantates mit kortikalen Osteosyntheseschrauben ist mit dem Konzept der primären Verblockung von Implantaten durch supragingivale Stegkonstruktionen vergleichbar. Beide haben das Ziel, eine Sofortbelastung zu ermöglichen, und beide haben bereits erfolgreiche Resultate hervorgebracht (Ledermann (1979), Engelke et al. (2011)). So veröffentlichten Engelke et al. (2011) Ergebnisse einer prospektiven Studie, an der 20 zahnlose Patienten teilnahmen. Die Patienten erhielten jeweils 2 Satellitenimplantate in der Regio interforaminalis, welche unverzüglich mit einer Deckprothese belastet wurden. Nach einem Beobachtungszeitraum von 36 Monaten lag die Überlebensrate bei 100 \%. Es konnte bei allen Implantaten eine erfolgreiche Osseointegration festgestellt werden.

Obwohl eine hohe Primärstabilät als Voraussetzung für eine Sofortversorgung betrachtet wird (Rouck et al. (2009)) und sich zahlreiche Studien mit der Sofortversorgung von Einzelzahnimplantaten in der anterioren Oberkieferregion beschäftigen, befassen sich wenige mit den Möglichkeiten zur Verbesserung der Primärstabilität dieser Implantate. Danza et al. (2010) vertreten den Standpunkt, dass durch ein bestimmtes Implantatdesign auch bei Sofortimplantationen mit Sofortversorgungen eine ausreichende Primärstabiltät erzielt werden kann. Die meisten Autoren betonen einfach die Notwendigkeit der Vermeidung von okklusalen Kräften (Berger (2006), Wang et al. (2006)).

Der Wunsch nach einer schnelleren und ästhetisch anspruchsvollen implantatgetragenen Versorgung hat in den letzten Jahren dazu geführt, dass die Sofortversorgung von Implantaten insbesondere im Frontzahnbereich immer mehr an Bedeutung gewinnt. Um eine Sofortversorgung zu ermöglichen müssen dentale Implantate jedoch stabil verankert sein. Laut dem 3. ITI- Konsensus ist eine Sofortbelastung in der ästhetisch 
sichtbaren Zone des teilbezahnten Kiefers nur möglich, wenn die erreichte Primärstabilität ausreichend ist (Weber et al. (2009)). Mit Hilfe von Satellitenimplantaten kann die notwendige Implantatstabilität möglicherweise auch unter erschwerten Bedingungen erreicht und eine Sofortversorgung ermöglicht werden.

\subsection{Zielsetzung der Arbeit}

Im Rahmen dieser retrospektiv angelegten Studie soll der klinische Erfolg des Konzepts der Sofortversorgung von dentalen Implantaten im Frontzahngebiet unter Anwendung kortikaler Mikrofixation bewertet werden. Dabei sollen die dentalen Implantate hinsichtlich der folgenden Parameter evaluiert werden:

- Überlebensrate der sofort versorgten Implantate.

- Patientenzufriedenheitsanalyse mittels einer Befragung.

- Beurteilung des Zustandes der periimplantären Gingiva/Papillen.

- Beurteilung der Implantatstabilität durch Bestimmung der Periotestwerte.

- Beurteilung des periimplantären horizontalen Knochenabbaus bzw. -gewinns.

- Erfolgsquote der sofort versorgten Implantate.

- Differenzierung der Resultate hinsichtlich des Implantationszeitpunktes.

- Darstellung eventueller Komplikationen und Probleme. 


\section{Material und Methode}

\subsection{Patientenkollektiv}

In dieser retrospektiven Studie wurden Patienten mit Implantaten konsekutiv untersucht, bei denen die Indikation für eine Implantatversorgung im Oberkieferfrontzahnbereich bestand, die den Wunsch nach einer Sofortversorgung äußerten und bei denen die unten aufgeführten Ein- und Ausschlusskriterien beachtet wurden.

Einschlusskriterien:

- Patienten, bei denen eine Indikation für die Versorgung einer Zahnlücke im Frontzahnbereich mit dentalen Implantaten bestand.

- Patienten mit abgeschlossenem Längenwachstum.

- Patienten mit gutem Allgemeinzustand.

- Patienten mit qualitativ und quantitativ ausreichendem knöchernem Implantatlager.

Ausschlusskriterien:

- Patienten mit schweren Allgemeinerkrankungen und reduziertem Allgemeinzustand, die eine Lokalanästhesie oder einen zahnärztlich chirurgischen Eingriff von längerer Dauer (ca. 1 Stunde) ausschließen.

- Patienten während oder nach Therapie mit Bisphosphonaten.

- Raucher mit einem regelmäßigen Konsum von 20 oder mehr Zigaretten pro Tag.

- Patienten mit unbehandelter Parodontitis.

- Patienten mit ausgeprägter Funktionsstörung (Bruxismus).

Die Implantationen sowie die späteren Nachuntersuchungen im Zeitraum von März bis Juli 2011 fanden in der Abteilung Mund-, Kiefer- und Gesichtschirurgie der Universitätsmedizin Göttingen und in der Praxis für zahnärztliche Chirurgie von Dr. Möller in Göttingen statt.

An dieser Studie nahmen nach einem mittleren Beobachtungszeitraum von 46 Monaten (Min. 7 Monate; Max. 130 Monate; Std.abw. 26 Monate) 44 Patienten (Durchschnittsalter 58 Jahre; Min. 23 Jahre; Max. 90 Jahre; Std.abw. 14,3 Jahre) teil. 
Unter den Patienten waren 29 Frauen und 15 Männer. Jeder Patient in dieser Studie erhielt mindestens 1 und maximal 2 Implantate in der Regio 013-023. Die 44 Patienten erhielten insgesamt 52 Implantate.

Von diesen 44 Patienten konnten 35 (23 Frauen und 12 Männer; 40 Implanate) nachuntersucht und befragt werden. Bei den restlichen 9 Patienten (6 Frauen und 3 Männer; 12 Implantate) war nur eine telefonische Befragung möglich, da sie fortgezogen waren oder diese Nachuntersuchung ablehnten.

In der Sofortimplantationsgruppe befanden sich 31 Patienten (20 Frauen und 11 Männer) mit einem durchschnittlichen Alter von 57 Jahren. Diese Gruppe umfasste insgesamt 33 Sofortimplantate.

13 Patienten (9 Frauen und 4 Männer) mit einem Durchschnittsalter von 62 Jahren befanden sich in der Gruppe der Spätimplantationen. Diese Gruppe umfasste insgesamt 19 Spätimplantate.

Das Patientenkollektiv wird in der nachfolgenden Tabelle 2.1 nochmals veranschaulicht.

$\begin{gathered}\text { Teilnehmende Patienten } \mathbf{n}=44(\text { Implantate } \mathbf{n}=\mathbf{5 2}) \\ \text { Nachuntersuchung \& Befragung }\end{gathered}$
Patienten $\mathrm{n}=35($ Implantate $\mathrm{n}=40)$
Sofortimplantate $\mathrm{n}=24 \mid$ Spätimplantate $\mathrm{n}=16$

Tabelle 2.1: Veranschaulichung des Patientenkollektivs

\subsection{Versorgung der Patienten}

\subsubsection{Implantatsysteme}

Im Zuge dieser Studie wurden verschiedene Implantate der Firma Straumann Freiburg/ Deutschland sowie das Semadosimplantat der Firma Bego Bremen/ Deutschland verwendet. Bei allen verwendeten Implantaten handelt es sich um Schraubenimplantate aus unlegiertem Titan.

In der Universitätsmedizin Göttingen erhielten die Patienten 8 Semadosimplantate (siehe Abb. 2.1). Das Semadosimplantat besitzt eine konische Grundform. Die Gewindetiefe nimmt dabei sukzessiv von der Implantatschulter nach apikal hin zu. Laut 
Hersteller führt dies zur verbesserten Knochenkontaktrate und somit erhöhten Implantatstabilität. Durch die Mikrostrukturierung der Oberfläche besitzt das Semadosimplantat osteokonduktive Eigenschaften. Bei der Verbindung zwischen Implantat und prothetischem Aufbau handelt es sich um einen Innenkonus mit Sechskant.

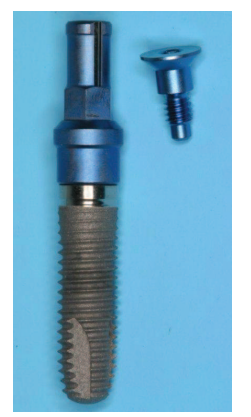

Abbildung 2.1: Semados Implantat (Malinski (2011) S.21)

In der Praxis von Dr. Möller erhielten die Patienten Implantate der Firma Straumann. Darunter waren 4 Standard Implantate, 15 Standard Plus Implantate und 25 Bone Level Implantate (siehe Abb. 2.2).

Die verwendeten Straumann-Implantate unterscheiden sich hinsichtlich Länge, Durchmesser und Geometrie. Dabei verfügen sie alle über eine sogenannte SLA- Oberfläche (Sand-blasted, Large-grit, Acid-etched). Diese durch Sandstrahlen und Säureätzung geschaffene Oberflächenstruktur besitzt osteokonduktive Eigenschaften und erlaubt eine frühe Belastung des Implantats (Cochran et al. (1998)), Buser und von Arx et al. (2000)). Bei der Verbindung zwischen Implantat und prothetischem Aufbau handelt es sich um eine Schraubekonusverbindung mit Innenachtkant.

Das zylindrische Straumann Standard Implantat besitzt eine Schulterhöhe von 2,8 mm mit einem zervikal polierten Abschnitt und Tulpenform. Das Standard Plus Implantat hingegen besitzt einen kürzeren 1,8 mm zervikal polierten Abschnitt und kann aus diesem Grunde tiefer in den Knochen inseriert werden. Durch die weiter subgingival liegende Implantatschulter sind laut Straumann ästhetisch ansprechende Restaurationen einfacher realisierbar. Das Straumann Bone Level Implantat wird bis auf Knochenniveau inseriert. Es besitzt eine zylindrische äußere Kontur, wobei es im koronalen Teil des Implantats leicht konisch wird. Laut Hersteller bietet das Bone Level Implantat eine erhöhte vertikale Flexibilität und unterstützt den Erhalt des krestalen Knochens, was in ästhetisch anspruchsvollen Fällen vorteilhaft ist. 


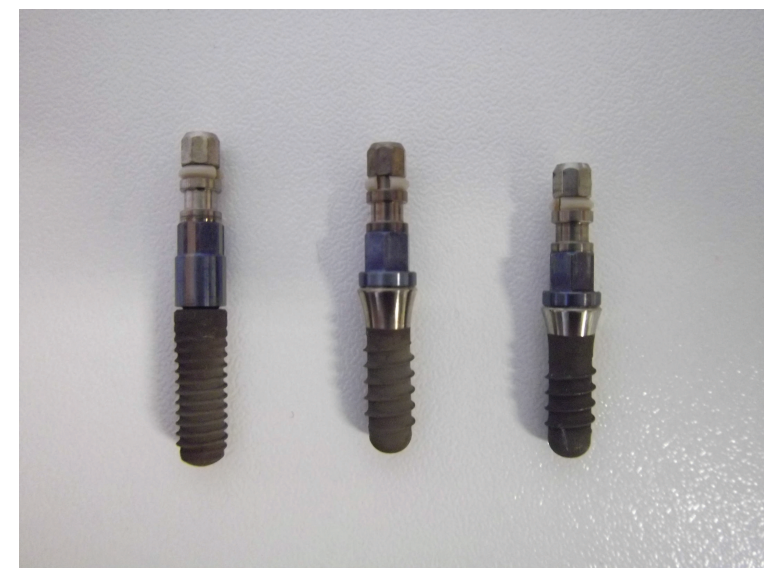

Abbildung 2.2: Straumann Implantate (Bone Level, Standard, Standard Plus)

In der nachfolgenden Tabelle (siehe Tab. 2.2) werden die Charakteristika der einzelnen Implantattypen nochmals veranschaulicht.

\begin{tabular}{lllll} 
Implantat & Form & Verbindung & Gewinde & Oberfläche \\
\hline Semados & Konus, polierter & Innenkonus, & Nach apikal zunehmende Steigung & Geätzt \\
& Halsabschnitt $0,5 \mathrm{~mm}$ & Sechskant & & \\
\multirow{2}{*}{ Bone Level } & Zylinder, kein polierter & Innenkonus, & Konstante Steigung $0,8 \mathrm{~mm}$ & Sandgestrahlt, geätzt \\
& Halsabschnitt & Achtkant & & \\
\multirow{2}{*}{ Standard } & Zylinder, polierter & Innenkonus, & Konstante Steigung $1 \mathrm{~mm}$ & Sandgestrahlt, geätzt \\
& Halsabschnitt $2,8 \mathrm{~mm}$ & Achtkant & & \\
\multirow{2}{*}{ Standard Plus } & Zylinder, polierter & Innenkonus, & Konstante Steigung $1 \mathrm{~mm}$ & Sandgestrahlt, geätzt \\
& Halsabschnitt $1,8 \mathrm{~mm}$ & Achtkant & & \\
\hline
\end{tabular}

Tabelle 2.2: Charakteristika der verwendeten Implantate

Implantatdurchmesser von 3,3 $\mathrm{mm}, 3,75 \mathrm{~mm}$ und 4,1 $\mathrm{mm}$ wurden verwendet. Die Implantatlängen betrugen $10 \mathrm{~mm}, 12 \mathrm{~mm}, 14 \mathrm{~mm}, 16 \mathrm{~mm}$ oder $18 \mathrm{~mm}$.

Bei den eigentlichen Satellitenimplantaten handelte es sich um Osteosyntheseschrauben aus Titan mit einem Durchmesser von 1,7 mm in den Längen $4 \mathrm{~mm}, 5 \mathrm{~mm}, 6 \mathrm{~mm}$, $7 \mathrm{~mm}$ oder $8 \mathrm{~mm}$. Die Patienten erhielten im Rahmen der kortikalen Mikrofixation mindestens eine und maximal zwei Ostheosyntheseschrauben. Als Satellitenimplantatkonnektoren dienten 3-Loch-Ostheosyntheseplatten aus Titan (siehe Abb. 2.3). Die Satellitenimplantatkonnektoren wurden im zahntechnischen Labor aus den von Hersteller zertifizierten Komponenten durch Laserverschweißung individuell vor der Implantation an den Abutments befestigt und anschließend sterilisiert (siehe Abb. 2.4a). Die verwendeten Osteosyntheseschrauben und -platten stammten in der Universitätsmedizin 
Göttingen von der Firma Medartis Basel/ Schweiz und in der Praxis von Dr. Möller von der Firma Mondeal Mühlheim/ Deutschland.

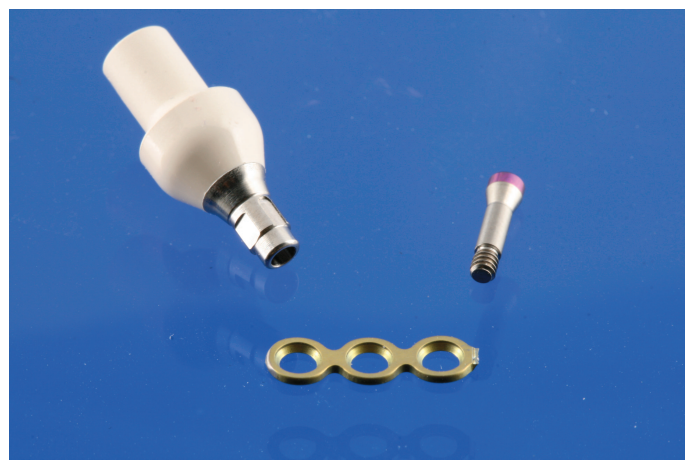

Abbildung 2.3: Satellitenimplantatabutment und Konnektor (Dr. Möller)

\subsubsection{Das Operationsverfahren}

Die chirurgischen Eingriffe wurden unter Lokalanästhesie in den Operationsräumen der Abteilung Mund-, Kiefer- und Gesichtschirurgie der Universitätsmedizin Göttingen sowie in der Praxis für zahnärztliche Chirurgie von Dr. Möller in Göttingen durchgeführt.

Die Implantationen fanden entweder unverzüglich in einer Operationssitzung mit der Zahnentfernung (Sofortimplantation) oder nach vollständiger Abheilung des alveolären Zahnfaches statt (Spätimplantation).

Die Implantation mit kortikaler Mikrofixation begann mit einer krestalen Inzision mit palatinaler Entlastung, sodass die Präparation eines Mukoperiostlappens ermöglicht wurde. Die benachbarte und antagonistische Bezahnung diente als prothetische Referenz bei der Festlegung der Position und Ausrichtung der Implantate. Entsprechend den Richtlinien des Implantatherstellers erfolgte die Aufbereitung der Implantatlager mit Hilfe von Bohrern. Die Bohrungen fanden unter Einsatz des Operationsmikroskopes statt, sodass auffällige Befunde inspiziert werden konnten. Die Zentralimplantate wurden juxtaalveolär auf dem Niveau des krestalen Knochens inseriert. Die am Implantatabutment befestigten Satellitenimplantatkonnektoren wurden mittels einer Zange an die jeweilige individuelle palatinale Knochenmorphologie angepasst (siehe Abb. 2.4a und 2.4b). Das Implantatabutment selbst wurde so im Zentralimplantat fixiert, dass sich der Satellitenimplantatkonnektor palatinal befand. Nach der Aufbereitung der Satellitenimplantatlager im Bereich der Löcher des Konnektors wurden die Satellitenimplantate palatinal inseriert. Die Insertion wurde unter Spülung mit physiologischer Kochsalzlösung durchgeführt. Der Wundverschluss erfolgte mit Einzelknopfnähten. 


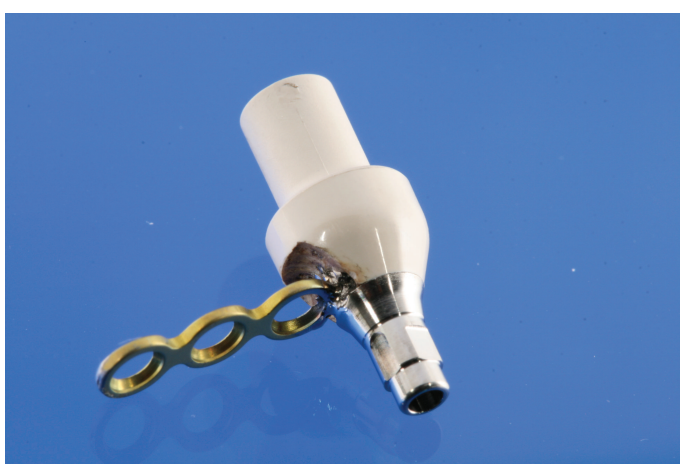

(a) Konnektor gerade

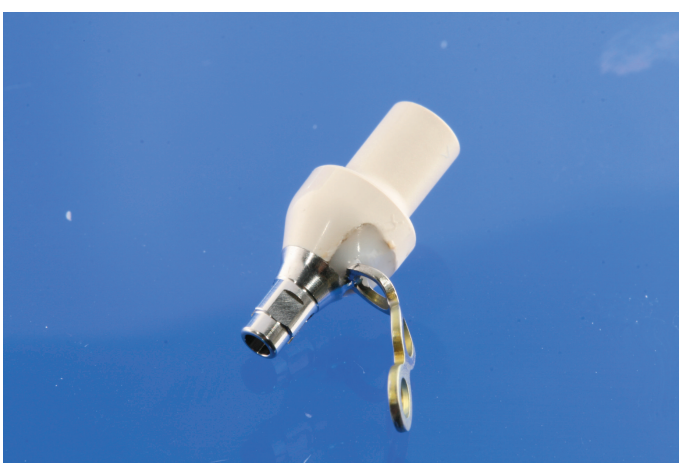

(b) Konnektor gebogen

Abbildung 2.4: Provisorisches Abutment mit befestigten Konnektor (Dr. Möller)

Postoperativ wurde eine Röntgenaufnahme angefertigt und die Implantate wurden mit provisorischen Kronen versorgt. Es wurde darauf geachtet, dass die Provisorien keine statischen okklusalen Kontakte aufwiesen. Nach 7 bis 10 Tagen konnten die Fäden entfernt werden.

3 bis 6 Monate nach dem operativen Eingriff konnten die Satellitenimplantate unter Lokalanästhesie entfernt werden (siehe Abb. 2.5). Die definitive prothetische Versorgung konnte nach Entfernung der Satellitenimplantate von den jeweiligen Hauszahnärzten der Patienten eingegliedert werden. Dabei erhielten die Patienten Einzelzahnkronen, Brückenversorgung oder verblockte Kronen.

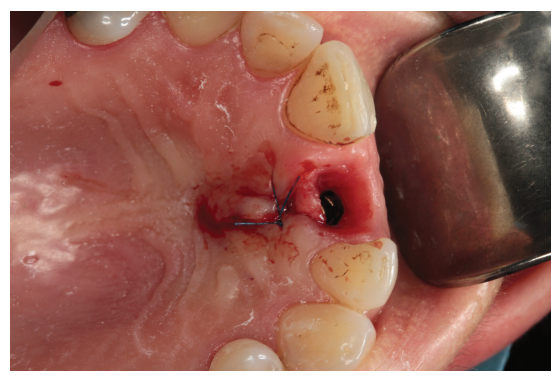

Abbildung 2.5: Zustand nach Entfernung eines Satellitenimplantates (Dr. Möller)

\subsection{Evaluationen}

\subsubsection{Erfassung der Patientendaten und Ermittlung der Überlebensrate der Implantate}

Nach Erhalt eines positiven Ethikvotums und Erfassung der Patientendaten und Implantatangaben aus den Archiven der Praxis für zahnärztliche Chirurgie von Dr. Möller in Göttingen sowie der Abteilung Mund-, Kiefer- und Gesichtschirurgie der Universitätsmedizin Göttingen wurden die Patienten angeschrieben und telefonisch kontaktiert. 
Bei der telefonischen Kontaktaufnahme wurde zunächst gefragt ob sich die Implantate ohne Beschwerden in situ befinden. Darüberhinaus wurde erfragt, ob die Patienten an der klinischen Nachuntersuchung teilnehmen wollen. Bei einer Absage, aber einer Bereitwilligkeit den Fragebogen zur Patientenzufriedenheitsanalyse zu beantworten wurden die Patienten anschließend telefonisch befragt.

Anhand der Daten der telefonischen Befragung und klinischen Nachuntersuchung soll die Überlebensrate der Implantate ermittelt werden.

\subsubsection{Klinische Nachuntersuchung}

Vor Beginn der Nachuntersuchung wurde der Untersucher bezüglich der Reproduzierbarkeit der zu erhebenden Parameter kalibriert. In Anlehnung an Zafiropoulos et al. (2010) wurde die Kalibrierung des Untersuchers durch einen erfahrenen Mitarbeiter der Abteilung für Mund-, Kiefer- und Gesichtchirurgie der Universitätsmedizin Göttingen durchgeführt.

Die klinische Untersuchung umfasste die Einverständniserklärung und Befragung der Patienten, die Ermittlung der Sondierungstiefen, die Erhebung des Indices PPI, die Bestimmung des Periotestwertes sowie die Anfertigung von Röntgenaufnahmen sofern diese im Rahmen der regulären Implantatnachsorge indiziert waren.

\section{Patientenfragebögen}

Zur Erfassung der Patientenzufriedenheit und des oralen Wohlbefindens wurde ein Fragebogen der Arbeitsgruppe der Abteilung Mund-, Kiefer- und Gesichtschirurgie der Universitätsmedizin Göttingen modifiziert und verwendet (Malinski (2011)). Der Fragebogen bestand aus neun Fragen, von denen 5 durch eine Benotung nach dem deutschen Schulnotensystem und 3 durch Ja/Nein beantwortet werden sollten. Eine Frage wurde offen gestellt.

1. Frage zur Zufriedenheit des Gesamtresultates: Wie beurteilen Sie das Gesamtresultat Ihrer Behandlung?

2. Frage zur Ästhetik des Zahnersatzes: Wie schätzen Sie die Ästhetik Ihrer implantatgetragenen Versorgung ein?

3. Frage zur Abbeißfunktion: Wie schätzen Sie Ihre Abbeißfunktion ein?

4. Frage zur Phonetik: Wie schätzen Sie Ihre Sprachfunktion ein?

5. Frage zur Hygienefähigkeit der Implantate: Haben/ hatten Sie Probleme bei der Reinigung Ihrer Implantate? 
6. Frage zu Komplikationen/Problemen mit dem Implantat: Wie oft mussten Sie aufgrund von Problemen oder Beschwerden mit Ihrem Implantat einen Zahnarzt aufsuchen? (bitte die Anzahl der Besuche eintragen)

7. Frage zur Behandlungsempfehlung: Würden Sie die Behandlung einem guten Bekannten weiterempfehlen?

8. Frage zur erneuten Behandlungswilligkeit: Würden Sie bei erneuten Behandlungsbedarf wieder eine Implantatbehandlung wählen?

9. Frage zur Mundgesundheit: Sind Sie zufrieden mit Ihrer Mundgesundheit?

Darüber hinaus sollten die Fragen 1., 2., 3., 4., 7., 8. und 9. auf einen weiteren Fragebogen anhand visueller Analogskalen beantwortet werden. Die visuellen Analogskalen bestanden jeweils aus einer $10 \mathrm{~cm}$ langen horizontalen Linie, auf der die Punkte 0, 5 und $10 \mathrm{~cm}$ kenntlich gemacht wurden. Die Endpunkte wurden durch mögliche Antworten auf die gestellte Frage ergänzt, wobei der Punkt 0 der größtmöglichen Unzufriedenheit und der Punkt $10 \mathrm{~cm}$ der größtmöglichen Zufriedenheit entsprach. Beim Auswerten wurde die Strecke vom Punkt 0 bis zum jeweils vom Patienten markierten Punkt in mm ausgemessen. Die Beantwortung des Fragebogens fand entweder telefonisch oder im Rahmen der klinischen Nachuntersuchung statt.

\section{Beurteilung der periimplantären Gingiva/Papillen}

Der Zustand der periimplantären Gingiva wurde mit Hilfe einer millimeterskalierten Parodontalsonde der Firma HuFriedy Rotterdam/ Niederlande (Typ XP23/ UNC 15) ermittelt. Mit der Parodontalsonde konnten die Taschentiefen mesial, vestibulär, distal und palatinal der Implantate bestimmt werden. Gemessen wurde von dem tiefsten Punkt der periimplantären Tasche bis zum oberen Rand der marginalen Gingiva. Im Untersuchungsprotokoll wurden die erhobenen Werte in Millimetern dokumentiert. Die statistische Auswertung wurde mit den jeweiligen Messdaten der einzelnen Sondierungpositionen durchgeführt.

Des Weiteren wurden beim Sondieren auftretende Blutungen im Untersuchungsprotokoll als positiv oder negativ vermerkt, d.h. es wurde nicht differenziert ob es an einem oder mehreren Messpunkten zu einer Blutung kam.

Das Ausmaßs der mesialen und distalen periimplantären Papille wurde mit Hilfe der 5 Grade des von Jemt (1997) entwickelten Papilla Presence Index (PPI) beurteilt. Hierbei stehen der Grad 0 für das Nichtvorhandensein einer Papille und der Grad 4 für das Vorhandensein einer hypertrophischen Papille. 


\section{Bestimmung der Periotestwerte}

Zur Bestimmung der Stabilität und Osseointegration der Implantate wurde das PeriotestVerfahren verwendet. Es handelt sich dabei um ein nichtinvasives elektromechanisches Messverfahren, welches von W. Schulte entwickelt wurde (d'Hoedt et al. (1985)).

Das Periotestgerät besitzt einen Stößel, welcher impulsartig auf das zu überprüfende Implantat auftrifft und dabei abgebremst wird. Innerhalb eines Messvorgangs (4 Sekunden) trifft der Stößel 16- mal auf das Implantat. Je fester das Implantat im Knochen verankert ist, desto kürzer ist die Kontaktzeit zwischen Stößel und Implantat. Die bei der Messung vom Periotestgerät wahrgenommenen Kontaktzeiten werden bestimmten Werten zwischen -8 und +50 zugeordnet. Negative Werte stehen dabei für eine hohe und positive Werte für eine geringe Stabilität des Implantates.

Bei der Nachuntersuchung wurde das Periotestgerät der Firma Medizintechnik Gulden Bensheim/ Deutschland verwendet. Besonderes Augenmerk wurde darauf gelegt, dass die Periotestwerte horizontal zum Implantat bestimmt wurden und dass der korrekte Messabstand zum Implantat eingehalten wurde. Die erhobenen Messwerte wurden im Untersuchungsprotokoll notiert.

\subsubsection{Radiologische Untersuchung}

Zum Zeitpunkt der Nachuntersuchung wurden die im Rahmen der üblichen Implantatnachsorge vorhandenen aktuellen Röntgenaufnahmen ausgewertet. In der Praxis von Dr. Möller handelte es sich hierbei um Einzelzahnfilme und in der Universitätsmedizin Göttingen um OPAN Aufnahmen. Voraussetzung für die Verwendung der Aufnahmen war die Darstellung des Implantates in voller Länge sowie des umgebenden periimplantären Knochens. Röntgenaufnahmen mit erkennbaren technischen Fehlern wurden von der Studie ausgeschlossen.

\section{Bestimmung des periimplantären Knochenabbaus/-gewinns}

Für die Zustandsbeschreibung der osseären Integration eines Implantates ist die radiologische Darstellung des periimplantären Knochens essenziell. Voraussetzung ist dabei die Festlegung eines implantatspezifischen Referenzpunktes. Als Referenzpunkte wurden im Rahmen dieser Studie der Implantathals sowie der tiefste Punkt des horizontalen Knochenabbaus/-gewinns festgelegt.

Die Vermessung der Röntgenaufnahmen wurde in Anlehnung an Borg et al. (2002) und Park (2010) mit Hilfe des Programmes Sidexis XG der Firma Sirona Bensheim/ 
Deutschland vorgenommen. Die auf den Röntgenaufnahmen dargestellte Implantatlänge wurde anhand der dokumentierten realen Länge der Implantate überprüft (siehe Abb. 2.6).

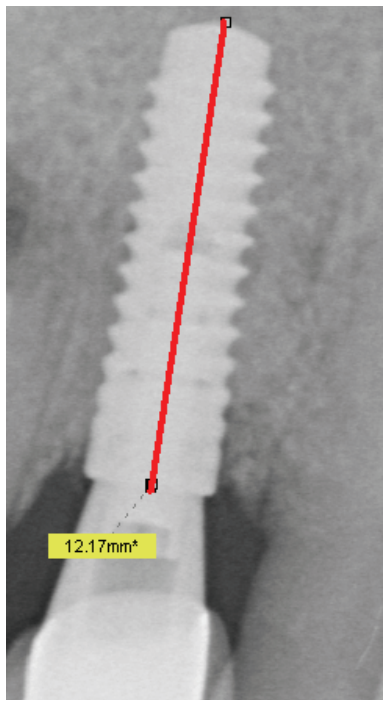

(a) Implantatlänge

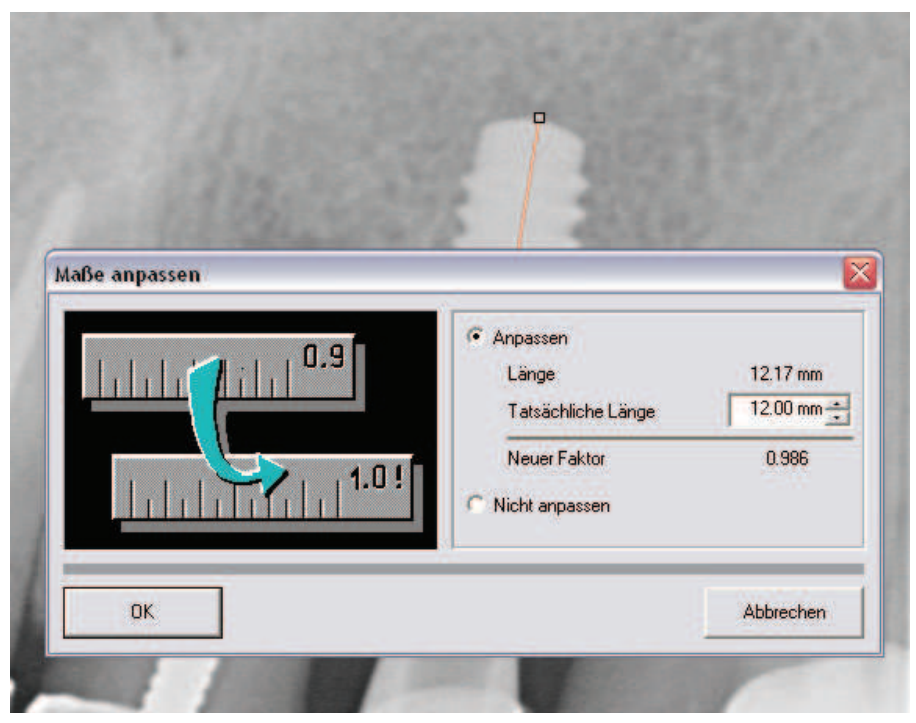

(b) Reale Implantatlänge

Abbildung 2.6: Berücksichtigung der individuellen Dimensionsänderung

Dabei wurde darauf geachtet, dass bei den Straumann Standard und Standard Plus Implantaten die Länge des Halses zur Gesamtlänge des Implantates hinzuaddiert wurde. Bei den Semadosimplantaten und Bone Level Implantaten war dies nicht nötig, da der Implantathals der Implantatschulter entspricht. Anschließend wurde der Abstand des Implantathalses vom periimplantären Knochen mesial und distal unter Berücksichtigung der ermittelten Dimensionsänderung bestimmt (siehe Abb. 2.7). Die mesial und distal erhobenen Messwerte wurden im Untersuchungsprotokoll notiert.

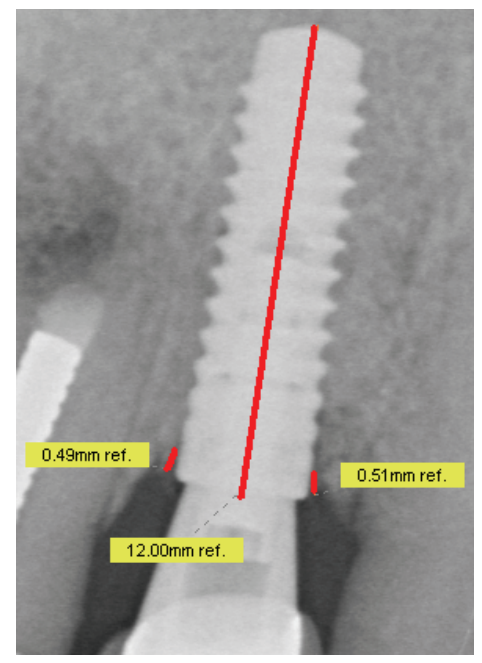

Abbildung 2.7: Bestimmung des mesialen und distalen Knochenabbaus 
Es wurden sowohl die postoperative Röntgenaufnahme als auch die aktuelle Röntgenaufnahme der Nachuntersuchung vermessen. Dadurch konnte bestimmt werden, ob seit dem Zeitpunkt der Implantation ein Knochenverlust oder -gewinn stattgefunden hat. Zur statistischen Analyse wurde der jeweilige Mittelwert der mesialen und distalen Messung gebildet und verwendet.

\section{Bestimmung lokaler Risikofaktoren}

Mit Hilfe der zum Zeitpunkt der Implantation hergestellten Röntgenaufnahmen wurden lokale Risikofaktoren, die möglicherweise das Implantationsergebnis beeinflussen könnten, bestimmt. Notiert wurden, ob die Nachbarzähne endodontisch behandelt waren, einen apikalen Herd oder einen erweiterten Parodontalspalt aufwiesen. Darüberhinaus wurde der Abstand der Schmelzzementgrenze oder des prothetischen Kronenrandes der Nachbarzähne zum Limbus alveolaris mit Hilfe des Computerprogrammes Sidexis XG (Sirona Bensheim/ Deutschland) in mm ausgemessen. Auf diese Weise konnte man feststellen, ob die Nachbarzähne schon zum Zeitpunkt der Implantation einen Knochenverlust vorzuweisen hatten.

\subsubsection{Erfolgskriterien}

Anhand von bestimmten Erfolgskriterien soll die Erfolgsrate der Implantate ermittelt werden. Um den Zustand der periimplantären Weich- und Hartgewebsparameter zu berücksichtigen, wurden, wie auch in den anderen Implantatstudien der Arbeitsgruppe der Abteilung Mund-, Kiefer- und Gesichtschirurgie der Universitätsmedizin Göttingen, die Erfolgskriterien nach Jahn und d'Hoedt (1992) und Naert et al. (1992)/ Snauwaert et al. (2000) verwendet (siehe Tabelle 2.4). 


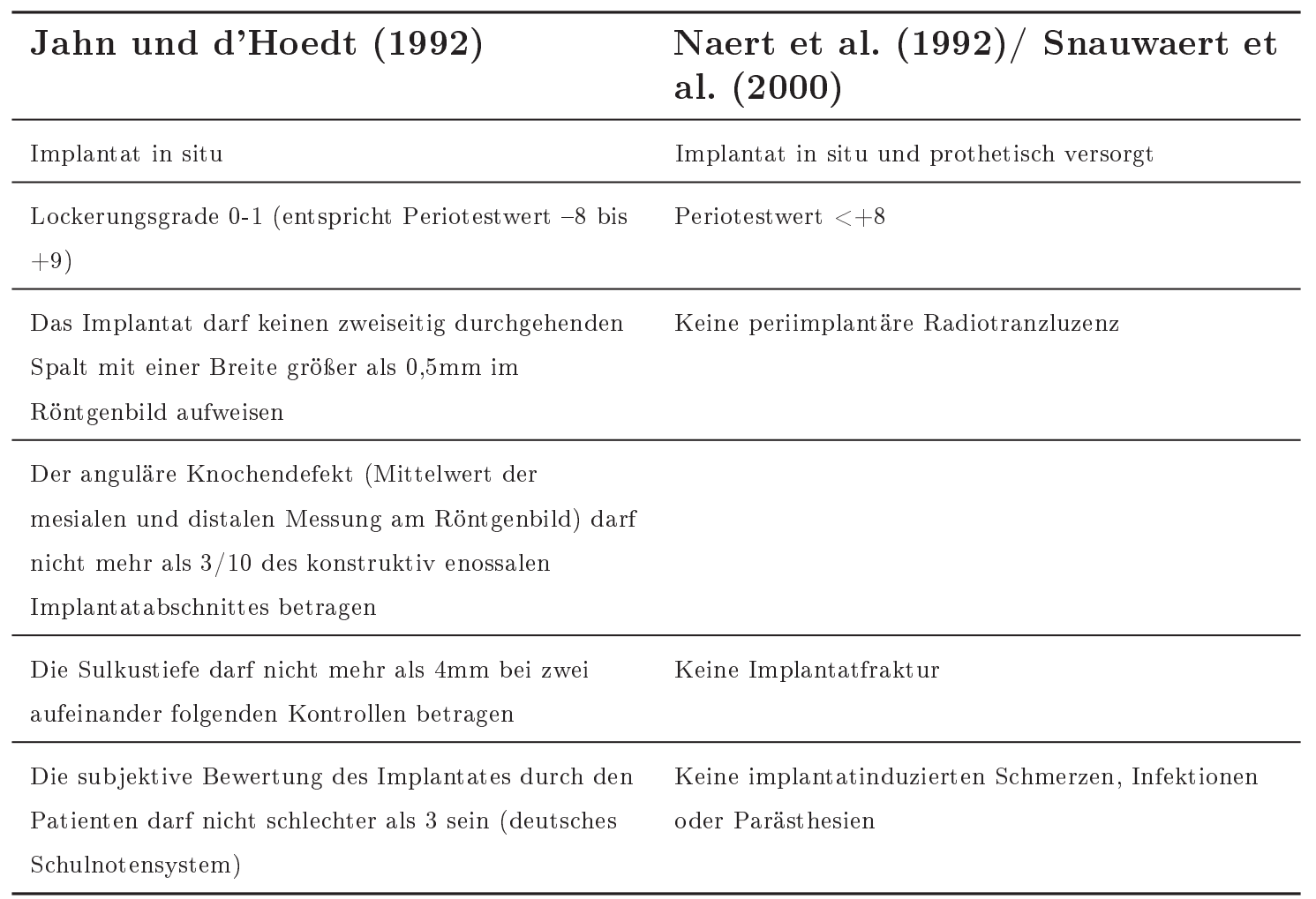

Tabelle 2.4: International anerkannte Erfolgskriterien für enossale dentale Implantate (Malinski (2011) S. 24)

\subsubsection{Statistische Auswertung}

Die statistische Auswertung wurde mit Hilfe der Programmen Statistika 10.0 und Microsoft Office Excel 2007 durchgeführt.

Für die deskriptive Auswertung wurden abhängig von der Zielvariable entweder Anzahl und Prozente bei kategorialen Variablen (z.B. Blutung auf Sondierung), Mittelwerte und Standardabweichung bei metrischen Größen (z.B. Knochenabbau) oder Median und Quantile bei ordinalen Daten (z.B. PPI) angegeben.

Für die graphische Veranschaulichung wurden Säulendiagramme, Tortendiagramme und Boxplots angefertigt. Boxplots zeigen Median und Interquartilsabstand (25\% und $75 \%$ Quantil) an. Ausreißer bzw. Extremwerte werden durch Kreise bzw. Sterne dargestellt.

Kategoriale Zielvariablen wurden mittels Chi-Quadrat-Test (z.B. Blutung auf Sondierung) zwischen den Gruppen „Sofortimplantation“ und „Spätimplantation“ getestet.

Für die Auswertung der Sondierungstiefen, der PPI-Werte und des Knochenabbaus wurde abhängig vom Endpunkt eine parametrische oder nichtparametrische Kovarianzanalyse (ANOVA) angewendet und bei den Sondierugstiefen für einen späteren 
Paarvergleich mittels Bonferroni-Methode adjustiert. Als Einflussfaktoren werden der Implantationszeitpunkt, die Position und deren Wechselwirkung geprüft.

Für die ordinalen Zielvariablen aus dem Fragebogen wurde ein Mann-Whitney-U-Test benutzt, um Unterschiede abhängig vom Implantationszeitpunkt zu überprüfen.

Für alle statistischen Tests wird ein Signifikanzniveau von $\alpha=5 \%$ festgesetzt. Das bedeutet, dass ein p -Wert kleiner 0,05 auf eine Ablehnung der Nullhypothese hindeutet (Mittelwerts- oder Verteilungsgleichheit). 


\section{Resultate}

\subsection{Die Überlebensrate}

Im Rahmen der telefonischen Befragung konnte ein Implantatverlust festgestellt werden. Dieser Implantatverlust trat nach 2 Monaten innerhalb der Gruppe der Sofortimplantationen auf. Die Gesamtüberlebensrate in dieser Studie beträgt somit nach einem mittleren Beobachtungszeitraum von 46 Monaten $98 \%(\mathrm{n}=52)$. Die Überlebensrate der Implantate in der Gruppe der Spätimplantationen beträgt $100 \%$ ( $\mathrm{n}=19)$ und in der Gruppe der Sofortimplantationen 96,97\% ( $\mathrm{n}=33)$.

\subsection{Beurteilung der Patientenzufriedenheit}

Alle 44 Patienten machten Angaben zu den folgenden Punkten.

\subsubsection{Beurteilung des Gesamtresultates der Behandlung}

$59 \%$ der befragten Patienten bewerteten das Gesamtresultat der Behandlung als „,sehr gut“, $39 \%$ als „gut“ und $2 \%$ als „befriedigend“ (siehe Abb. 3.1). Eine Übersicht über die vergebenen Schulnoten bietet die Tabelle 3.1.

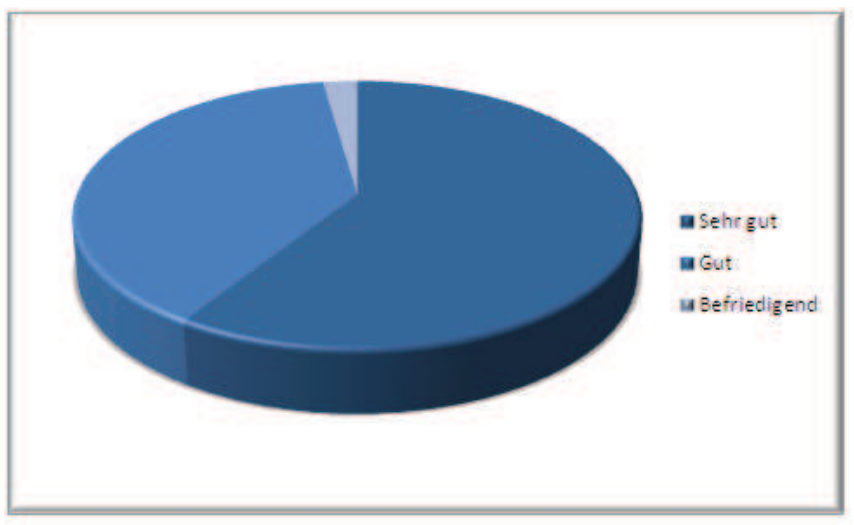

Abbildung 3.1: Diagramm Beurteilung des Gesamtresultates der Behandlung 


\begin{tabular}{lcccc}
\hline & N & $\begin{array}{r}\text { Sehr } \\
\text { gut }\end{array}$ & Gut & Befriedigend \\
\hline Sofortimpl. & 31 & 19 & 11 & 1 \\
Spätimpl. & 13 & 7 & 6 & 0 \\
Gesamt & 44 & 26 & 17 & 1 \\
\hline
\end{tabular}

Tabelle 3.1: Beurteilung des Gesamtresultates der Behandlung (deutsche Schulnote)

Bei der Beurteilung des Gesamtresultates der Behandlung mittels der visuellen Analogskalen konnte ein Mittelwert von 90,56 mm bestimmt werden, wobei $100 \mathrm{~mm}$ dem bestmöglichen Behandlungsergebnis entsprachen (siehe Tabelle 3.2).

\begin{tabular}{lcccccc}
\hline & N & MW & Median & Min. & Max. & Std.abw. \\
\hline Sofortimpl. & 31 & 90,34 & 95,00 & 50,00 & 100,00 & 13,04 \\
Spätimpl. & 13 & 92,68 & 90,00 & 86,00 & 100,00 & 4,77 \\
Gesamt & 44 & 90,56 & 94,00 & 50,00 & 100,00 & 11,34 \\
\hline
\end{tabular}

Tabelle 3.2: Beurteilung des Gesamtresultates (visuelle Analogskala (mm))

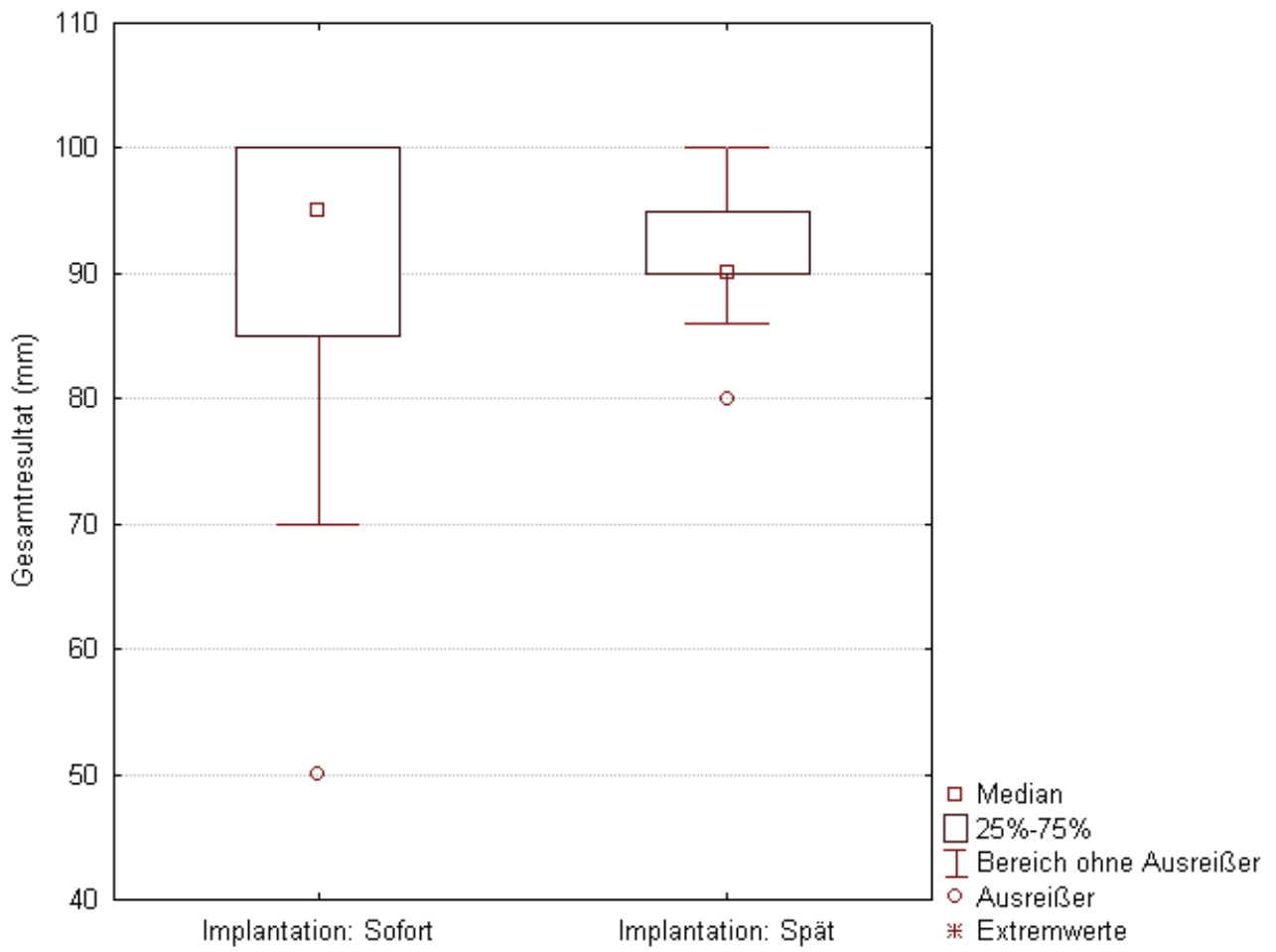

Abbildung 3.2: Boxplot Gesamtresultat der Behandlung

Die Abb. 3.2 veranschaulicht die Auswertung der visuellen Analogskalen. Nur ein Patient aus der Gruppe der Sofortimplantationen gab auf der Skala einen Wert unter 70 
mm an. Es wurde im Mann-Whitney-U-Test ein p-Wert von 0,66 ermittelt. Bezüglich des Implantationszeitpunktes konnte daher kein signifikanter Unterschied der Zufriedenheit der Patienten mit dem Gesamtresultat der Behandlung festgestellt werden ( $p$ $>0,05)$.

\subsubsection{Bewertung der Ästhetik}

Auf die Frage, wie sie das Aussehen ihrer implantologischen Versorgung bewerten, antworteten $59 \%$ der Patienten ,sehr gut“ und $41 \%$ der Patienten „gut“ (siehe Abb. 3.3). Kein Patient bewertete die Ästhetik des Zahnersatzes mit einer Note schlechter als „gut" (siehe Tab. 3.3).

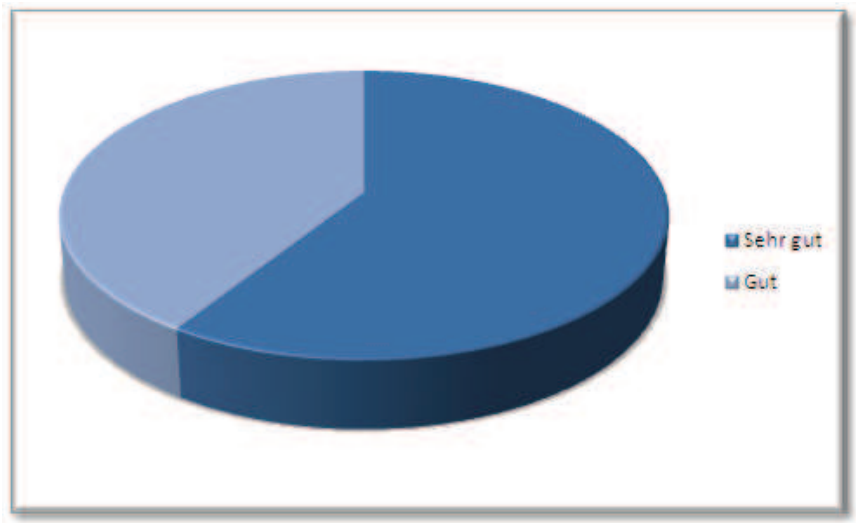

Abbildung 3.3: Diagramm Bewertung der Ästhetik

\begin{tabular}{lcccc}
\hline & N & $\begin{array}{c}\text { Sehr } \\
\text { gut }\end{array}$ & Gut & Befriedigend \\
\hline Sofortimpl. & 31 & 15 & 16 & 0 \\
Spätimpl. & 13 & 11 & 2 & 0 \\
Gesamt & 44 & 26 & 18 & 0 \\
\hline
\end{tabular}

Tabelle 3.3: Bewertung der Ästhetik (deutsche Schulnote)

Bei der Auswertung der visuellen Analogskalen konnte ein Mittelwert von 90,17 mm bestimmt werden. Eine Übersicht über die ermittelten Daten bietet die Tabelle 3.4.

\begin{tabular}{lcccccc}
\hline & N & MW & Median & Min. & Max. & Std.abw. \\
\hline Sofortimpl. & 31 & 89,15 & 90,00 & 50,00 & 100,00 & 11,51 \\
Spätimpl. & 13 & 93,37 & 95,00 & 80,00 & 100,00 & 6,49 \\
Gesamt & 44 & 90,17 & 92,00 & 50,00 & 100,00 & 10,39 \\
\hline
\end{tabular}

Tabelle 3.4: Bewertung der Ästhetik (visuelle Analogskala (mm)) 
Das Boxplot Diagramm in der Abb. 3.4 lässt erkennen, dass es nur in der Gruppe der Sofortimplantate Bewertungen unter $80 \mathrm{~mm}$ gab. Im Mann-Whitney-U-Test konnte bei der Bewertung kein statistisch signifikanter Unterschied zwischen den Patienten mit Sofort- und denen mit Spätimplantaten festgestellt werden ( $\mathrm{p}$-Wert von 0,33 ( $\mathrm{p}>$ $0,05))$.

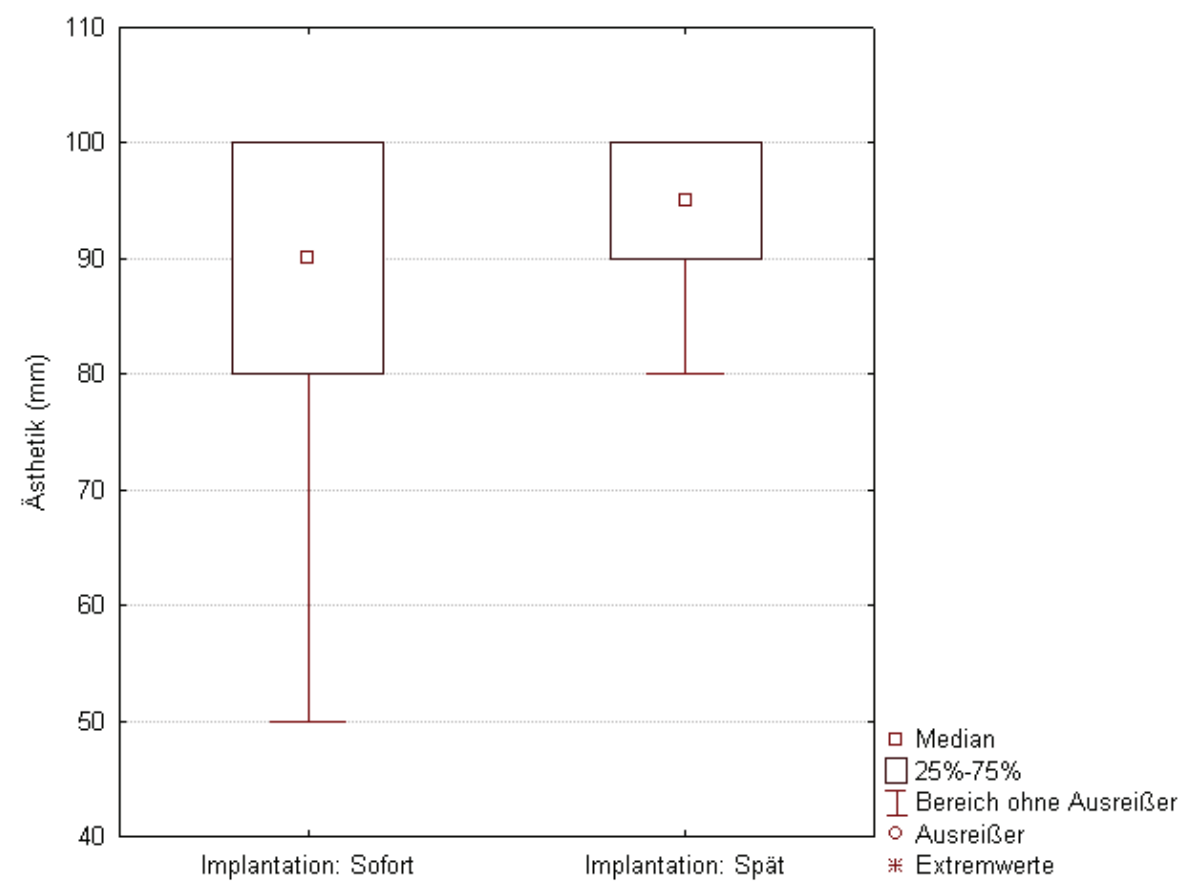

Abbildung 3.4: Boxplot Bewertung der Ästhetik

\subsubsection{Bewertung der Abbeißfunktion}

Die Abbeißfunktion ihrer implantologischen Versorgung beurteilten $52 \%$ der Patienten als ,sehr gut“, $41 \%$ als „gut" und $7 \%$ als „befriedigend“ (siehe Abb. 3.5). Kein Patient aus der Gruppe der Spätimplantationen vergab eine Note schlechter als „gut“ (siehe Tab. 3.5).

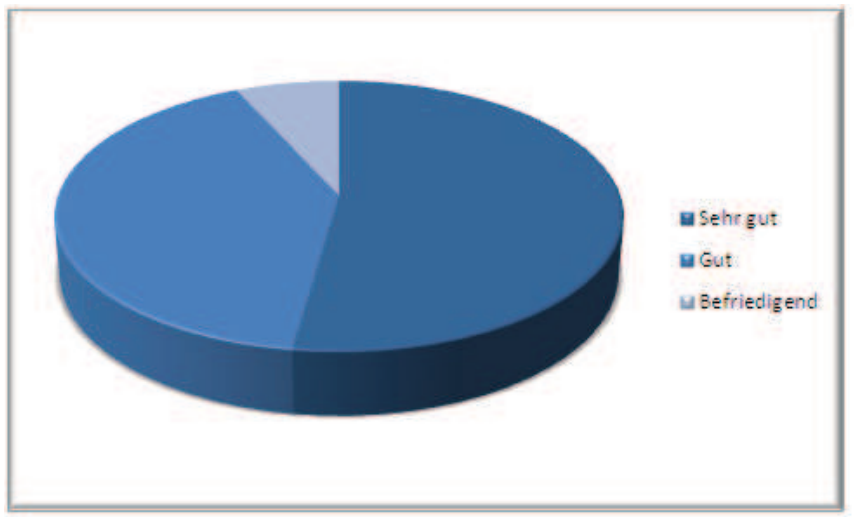

Abbildung 3.5: Diagramm Bewertung der Abbeißfunktion 


\begin{tabular}{lcccc}
\hline & $\mathrm{N}$ & $\begin{array}{r}\text { Sehr } \\
\text { gut }\end{array}$ & Gut & Befriedigend \\
\hline Sofortimpl. & 31 & 15 & 13 & 3 \\
Spätimpl. & 13 & 8 & 5 & 0 \\
Gesamt & 44 & 23 & 18 & 3 \\
\hline
\end{tabular}

Tabelle 3.5: Bewertung Abbeiffunktion (deutsche Schulnote)

Die Tabelle 3.6 gibt einen Überblick über die Daten der Auswertung der visuellen Analogskalen. Ein Mittelwert von 89,70 mm konnte anhand der visuellen Analogskalen bestimmt werden.

\begin{tabular}{lcccccc}
\hline & N & MW & Median & Min. & Max. & Std.abw. \\
\hline Sofortimpl. & 31 & 87,97 & 90,00 & 50,00 & 100,00 & 12,09 \\
Spätimpl. & 13 & 93,85 & 95,00 & 80,00 & 100,00 & 6,48 \\
Gesamt & 44 & 89,70 & 90,50 & 50,00 & 100,00 & 11,00 \\
\hline
\end{tabular}

Tabelle 3.6: Bewertung der Abbeißfunktion (visuelle Analogskala (mm))

Das Boxplot Diagramm in der Abb. 3.6 lässt erkennen, dass es in der Gruppe der Sofortimplantate schlechtere Bewertungen der Abbeißfunktion gab, als in der Gruppe der Spätimplantate. Trotzdem war keine statistische Signifikanz beim Vergleich der Zufriedenheit mit der Abbeißfunktion zwischen den Patienten mit Spät- und Sofortimplantaten feststellbar ( $\mathrm{p}$-Wert von $0,13(\mathrm{p}>0,05)$.

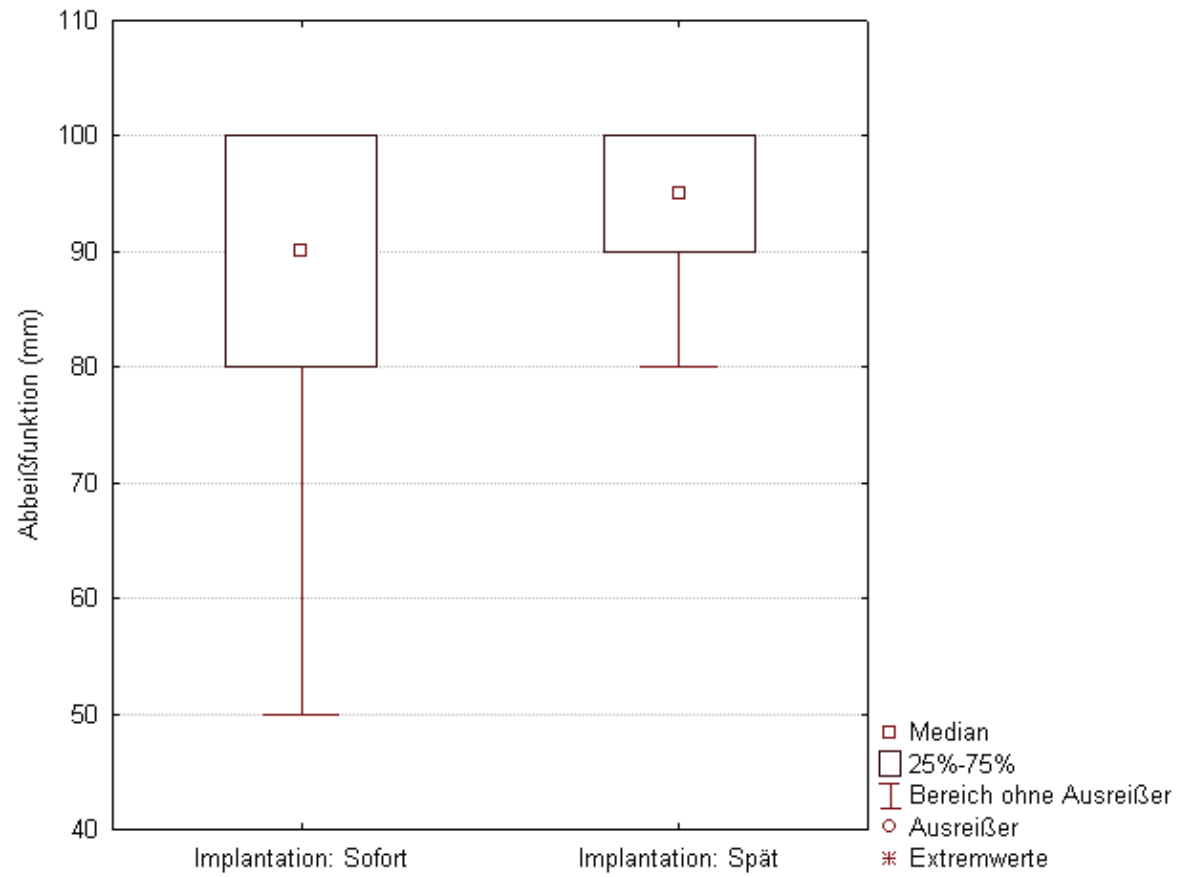

Abbildung 3.6: Boxplot Bewertung der Abbeißfunktion 


\subsubsection{Bewertung der Phonetik}

$80 \%$ der Patienten betrachten ihre Sprachfunktion als ,sehr gut", $11 \%$ als ,gut“ und $9 \%$ als „befriedigend“ (siehe Abb. 3.7) .

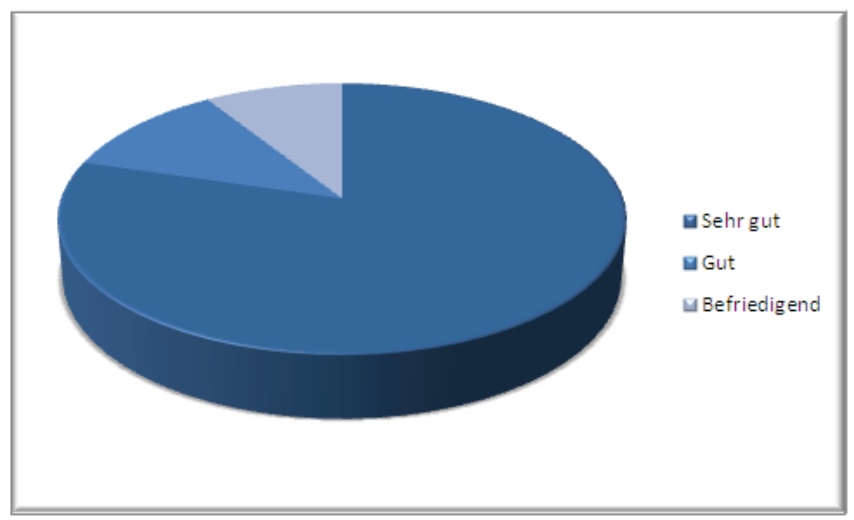

Abbildung 3.7: Diagramm Bewertung der Phonetik

Im Gegensatz zu den Patienten mit Sofortimplantaten vergab kein Patient aus der Gruppe der Spätimplantate eine Note schlechter als „sehr gut“ (siehe Tab. 3.7).

\begin{tabular}{lcccc}
\hline & N & $\begin{array}{r}\text { Sehr } \\
\text { gut }\end{array}$ & Gut & Befriedigend \\
\hline Sofortimpl. & 31 & 22 & 5 & 4 \\
Spätimpl. & 13 & 13 & 0 & 0 \\
Gesamt & 44 & 35 & 5 & 4 \\
\hline
\end{tabular}

Tabelle 3.7: Bewertung Sprachfunktion (deutsche Schulnote)

Eine Zusammenfassung der ermittelten Daten der visuellen Analogskalen bietet die Tabelle 3.8. 92,57 $\mathrm{mm}$ von den best möglichen $100 \mathrm{~mm}$ betrug die durchschnittliche Zufriedenheit der Patienten mit ihrer Sprachfunktion.

\begin{tabular}{lcccccc}
\hline & N & MW & Median & Min. & Max. & Std.abw. \\
\hline Sofortimpl. & 31 & 90,18 & 99,00 & 50,00 & 100,00 & 15,88 \\
Spätimpl. & 13 & 98,22 & 100,00 & 94,00 & 100,00 & 2,44 \\
Gesamt & 44 & 92,57 & 99,00 & 50,00 & 100,00 & 13,83 \\
\hline
\end{tabular}

Tabelle 3.8: Bewertung der Sprachfunktion (visuelle Analogskala (mm)) 


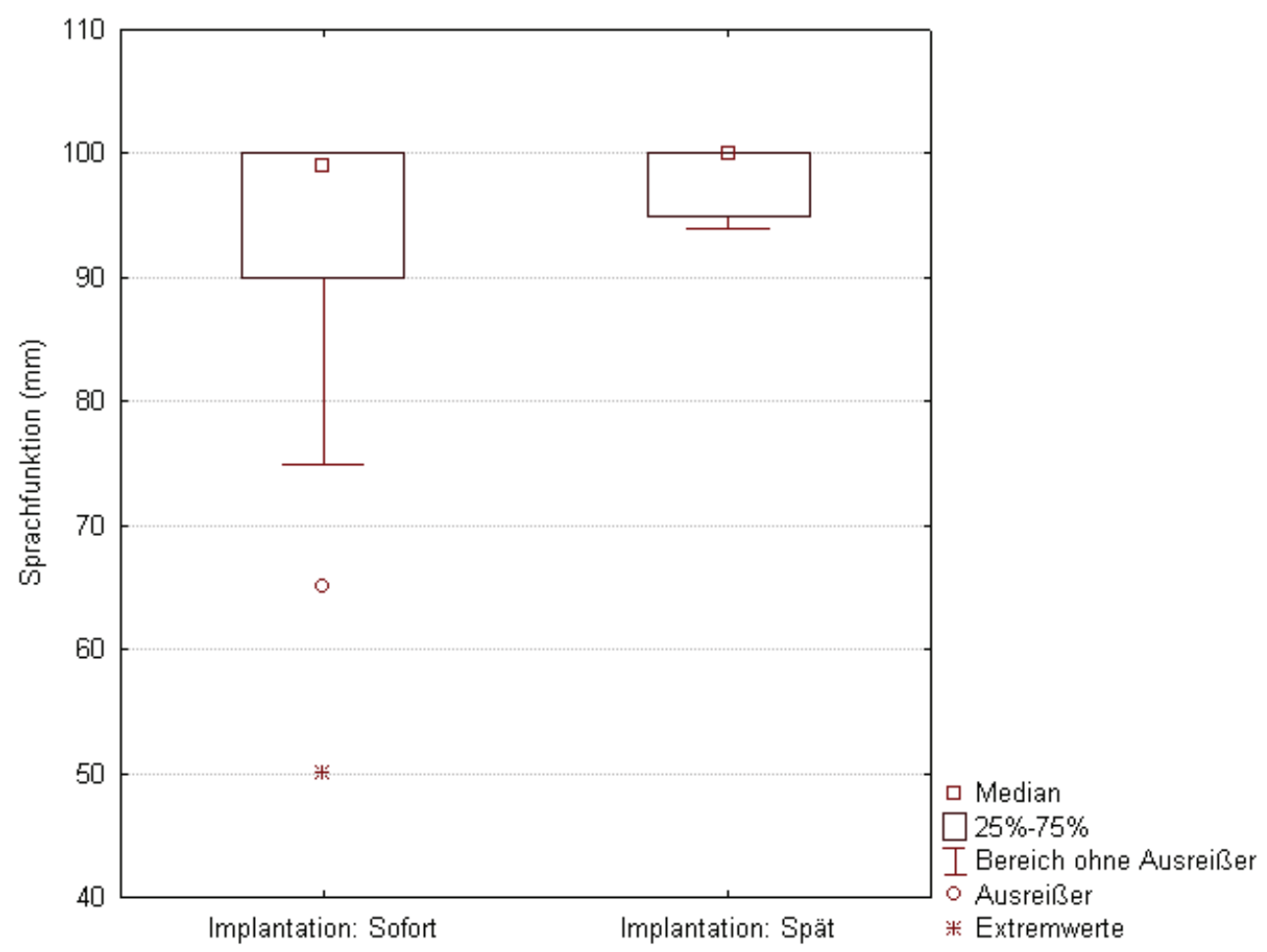

Abbildung 3.8: Boxplot Beurteilung der Sprachfunktion

Betrachtet man die Abb. 3.8, fällt auf, dass es bei den Sofortimplantate 2 Ausreißer im Sinne einer schlechteren Bewertung der Phonetik gab. Obwohl kein Patient aus der Gruppe der Spätimplantate eine schlechtere Note als ,sehr gut“ vergab (siehe Tab. 3.7), konnte im Mann-Whitney-U-Test zwischen den Bewertungen der Gruppen der Sofortund Spätimplantate keine statistische Signifikanz festgestellt werden (p- Wert von 0,23 $(\mathrm{p}>0,05))$.

\subsubsection{Bewertung der Hygienefähigkeit der Implantate}

Die Hygienefähigkeit ihrer implantologischen Versorgung bewerteten $73 \%$ der Patienten als ,sehr gut“, $20 \%$ als „gut“ und $7 \%$ als „befriedigend“ (siehe Abb. 3.9).

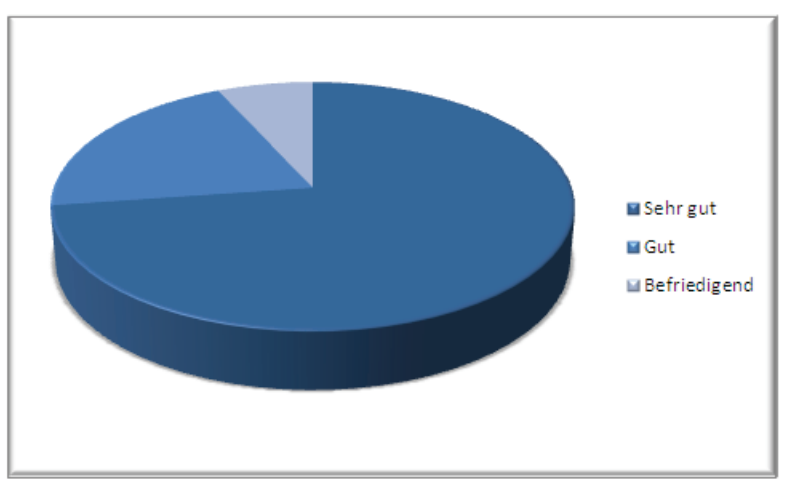

Abbildung 3.9: Diagramm Bewertung der Hygienefähigkeit 
Es wurde keine Note schlechter als „befriedigend“ vergeben (siehe Tab. 3.9). In Bezug auf die Hygienefähigkeit der Sofort- und Spätimplantate konnte kein statistisch relevanter Unterschied im Mann-Whitney-U-Test festgestellt werden (p-Wert von 0,13 (p $>0,05)$ ).

\begin{tabular}{lcccc}
\hline & N & $\begin{array}{c}\text { Sehr } \\
\text { gut }\end{array}$ & Gut & Befriedigend \\
\hline Sofortimpl. & 31 & 24 & 6 & 1 \\
Spätimpl. & 13 & 8 & 3 & 2 \\
Gesamt & 44 & 32 & 9 & 3 \\
\hline
\end{tabular}

Tabelle 3.9: Bewertung der Hygienefähigkeit (deutsche Schulnote)

\subsubsection{Anzahl der Zahnarztbesuche aufgrund von Komplikationen}

Im mittleren Beobachtungszeitraum von 46 Monaten mussten die Patienten durchschnittlich 1 mal aufgrund von Beschwerden mit ihren Implantaten einen Zahnarzt aufsuchen (siehe Tabelle 3.10).

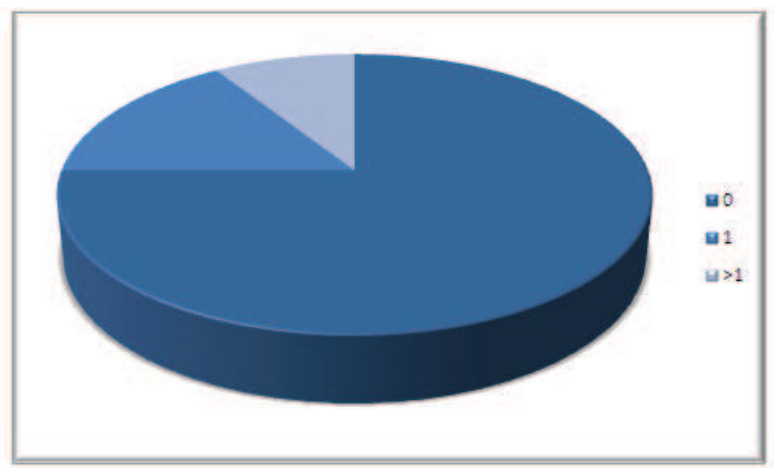

Abbildung 3.10: Diagramm Anzahl der Zahnarztbesuche aufgrund von Beschwerden

\begin{tabular}{lcccccc}
\hline & N & MW & Median & Min. & Max. & Std.abw. \\
\hline Sofortimpl. & 31 & 1,06 & 0 & 0 & 20 & 3,66 \\
Spätimpl. & 13 & 0,13 & 0 & 0 & 1 & 0,35 \\
Gesamt & 44 & 0,76 & 0 & 0 & 20 & 3,03 \\
\hline
\end{tabular}

Tabelle 3.10: Anzahl der Zahnarztbesuche aufgrund von Beschwerden

Der Medianwert (Median 0) lässt erkennen, dass der Großteil der Patienten keine behandlungsbedürftigen Beschwerden zu beklagen hatte. Insgesamt hatten $75 \%$ der Pa- 
tienten keinerlei Beschwerden und nur $9 \%$ der Patienten mussten öfter als 1- mal einen Zahnarzt konsultieren (siehe Abb. 3.10).

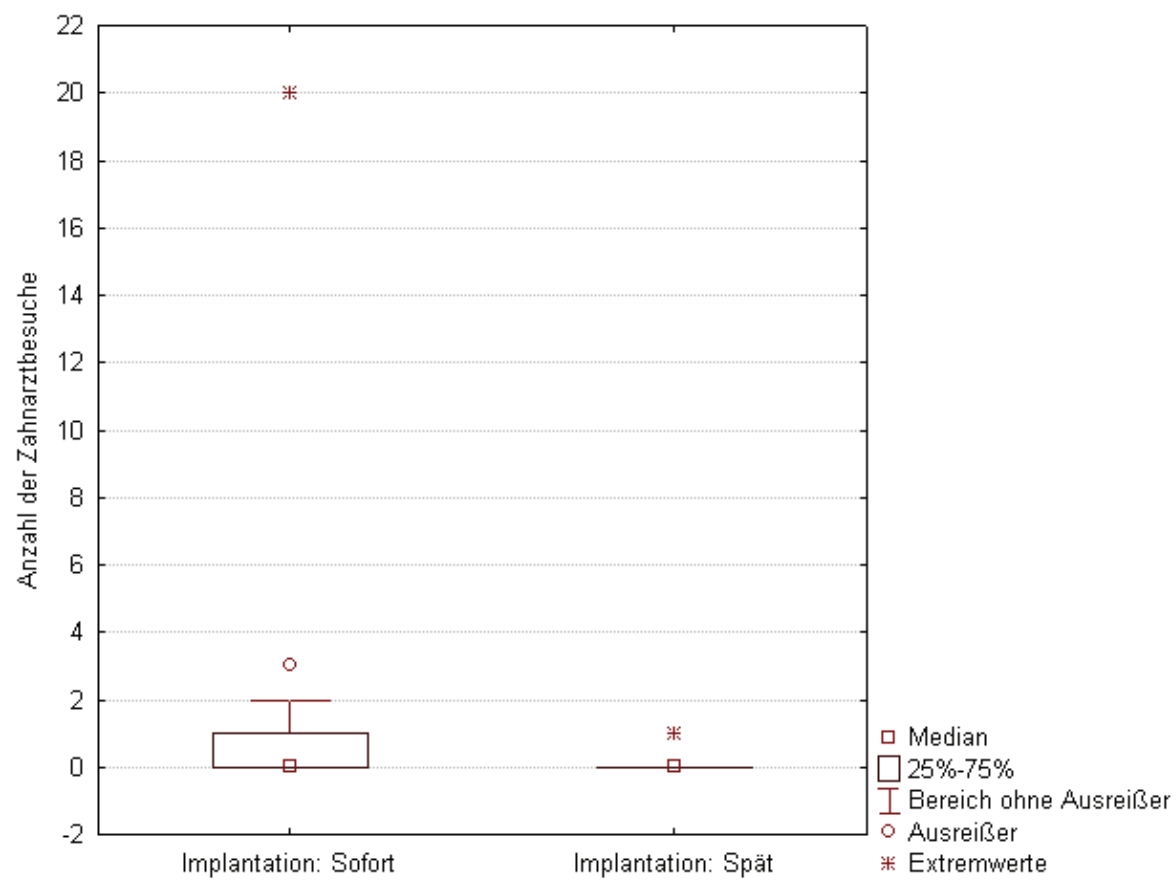

Abbildung 3.11: Boxplot Anzahl der Zahnarztbesuche aufgrund von Beschwerden

Das Boxplot Diagramm in der Abb. 3.11 lässt erkennen, dass ein Patient in der Gruppe der Sofortimplantate angab aufgrund von Beschwerden 20 mal den Zahnarzt aufgesucht zu haben. Trotz alledem konnte im Mann-Whitney-U-Test kein signifikanter Unterschiede in Bezug auf den Implantationszeitpunkt festgestellt werden (p-Wert von $0,28(\mathrm{p}>0,05))$.

\subsubsection{Nachfrage zur Behandlungsempfehlung}

$95 \%$ der befragten würden die Implantation mit kortikaler Mikrofixation einem guten Bekannten weiterempfehlen (siehe Abb. 3.12).

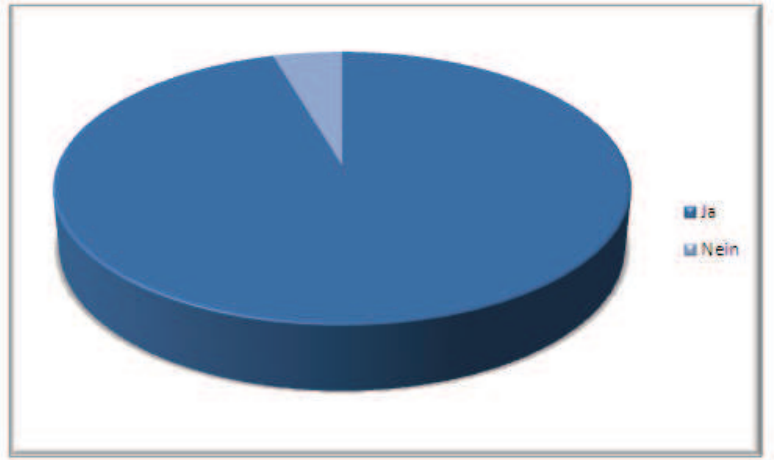

Abbildung 3.12: Diagramm Behandlungsempfehlung 
Nur 2 Patienten (5\%) beantworteten diese Frage verneinend (siehe Tab. 3.11).

\begin{tabular}{lccc}
\hline & N & Ja & Nein \\
\hline Sofortimpl. & 31 & 29 & 2 \\
Spätimpl. & 13 & 13 & 0 \\
Gesamt & 44 & 42 & 2 \\
\hline
\end{tabular}

Tabelle 3.11: Behandlungsempfehlung (Ja/Nein)

Die Tabelle 3.12 fasst die Daten der Auswertung der visuellen Analogskalen zusammen. Ein Mittelwert von 90,73 mm konnte bestimmt werden.

\begin{tabular}{lcccccc}
\hline & N & MW & Median & Min. & Max. & Std.abw. \\
\hline Sofortimpl. & 31 & 90,15 & 98,00 & 0,00 & 100,00 & 24,40 \\
Spätimpl. & 13 & 92,08 & 99,00 & 50,00 & 100,00 & 14,69 \\
Gesamt & 44 & 90,73 & 98,50 & 0,00 & 100,00 & 21,83 \\
\hline
\end{tabular}

Tabelle 3.12: Behandlungsempfehlung (visuelle Analogskala (mm))

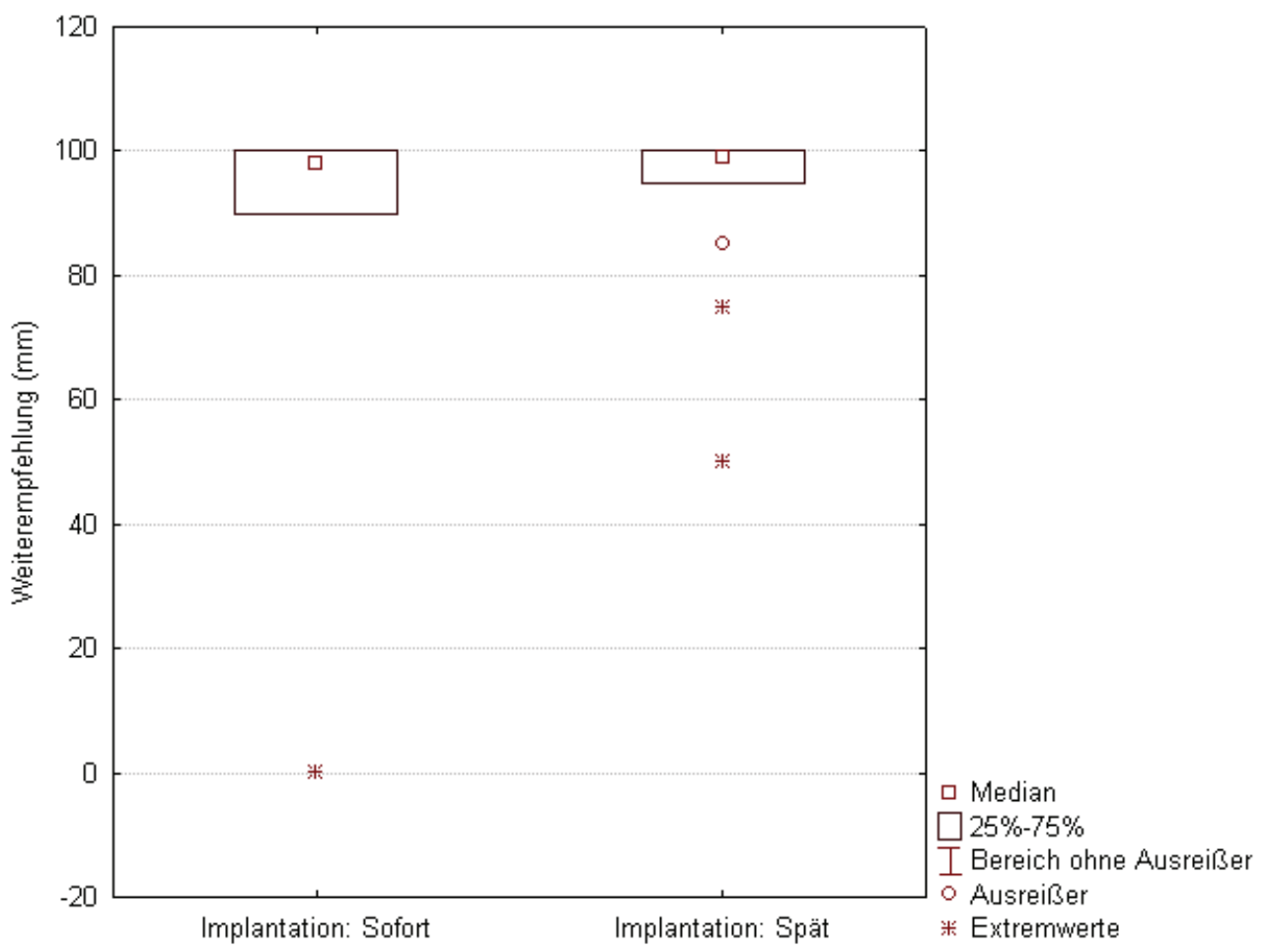

Abbildung 3.13: Boxplot Behandlungsempfehlung 
Betrachtet man die Abb. 3.13 ist nur ein Extremwert unter $50 \mathrm{~mm}$ in der Gruppe der Sofortimplantate zu erkennen. Dies bedeutet, dass der Großteil der Patienten sehr wahrscheinlich eine Weiterempfehlung der Satellitenimplantation aussprechen würde. Es gab keinen statistisch signifikanten Unterschied bei den Antworten der Patienten mit Sofort- und Spätimplantaten ( $\mathrm{p}$-Wert von $0,71(\mathrm{p}>0,05))$.

\subsubsection{Nachfrage zur erneuten Behandlungswilligkeit}

Auf die Frage, ob Sie sich erneut einer Satellitenimplantation unterziehen würden, antworteten $98 \%$ der Befragten mit „Ja“ (siehe Abb. 3.14).

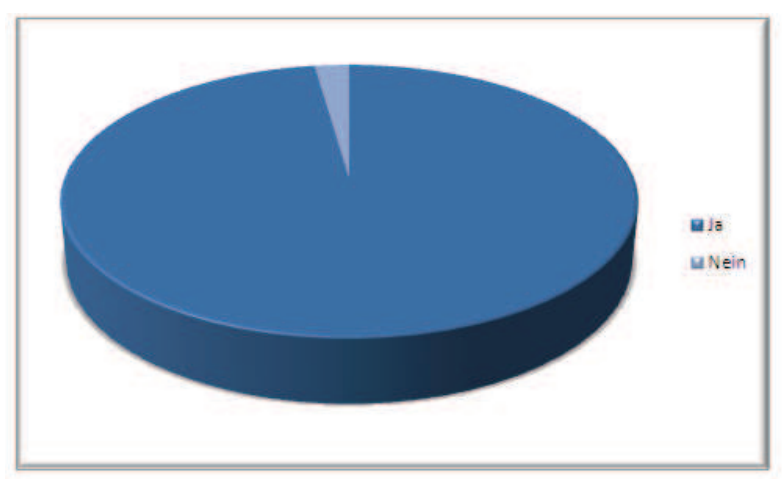

Abbildung 3.14: Diagramm erneute Behandlungswilligkeit

Lediglich einer (2 \%) der befragten Patienten würde eine erneute Behandlung ablehnen (siehe Tab. 3.13).

\begin{tabular}{lccc}
\hline & N & Ja & Nein \\
\hline Sofortimpl. & 31 & 30 & 1 \\
Spätimpl. & 13 & 13 & 0 \\
Gesamt & 44 & 43 & 1 \\
\hline
\end{tabular}

Tabelle 3.13: Erneute Behandlungswilligkeit (Ja/Nein)

Die Daten der Auswertung der visuellen Analogskalen in Tabelle 3.14 sowie in der Abb. 3.15 lassen erkennen, dass sich die meisten Patienten sehr wahrscheinlich erneut einer Implantation mit kortikaler Mikrofixation unterziehen würden (Mittelwert 92,08 mm). Nur 2 Patienten kreuzten auf der Skala einen Wert unter $60 \mathrm{~mm}$ an. Zwischen der erneuten Behandlungswilligkeit der Patienten mit Sofort- und Spätimplantaten konnte kein signifikanter Unterschied im Mann-Whitney-U-Test festgestellt werden (p- Wert von $0,98(\mathrm{p}>0,05))$. 


\begin{tabular}{lcccccc}
\hline & N & MW & Median & Min. & Max. & Std.abw. \\
\hline Sofortimpl. & 31 & 91,90 & 99,00 & 0,00 & 100,00 & 18,73 \\
Spätimpl. & 13 & 92,54 & 99,00 & 50,00 & 100,00 & 14,00 \\
Gesamt & 44 & 92,08 & 99,00 & 0,00 & 100,00 & 17,30 \\
\hline
\end{tabular}

Tabelle 3.14: Erneute Behandlungwilligkeit (visuelle Analogskala (mm))

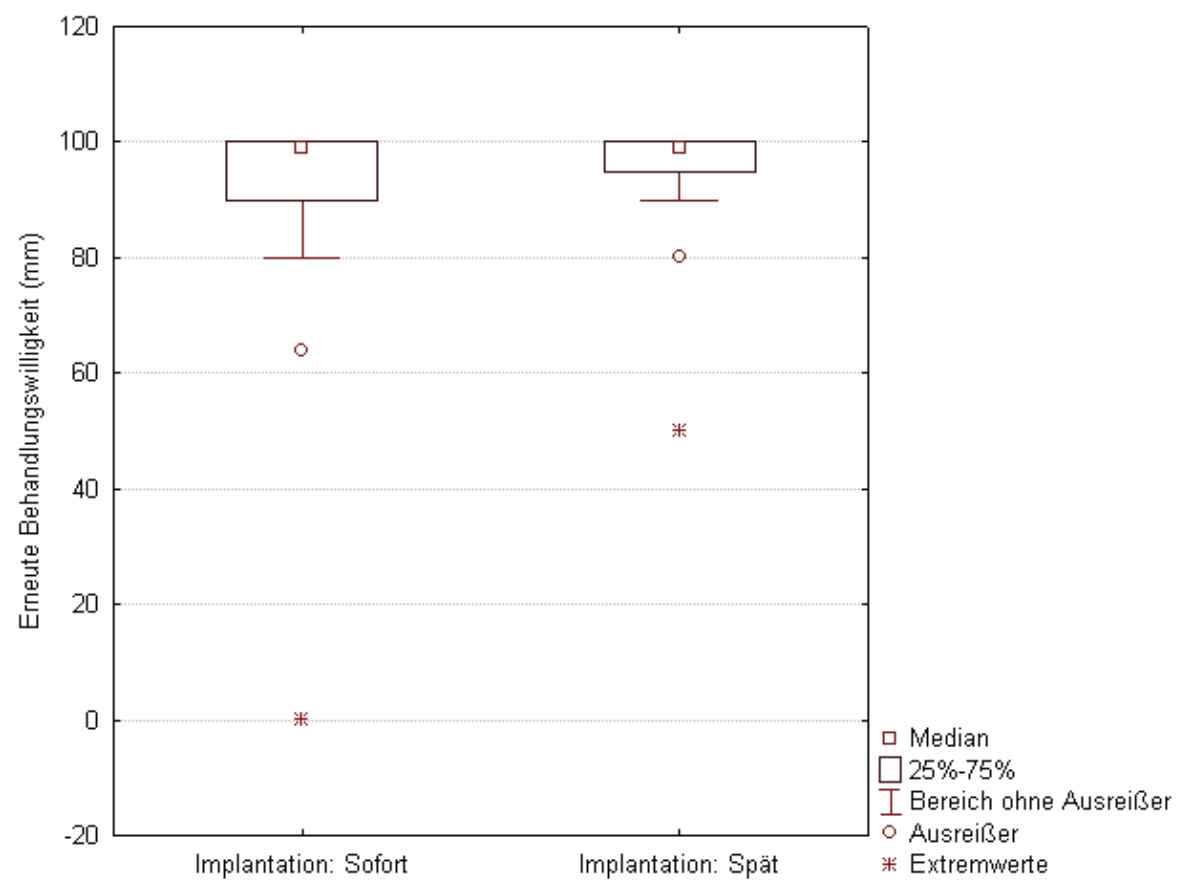

Abbildung 3.15: Boxplot Erneute Behandlungswilligkeit

\subsubsection{Nachfrage zur Zufriedenheit mit der Mundgesundheit}

Im Rahmen der Befragung gaben 10 von 44 Patienten an (23\%), nicht mit ihrer Mundgesundheit zufrieden zu sein (siehe Abb. 3.16). Diese Patienten stammten alle aus der Gruppe der Sofortimplantationen (siehe Tabelle 3.15).

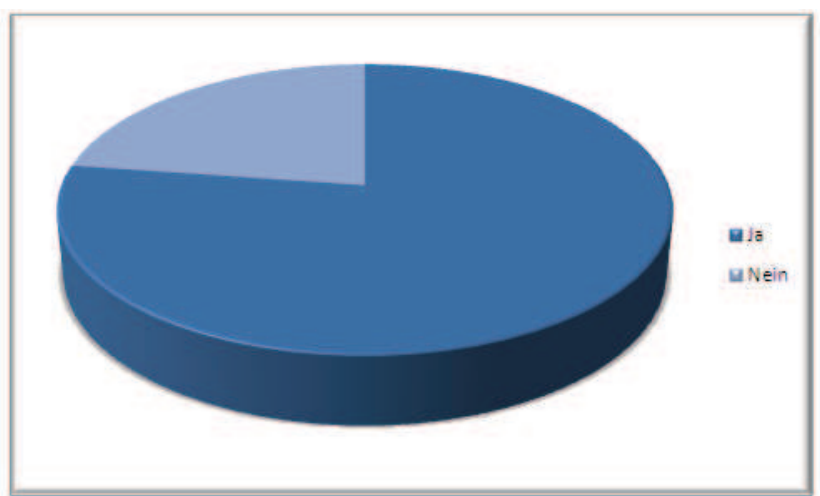

Abbildung 3.16: Diagramm Zufriedenheit mit Mundgesundheit 


\begin{tabular}{lccc}
\hline & $\mathrm{N}$ & $\mathrm{Ja}$ & Nein \\
\hline Sofortimpl. & 31 & 21 & 10 \\
Spätimpl. & 13 & 13 & 0 \\
Gesamt & 44 & 34 & 10 \\
\hline
\end{tabular}

Tabelle 3.15: Zufriedenheit mit Mundgesundheit (Ja/Nein)

Die Tabelle 3.16 präsentiert die Datenauswertung der visuellen Analogskalen und wird in der Abb. 3.17 nochmals veranschaulicht. Man kann erkennen, dass die Patientengruppe der Sofortimplantate im Boxplot Diagramm eine wesentlich größere Streuung der Werte im Sinne einer schlechteren Beurteilung der Mundgesundheit zeigt. Der Mittelwert betrug 81,14 mm. Im Mann-Whitney-U-Test konnte kein statistisch signifikanter Unterschied zwischen den Patienten mit Sofort- und Spätimplantaten in Bezug auf die Zufriedenheit mit der Mundgesundheit festgestellt werden ( $\mathrm{p}$-Wert von 0,22 ( $\mathrm{p}>$ $0,05))$.

\begin{tabular}{lcccccc}
\hline & N & MW & Median & Min. & Max. & Std.abw. \\
\hline Sofortimpl. & 31 & 75,84 & 80,00 & 30,00 & 100,00 & 24,06 \\
Spätimpl. & 13 & 89,14 & 80,00 & 60,00 & 100,00 & 11,74 \\
Gesamt & 44 & 81,14 & 89,50 & 30,00 & 100,00 & 18,42 \\
\hline
\end{tabular}

Tabelle 3.16: Zufriedenheit mit Mundgesundheit (visuelle Analogskala (mm))

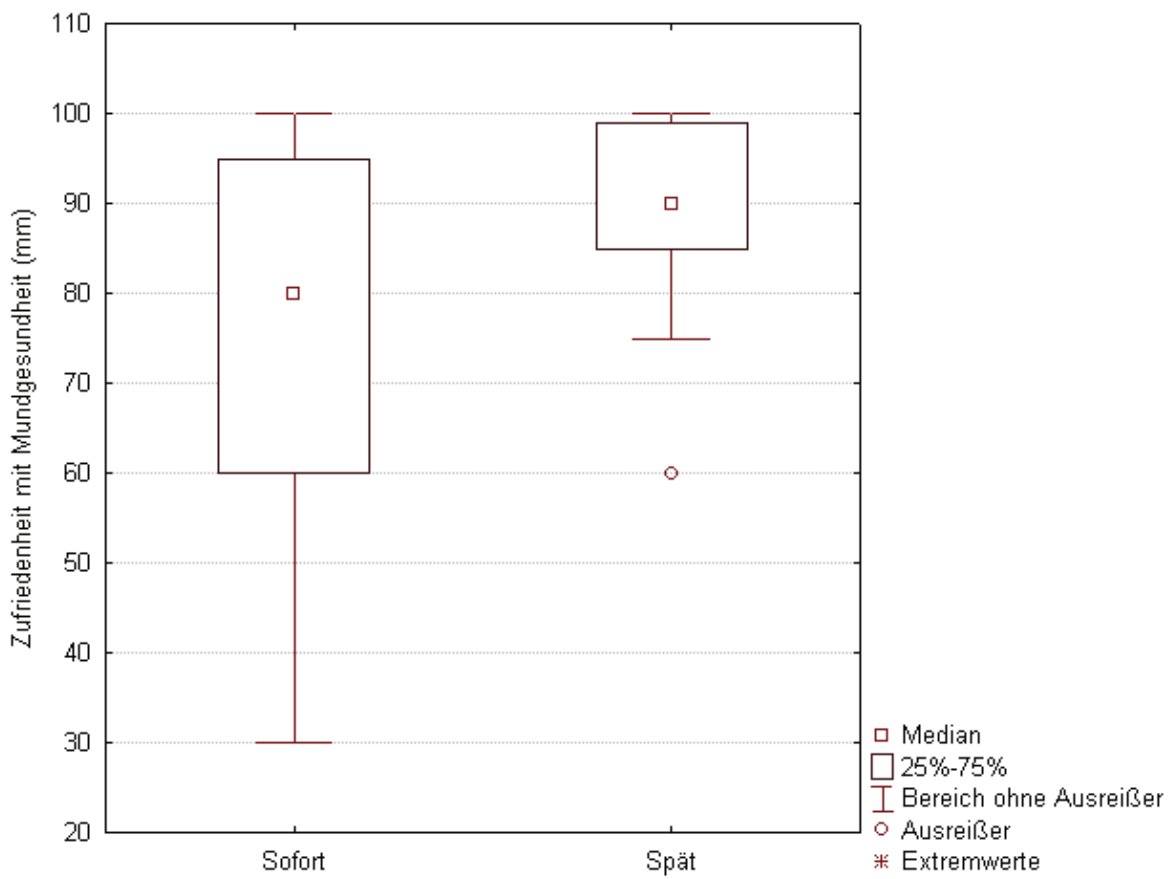

Abbildung 3.17: Boxplot Zufriedenheit mit Mundgesundheit 


\subsection{Die Risikofaktoren}

Die Tabelle 3.17 präsentiert die an den 40 Implantaten $(n=40)$ röntgenologisch erhobenen Risikofaktoren (siehe S. 28), deren Einfluss auf das Implantationsergebnis im Rahmen der Kovarianzanalyse der Sondierungstiefen, des PPI-Indices und des Knochenabbaus geprüft wurden.

\begin{tabular}{lc}
\hline Risikofaktoren & Häufigkeit \\
\hline Abstand der SZG zum Limbus alveolaris am NZ > 3 mm & 5 \\
Anzahl der Implantate mit benachbarten Wurzelkanalfüllungen & 12 \\
Anzahl der Implantate mit einem erweiterten PA-Spalt am NZ & 3 \\
Anzahl der Implantate mit einem apikalen Herd am NZ & 5 \\
\hline
\end{tabular}

Tabelle 3.17: Risikofaktoren

\subsection{Ergebnisse der Sondierungstiefen}

Die Ergebnisse der Datenanalyse der Messung der periimplantären Sondierungstiefen werden in der Tabelle 3.18 präsentiert. Es wurden an 40 Implantaten jeweils 4 Sondierungstiefen (mesial, vestibulär, palatinal, distal) erhoben.

\begin{tabular}{lllllll}
\hline & & $\mathrm{N}$ & $\mathrm{MW}$ & Median & Min. & Max. \\
\hline mesial & Sofortimpl. & 24 & 2,17 & 2,00 & 1,00 & 4,00 \\
& Spätimpl. & 16 & 2,37 & 2,00 & 1,00 & 4,00 \\
& Gesamt & 40 & 2,25 & 2,00 & 1,00 & 4,00 \\
\hline \multirow{2}{*}{ vestibulär } & Sofortimpl. & 24 & 1,83 & 2,00 & 1,00 & 3,00 \\
& Spätimpl. & 16 & 1,80 & 2,00 & 1,00 & 3,00 \\
& Gesamt & 40 & 1,80 & 2,00 & 1,00 & 3,00 \\
\hline \multirow{2}{*}{ palatinal } & Sofortimpl. & 24 & 1,78 & 1,50 & 1,00 & 6,00 \\
& Spätimpl. & 16 & 1,62 & 1,50 & 1,00 & 3,00 \\
& Gesamt & 40 & 1,72 & 1,50 & 1,00 & 6,00 \\
\hline \multirow{2}{*}{ distal } & Sofortimpl. & 24 & 2,28 & 2,00 & 1,00 & 5,00 \\
& Spätimpl. & 16 & 2,31 & 3,00 & 1,00 & 3,00 \\
& Gesamt & 40 & 2,30 & 2,00 & 1,00 & 5,00 \\
\hline
\end{tabular}

Tabelle 3.18: Sondierungstiefen $(\mathrm{mm})$

Der Mittelwert der jeweiligen Sondierungsposition betrug mesial 2,25 mm, vestibulär 1,80 mm, palatinal 1,72 mm und distal 2,30 mm. Im Boxplot-Diagramm (siehe Abb. 3.18) werden die Messergebnisse veranschaulicht. 

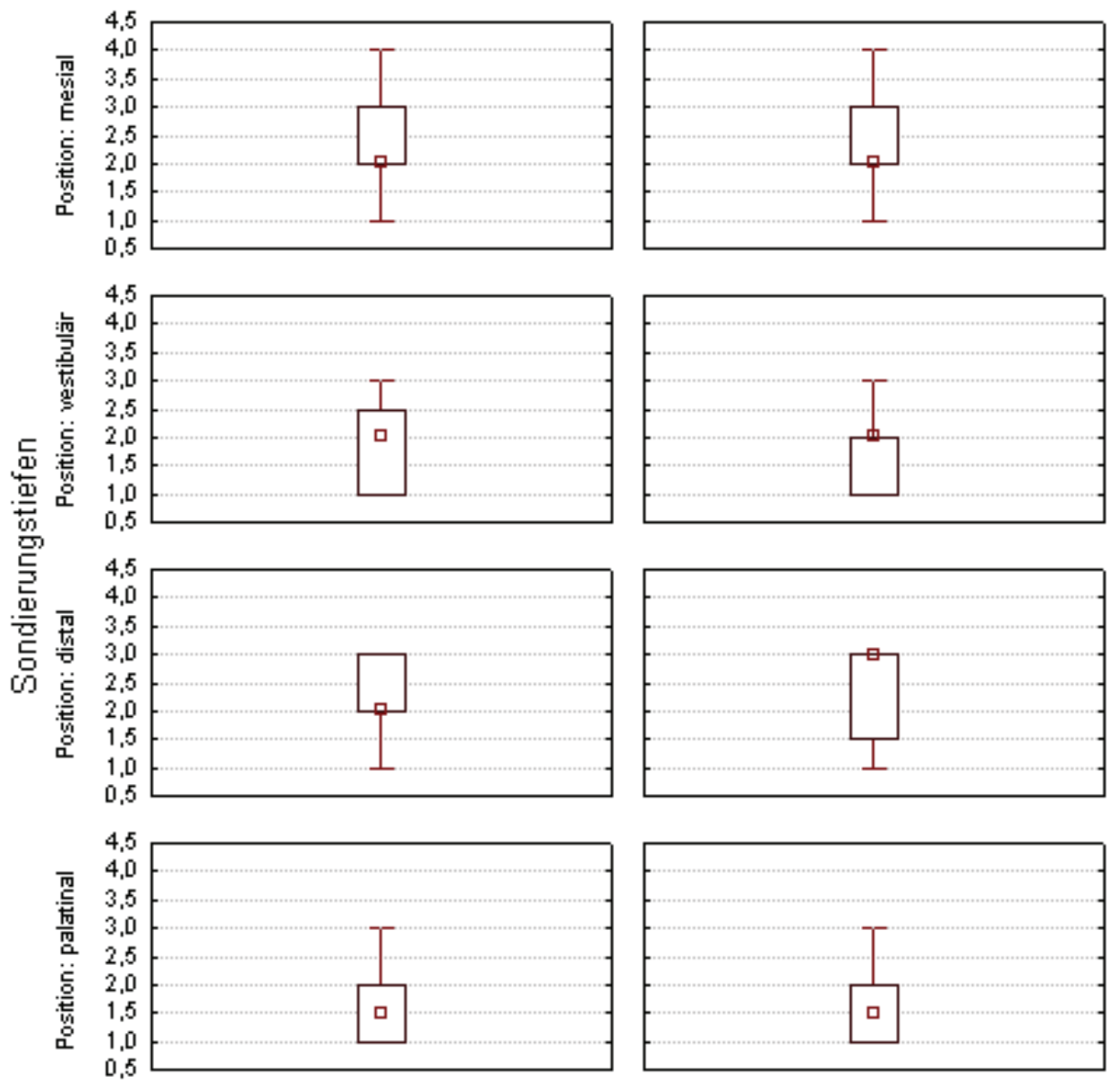

Implantation: Sofort

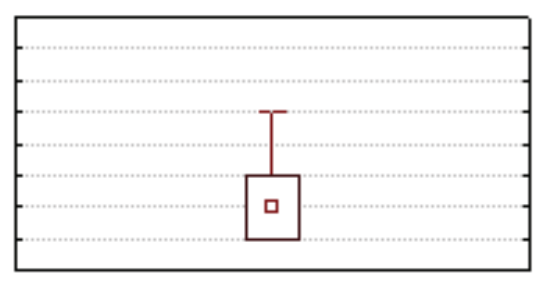

Implantation: Spät

$\square$ Median $\square 25 \%-75 \%$ I Bereich ohne Ausreißer

Abbildung 3.18: Boxplot Sondierungstiefen (mm)

Vergleicht man die Messergebnisse der einzelnen Sondierungspositionen sind bei den Sofort- und Spätimplantaten ähnliche Werte zu finden. Unabhängig von den Implantationszeitpunkt sind bei den approximalen Sondierungspositionen größere Taschentiefen gemessen wurden.

Die Darstellung der Konfidenzintervalle in den Abbildungen 3.19 und 3.20 lässt erkennen, dass sowohl bei den Spät- als auch bei den Sofortimplantaten das Konfidenzintervall der palatinalen Sondierung die geringsten Werte aufweist. 


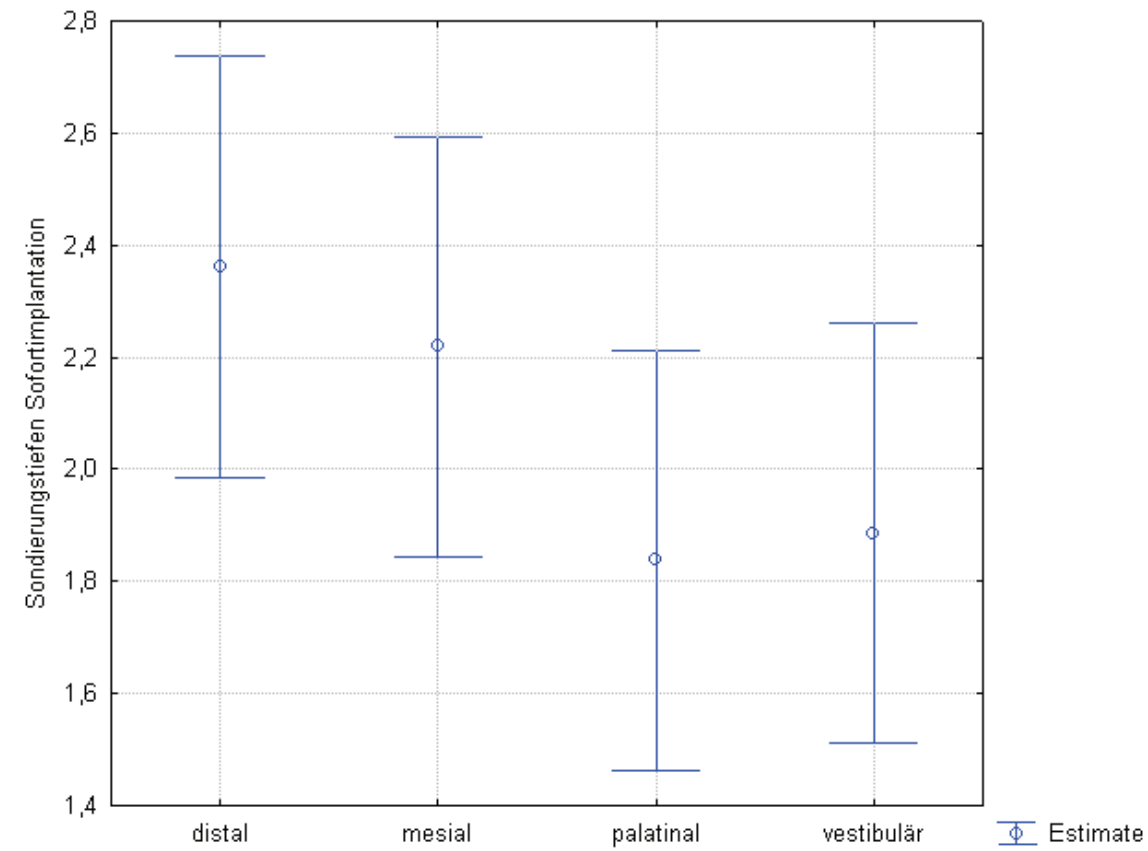

Abbildung 3.19: Intervallplot Sondierungstiefen (sofort $\mathrm{n}=24)(\mathrm{mm})$

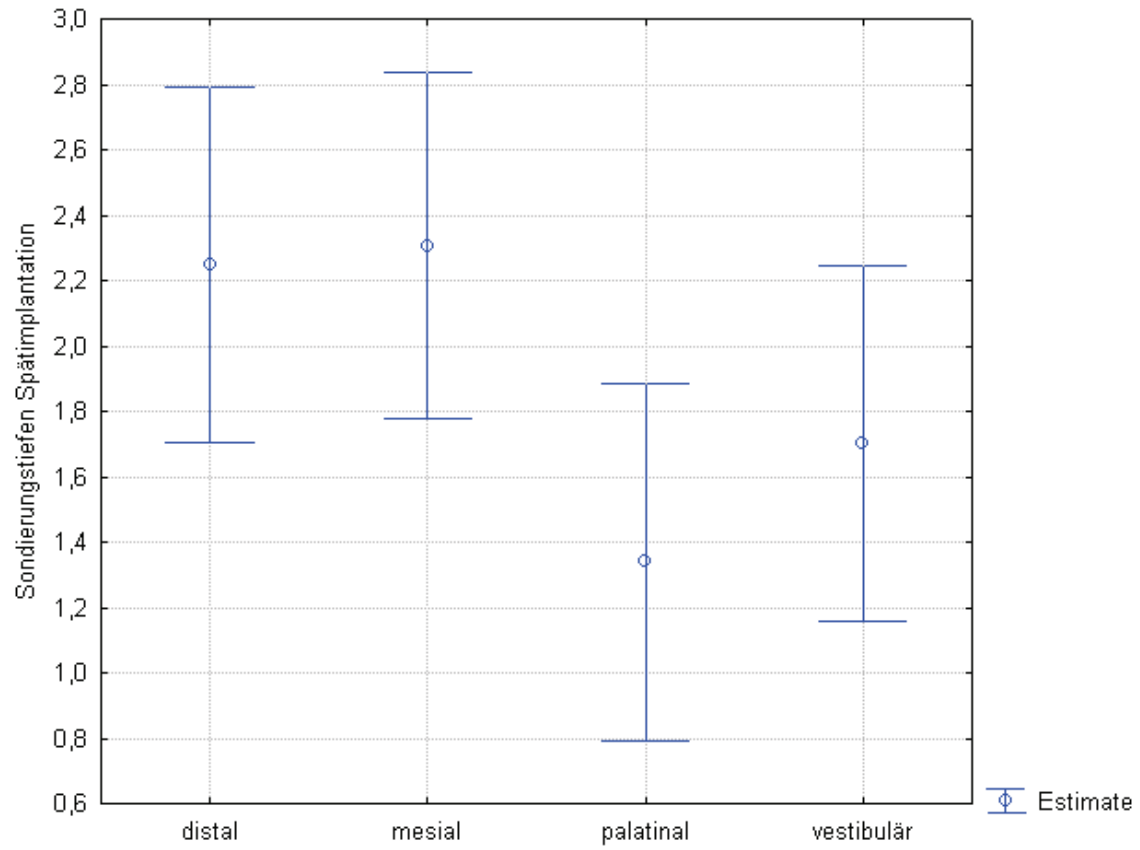

Abbildung 3.20: Intervallplot Sondierungstiefen (spät $\mathrm{n}=16)(\mathrm{mm})$

Als mögliche Einflussfaktoren wurden der Implantationszeitpunkt sowie die in Abschnitt 2.3.3 erwähnten lokalen Risikofaktoren auf ihre Signifikanz hin geprüft. Zusätzlich wurde auch der Einfluss der Position der Sondierung geprüft. Es konnte ein signifikanter Einfluss der Position der Sondierung (mesial/ vestibulär/ palatinal/ distal) auf die Sondierungstiefe beobachtet werden ( $\mathrm{p}$-Wert von 0,0001 ( $\mathrm{p}<0,05$ ). Darüber hinaus konnte das Vorhandensein eines erweiterten PA-Spaltes am Nachbarzahn 
als signifikanter Einflussfaktor identifiziert werden (p-Wert von 0,0135). Alle übrigen geprüften Kovariablen hatten keinen signifikanten Einfluss. Eine Übersicht über das Ergebnis der Kovarianzanalyse für die Sondierungstiefen bietet die Tabelle 3.19.

\begin{tabular}{lc}
\hline Einflussfaktor (Kovariable) & p-Wert \\
\hline Implantationszeitpunkt & 0,5591 \\
Position der Sondierung & 0,0001 \\
Abstand der Schmelzzementgrenze zum Limbus alveolaris am NZ & 0,3159 \\
Vorhandensein einer Wurzelkanalfüllung am NZ & 0,5906 \\
Vorhandensein eines erweiterten PA-Spaltes am NZ & 0,0135 \\
Vorhandensein eines apikalen Herdes am NZ & 0,4436 \\
\hline
\end{tabular}

Tabelle 3.19: Ergebnis der Kovarianzanalyse der Sondierungstiefen

Nach der Bonferroni-Adjustierung der Kovarianzanalyse (siehe Tab. 3.20) konnte festgestellt werden, dass die approximalen Sondierungspositionen untereinander (distal $/ \mathrm{mesial}$ ) keine signifikante Wechselwirkung aufweisen $(\mathrm{p}>0,05)$. Gleicherweise verhalten sich die palatinalen und vestibulären Sondierungspositionen zueinander $(p>0,05)$. Bei der Analyse der approximalen Sondierungspositionen im Verhältnis zu den vestibulären und palatinalen konnte jedoch ein signifikante Beziehung beobachtet werden $(\mathrm{p}<$ $0,05)$.

\begin{tabular}{lllc}
\hline & Position & Position & p-Wert \\
\hline Effekt & Distal & Mesial & 1,0000 \\
Effekt & Distal & Palatinal & 0,0003 \\
Effekt & Distal & Vestibulär & 0,0426 \\
Effekt & Mesial & Palatinal & 0,0003 \\
Effekt & Mesial & Vestibulär & 0,0493 \\
Effekt & Palatinal & Vestibulär & 0,7414 \\
\hline
\end{tabular}

Tabelle 3.20: Ergebnis der Bonferroni-Adjustierung der Kovarianzanalyse der Sondierungstiefen

\subsection{Blutungen auf Sondierung}

Beim Sondieren der periimplantären Taschen traten bei 10 von insgesamt 40 untersuchten Implantaten kleinere Blutungen auf. Dabei konnten bei 6 Sofortimplantaten (24 untersuchte Sofortimplantate) und 4 Spätimplantaten (16 untersuchte Spätimplantate) Blutungen festgestellt werden. Es traten also bei jeweils $25 \%$ der Sofort- und Spätimplantate Blutungen beim Sondieren auf (siehe Abb. 3.21). Bei der statistischen Analyse 
konnte kein signifikanter Einfluss des Implantationszeitpunktes auf das Auftreten von Blutungen festgestellt werden ( $\mathrm{p}$-Wert von 1).

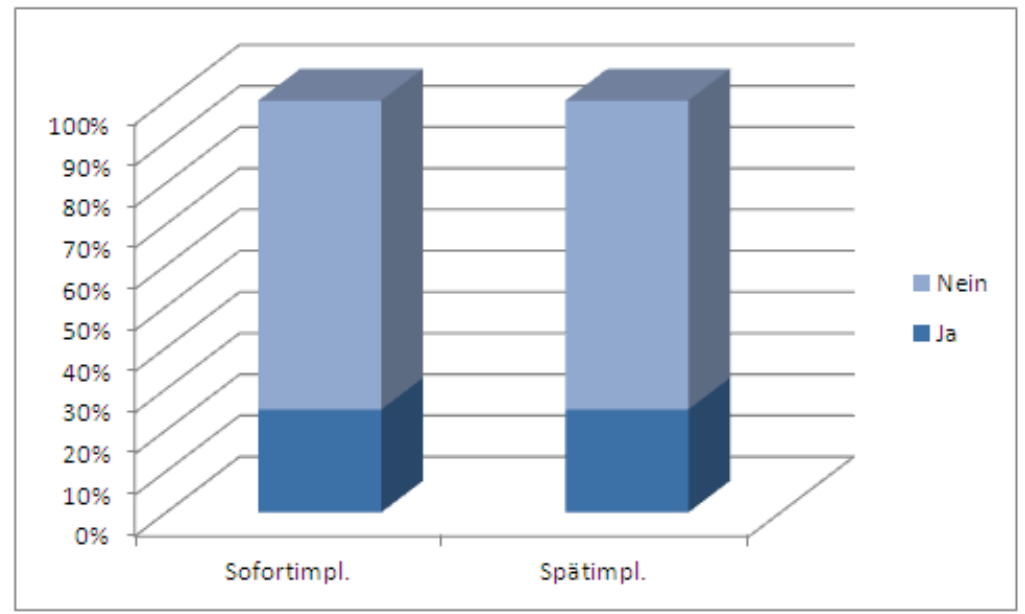

Abbildung 3.21: Säulendiagramm Blutung auf Sondierung (Ja/Nein)

\subsection{Papilla Presence Index}

Einen Überblick über die erhobenen PPI-Werte verschafft die Tabelle 3.21. Der Medianwert der Sofortimplantate betrug 2,00 und der Medianwert der Spätimplantate 1,5.

\begin{tabular}{llcccccc}
\hline & & N & Median & Min. & Max. & Q25 & Q75 \\
\hline PPI & Sofortimpl. & 24 & 2,00 & 1,00 & 3,00 & 2 & 2,50 \\
& Spätimpl. & 16 & 1,50 & 1,00 & 3,00 & 1,5 & 2,25 \\
& Gesamt & 40 & 2,00 & 1,00 & 3,00 & & \\
\hline
\end{tabular}

Tabelle 3.21: Übersicht PPI-Werte

Die Abb. 3.22 veranschaulicht die Verteilung aller PPI-Werte, während das Boxplot Diagramm in der Abb. 3.23 zwischen den Werten der Sofort- und Spätimplantate differenziert. Man kann erkennen, dass der Interquartilsabstand der Sofortimplantate höhere Werte aufweist als der Interquartilsabstand der Spätimplantate. 


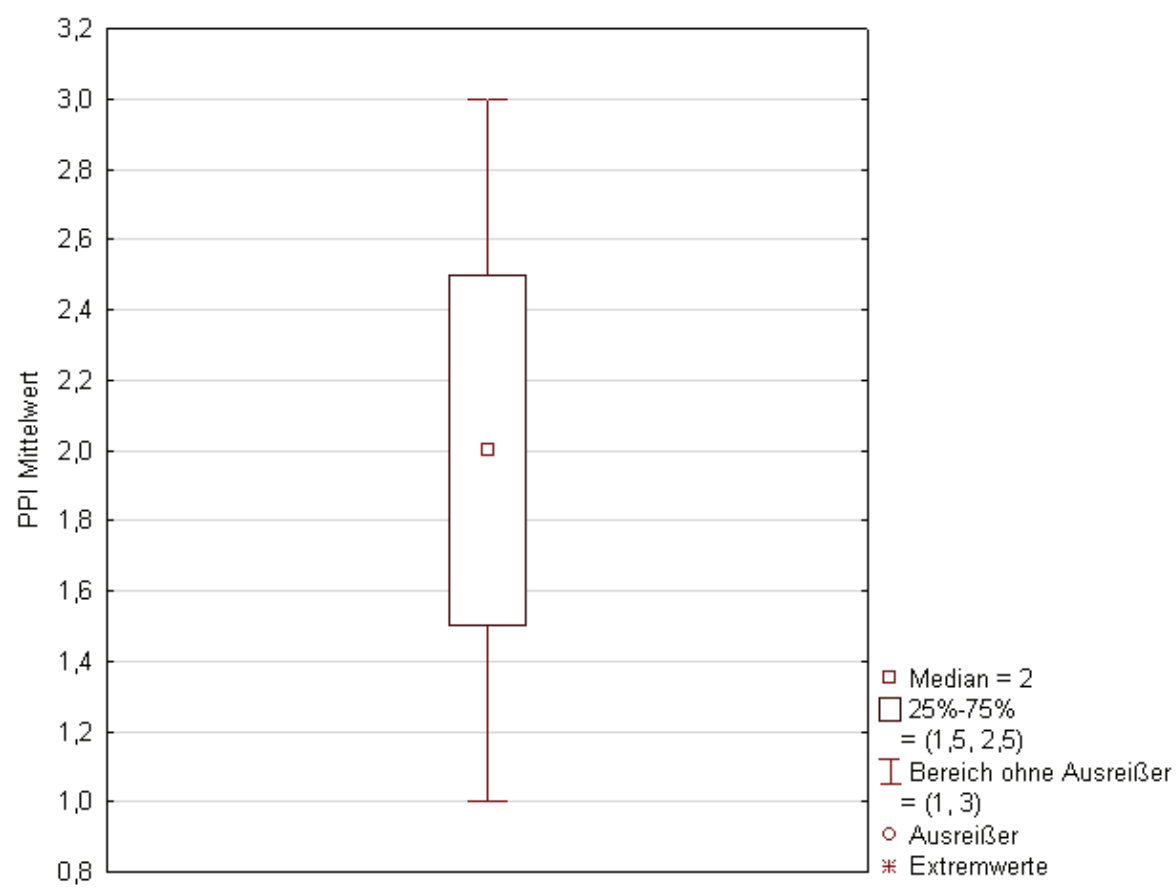

Abbildung 3.22: Boxplot PPI-Werte (gesamt)

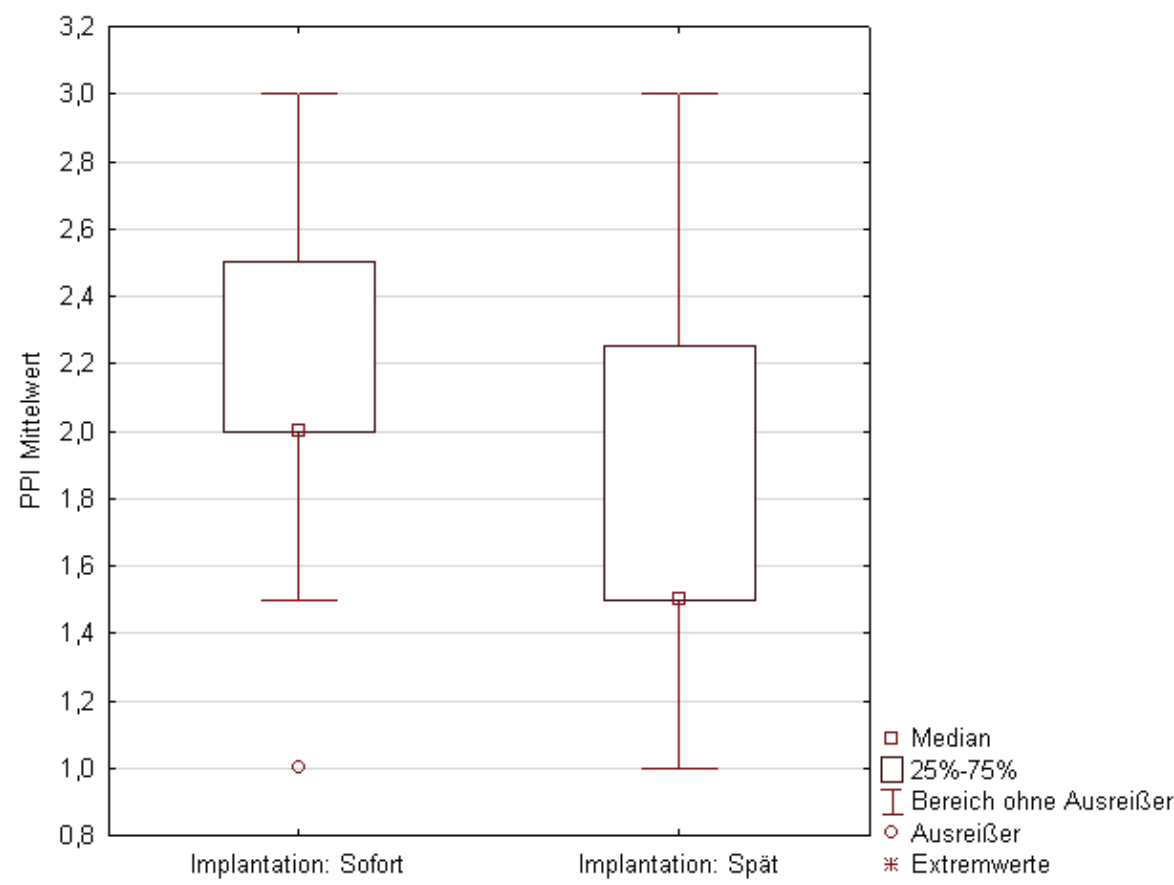

Abbildung 3.23: Boxplot PPI-Werte (sofort/spät)

Bei der nichtparametrischen Kovarianzanalyse der PPI-Werte konnte beobachtet werden, dass der Abstand der Schmelzzementgrenze vom Limbus Alveolaris an den Nachbarzähnen einen signifikanten Einfluss auf den PPI-Wert hat (p von 0,0001((siehe Tab. $3.22)$ ). 


\begin{tabular}{lc}
\hline Einflussfaktor (Kovariable) & p-Wert \\
\hline Implantationszeitpunkt & 0,9878 \\
Abstand der Schmelzzementgrenze zum Limbus alveolaris am NZ & 0,0001 \\
Vorhandensein einer Wurzelkanalfüllung am NZ & 0,5771 \\
Vorhandensein eines erweiterten PA-Spaltes am NZ & 0,7939 \\
Vorhandensein eines apikalen Herdes am NZ & 0,8900 \\
\hline
\end{tabular}

Tabelle 3.22: Ergebnis der Kovarianzanalyse der PPI-Werte

\subsection{Ergebnisse der Periotestmessung}

Im Zuge der Nachuntersuchung wurden bei 40 Implantaten Periotestwerte bestimmt. In der Tabelle 3.23 wird eine Übersicht über die erhobenen Messwerte vorgestellt. Der Medianwert der Periotestmessung der Sofortimplantate betrug 0 und der Medianwert der Spätimplantate -0,05. Der Medianwert aller Implantate betrug -0,02. Die Werte wurden hinsichtlich des möglichen Einflusses des Implantationszeitpunktes geprüft. Dabei konnte kein signifikanter Unterschied zwischen den erhobenen Werten bei den Sofort- und Spätimplantaten festgestellt werden ( $\mathrm{p}$-Wert von 0,947 ( $\mathrm{p}>0,05))$.

\begin{tabular}{llcccccc}
\hline & & N & MW & Median & Min. & Max. & Std.abw. \\
\hline \multirow{2}{*}{ Periotest } & Sofortimpl. & 24 & 0,00 & 0,00 & $-5,00$ & 9,00 & 2,72 \\
& Spätimpl. & 16 & $-0,05$ & 0,00 & $-5,00$ & 5,00 & 2,81 \\
& Gesamt & 40 & $-0,02$ & 0,00 & $-5,00$ & 3,00 & 2,71 \\
\hline
\end{tabular}

Tabelle 3.23: Übersicht der Periotestwerte

\subsection{Ergebnisse der Bestimmung des Knochenabbaus / -gewinns}

Die Datenanalyse des horizontalen Knochenabbaus / -gewinns wird in der Tabelle 3.24 veranschaulicht.

Der Knochenabbau konnte an 40 Implantaten bestimmt werden $(\mathrm{n}=40)$. Der Mittelwert der mesialen und distalen Messwerte wurde gebildet und zur Analyse verwendet. 


\begin{tabular}{lcccccccc}
\hline & & & & & & & Konfidenz & Konfidenz \\
untere & Mobere \\
Grenze & Mrenze \\
\hline Sofortimpl. & 24 & 0,98 & 0,75 & $-0,9$ & 5,25 & 1,29 & 0,38 & 1,47 \\
Spätimpl. & 16 & 0,71 & 0,60 & $-0,1$ & 1,25 & 0,48 & 0,05 & 1,44 \\
Gesamt & 40 & 0,87 & 0,60 & $-0,9$ & 5,25 & 1,04 & & \\
\hline
\end{tabular}

Tabelle 3.24: Messdaten des horizontalen Knochenabbaus/-gewinns

Unabhängig vom Implantationszeitpunkt konnte ein mittlerer Knochenverlust von 0,87 mm und ein Medianwert von 0,6 mm ermittelt werden (siehe Abb. 3.24).

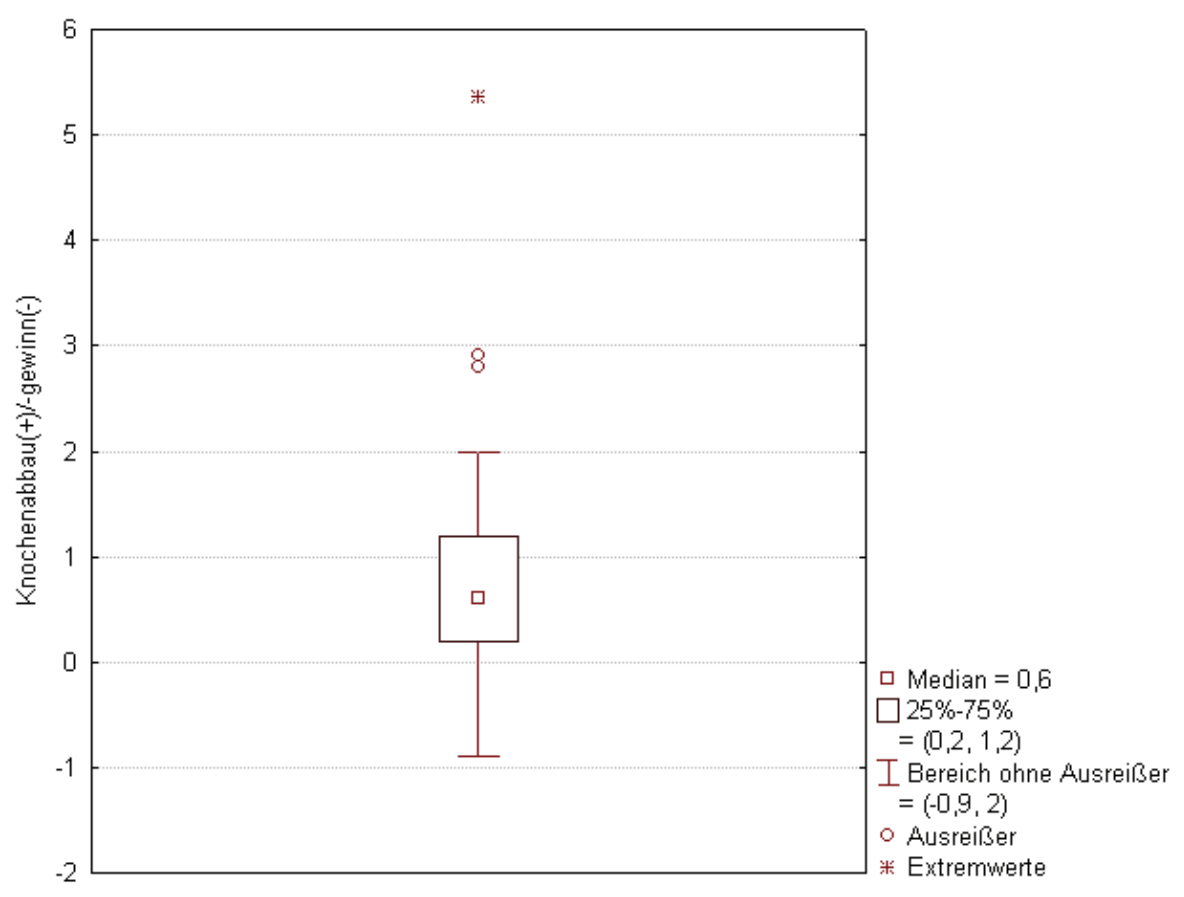

Abbildung 3.24: Boxplot Knochenabbau/-gewinn (gesamt)

Eine Differenzierung fand hinsichtlich der Sofort- und Spätimplantate statt (siehe Abb. $3.25)$.

Die Abb. 3.24 lässt erkennen, dass bei 3 Implantaten ein Knochenabbau von über 2 mm festgestellt werden konnte. Darunter waren 2 Sofortimplantate und 1 Spätimplantat (siehe Abb. 3.25). Ein maximaler Knochengewinn von 0,9 mm konnte bei einem Implantat aus der Gruppe der Sofortimplantationen beobachtet werden. Die ermittelten Konfidenzintervalle werden in der Abb. 3.26 veranschaulicht. 


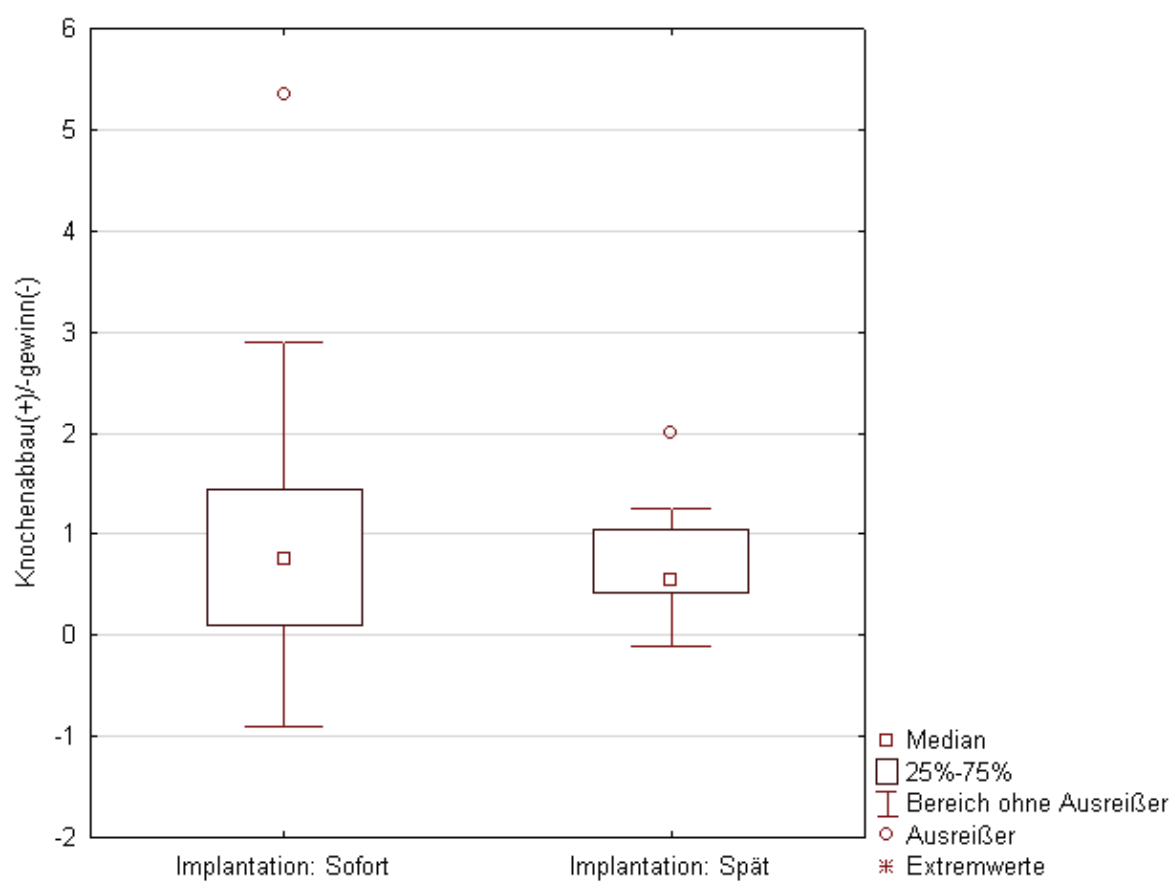

Abbildung 3.25: Boxplot Knochenabbau/-gewinn (sofort/spät)

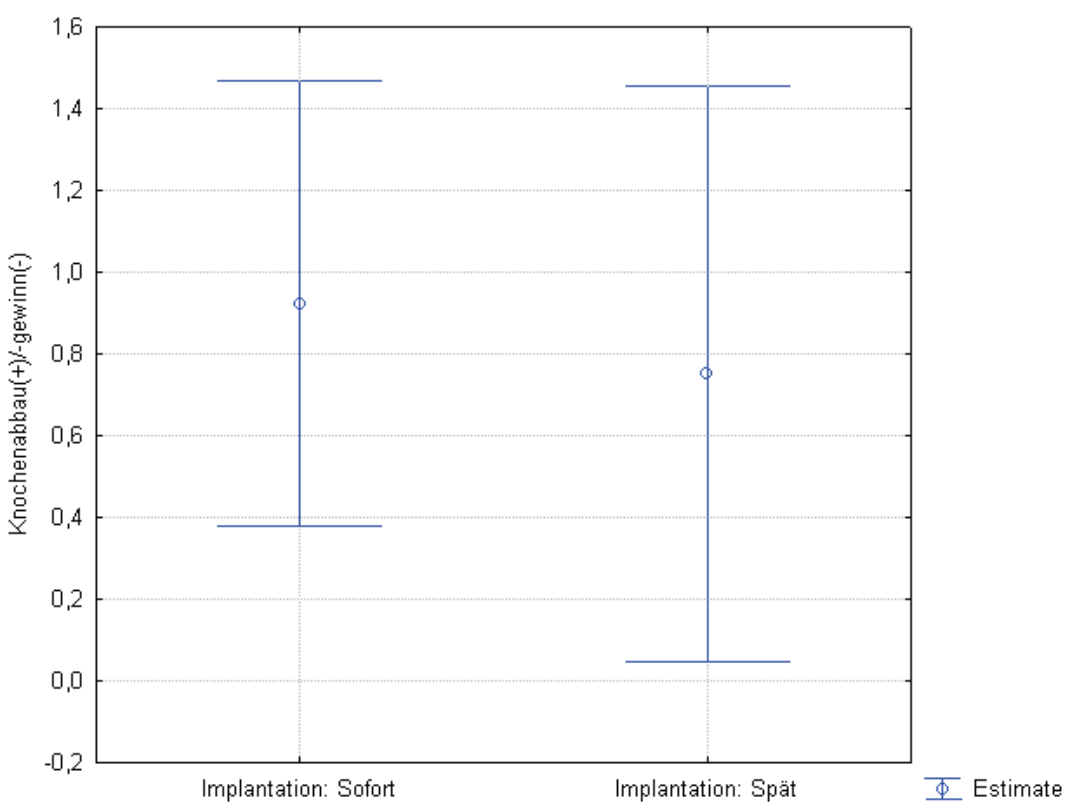

Abbildung 3.26: Intervallplot Knochenabbau/-gewinn

Vergleicht man die beiden Implantatgruppen, kann man feststellen, dass größere Werte die Grenzen der Konfidenzintervalle der Sofortimplantate bilden.

Bei der Kovarianzanalyse des horizontalen Knochenabbaus/- gewinns konnte, wie in Tabelle 3.25 dargestellt, nicht festgestellt werden, dass die Kovariablen einen signifikanten Einfluss auf den horizontalen Knochenabbau / -gewinn hatten ( $p>0,05)$. 


\begin{tabular}{lc}
\hline Einflussfaktor (Kovariable) & p-Wert \\
\hline Implantationszeitpunkt & 0,7018 \\
Abstand der Schmelzzementgrenze zum Limbus alveolaris am NZ & 0,6948 \\
Vorhandensein einer Wurzelkanalfüllung am NZ & 0,7971 \\
Vorhandensein eines erweiterten PA-Spaltes am NZ & 0,5007 \\
Vorhandensein eines apikalen Herdes am NZ & 0,3287 \\
\hline
\end{tabular}

Tabelle 3.25: Ergebnis der Kovarianzanalyse des Knochenabbaus/ -gewinns

\subsection{Die Erfolgsquote}

Bei 40 Implantaten fand eine klinische Nachuntersuchung mit radiologischer Bestimmung des Knochenabbaus statt sodass diese zur Beurteilung der Erfolgsquote herangezogen werden konnten $(\mathrm{n}=40)$. Als Misserfolg galt ein Implantat, sobald es ein Erfolgskriterium (siehe Tab. 2.4) nicht erfüllte, unabhängig von der Anzahl der nicht erfüllten Erfolgskriterien pro Implantat.

Betrachtet man die Gruppe der Patienten der klinischen Nachuntersuchung konnte ein Misserfolg in der Gruppe der Sofortimplantate festgestellt werden. Es handelte sich dabei um ein Implantat mit einer periimplantären Entzündung ausgehend von einem apikalen Herd am benachbarten Zahn. Am Implantat wurde ein Periotestwert von +9 und ein mittlerer röntgenologischer Knochenverlust von 5,35 mm gemessen. Trotz des Misserfolges bewertete die Patientin das Gesamtbehandlungsergebnis mit der Schulnote „befriedigend“. Daher ergab die Auswertung der Erfolgskriterien nach Jahn und d'Hoedt (1992) und Naert et al. (1992)/ Snawwaert et al. (2000) jeweils eine Erfolgsquote von $97,5 \%(\mathrm{n}=40)$.

In der Gruppe der telefonisch befragten Patienten war ein weiterer Implantatverlust in der Gruppe der Sofortimplantate zu vermerken. Würde man dieses Implantat in die Berechnung der Erfolgsquote mit einbeziehen (d.h. 2 Implantatmisserfolge), würde diese $95 \%(\mathrm{n}=41)$ betragen.

\subsection{Falldokumentationen}

In dieser Arbeit werden 2 Patientenfälle präsentiert, jeweils ein Spät- und ein Sofortimplantat. Beide Patienten wurden in der Praxis für zahnärztliche Chirurgie von Dr. Möller in Göttingen operiert.

Bei dem ersten Fall handelte es sich um eine 66- jährige Patientin, die im April 2006 operiert wurde. Infolge eines Zahnverlustes wurde ein Implantat in die Regio 022 inseriert. 
Bei dem Implantat handelte es sich um ein Standard Plus Implantat (Länge $14 \mathrm{~mm}$, Durchmesser 3,3 mm) der Firma Straumann. Das Implantat wurde durch zusätzliche palatinale Satellitenimplantate (Länge 7 und $5 \mathrm{~mm}$ ) stabilisiert, um eine Sofortversorgung des Implantates zu ermöglichen (siehe Abb. 3.27).Die Satellitenimplantate waren über eine 3-Loch-Osteosyntheseplatte mit dem Implantataufbauteil verbunden. Unmittelbar nach der Implantation wurde das Implantat mit einer provisorischen Krone versorgt. Nach einer erfolgreichen Implantateinheilung (6 Monate), konnten die Satellitenimplantate entfernt und eine definitive prothetische Versorgung eingegliedert werden.

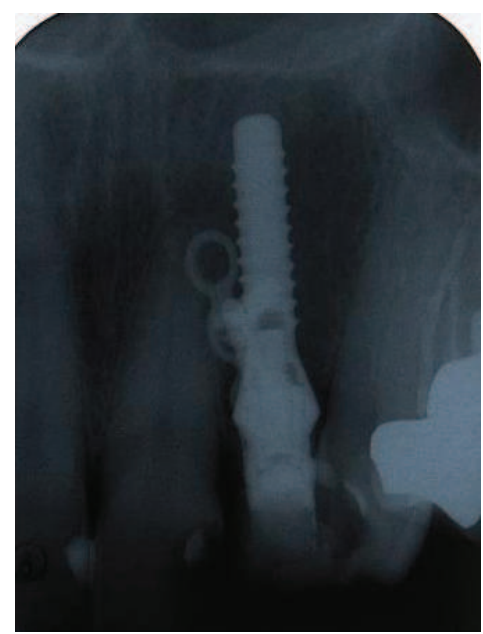

Abbildung 3.27: Postoperatives Röntgenbild

In der Abbildung 3.28 ist ein Einzelzahnfilm der Patientin 5 Jahre nach der Implantation zu sehen. Im Zuge der Nachuntersuchung ergab die Messung der Implantatstabilität mit dem Periotestgerät 0. Der horizontale Knochenabbau betrug mesial 1,3 und distal $0,9 \mathrm{~mm}$.

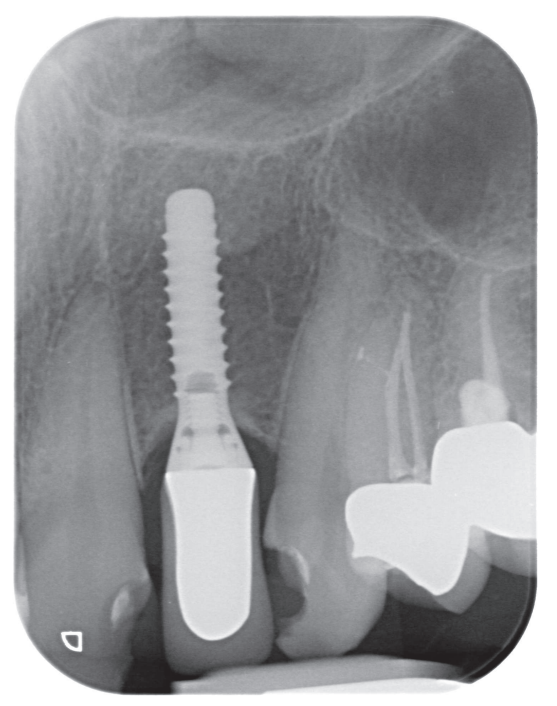

Abbildung 3.28: Röntgenbild 5 Jahre nach Implantation 
In den Abbildungen 3.29 und 3.30 ist die klinische Situation zum Zeitpunkt der Nachuntersuchung zu erkennen. Es sind keine klinischen Anzeichen einer Periimplantitis zu beobachten. Die Sondierungstiefen betrugen mesial und palatinal 2, vestibulär 1 und distal $3 \mathrm{~mm}$. Beim Sondieren trat keine Blutung auf. Der PPI betrug mesial und distal 3. Die Patientin benotete das Gesamtresultat ihrer Behandlung mit „sehr gut“.

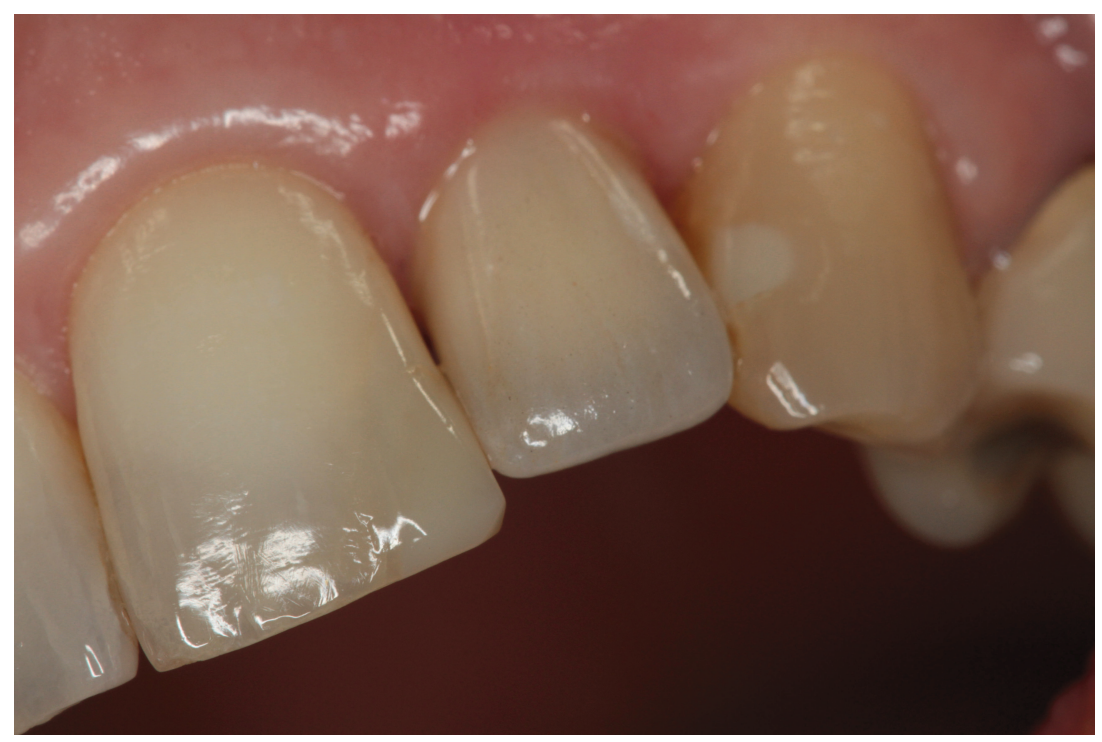

Abbildung 3.29: Ansicht von frontal 5 Jahre nach Implantation

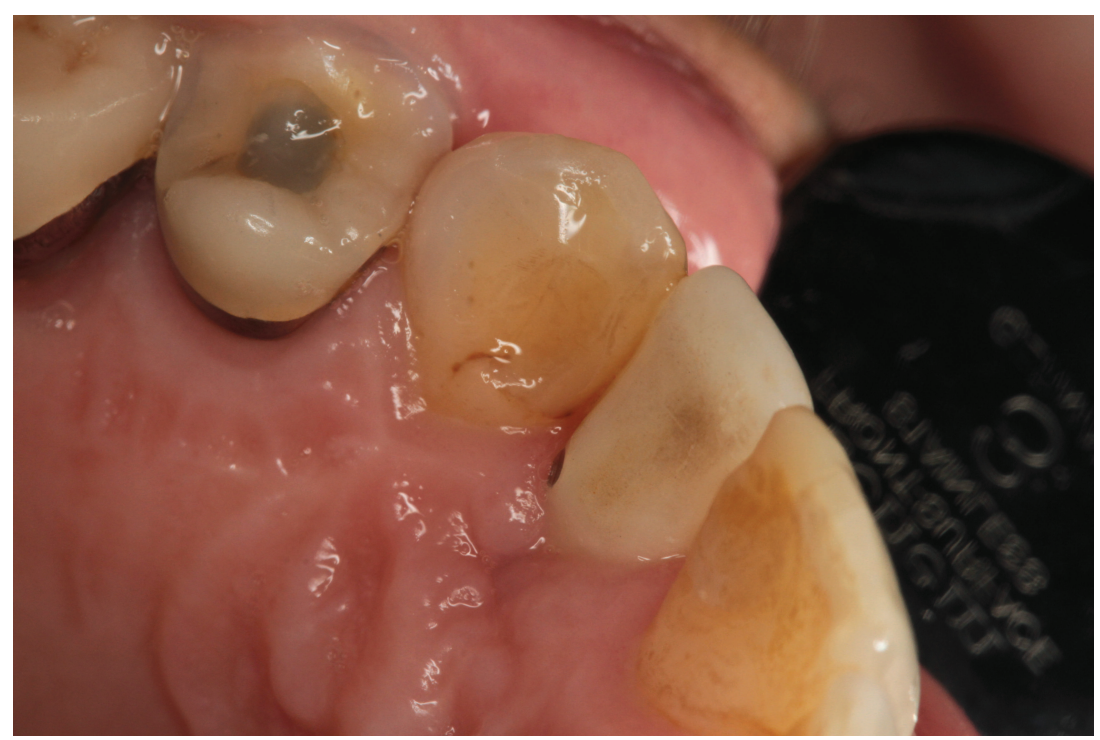

Abbildung 3.30: Ansicht von oral 5 Jahre nach Implantation

Bei der 40- jährigen zweiten Patientin wurde im April 2010 in einer Operation der nicht erhaltungswürdige Zahn 21 extrahiert und ein Implantat unverzüglich inseriert. Bei dem Implantat handelte es sich um ein Bone Level Implantat (Länge 12 mm, Durchmesser 4,1 mm) der Firma Straumann. Das Implantat wurde durch ein zusätzliches palatinales Satellitenimplantat stabilisiert, um eine sofortige Versorgung des Implantates zu 
ermöglichen (siehe Abb. 3.31). Es handelte sich hierbei um eine Osteosyntheseschraube der Länge $7 \mathrm{~mm}$. Das Satellitenimplantat war über eine 3-Loch-Osteosyntheseplatte mit dem Implantatabutment verbunden. Im Anschluss an die Implantation wurde das Implantat mit einer provisorischen Krone versorgt. Nach 6 Monaten und einer erfolgreichen Osseointegration des Zentralimplantates wurden die Satellitenimplantate wieder entfernt.

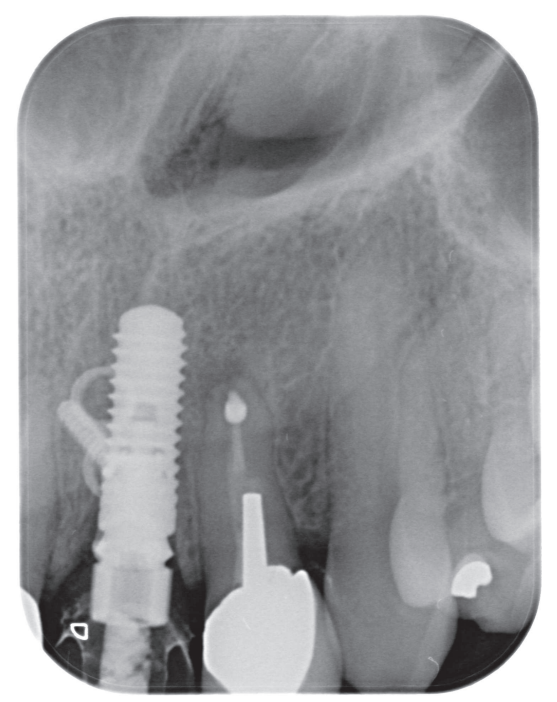

Abbildung 3.31: Postoperatives Röntgenbild

In der Abbildung 3.32 ist ein Einzelzahnfilm der Patientin 1 Jahr nach der Implantation zu sehen. Im Zuge der Nachuntersuchung ergab die Messung der Implantatstabilität mit dem Periotestgerät -1. Der horizontale Knochenabbau betrug mesial 0 und distal 0,3 mm. Bei der Nachuntersuchung wurde die Patientin auf die behandlungsbedürftige insuffiziente Wurzelkanalbehandlung mit apikalem Herd am Nachbarzahn hingewiesen.

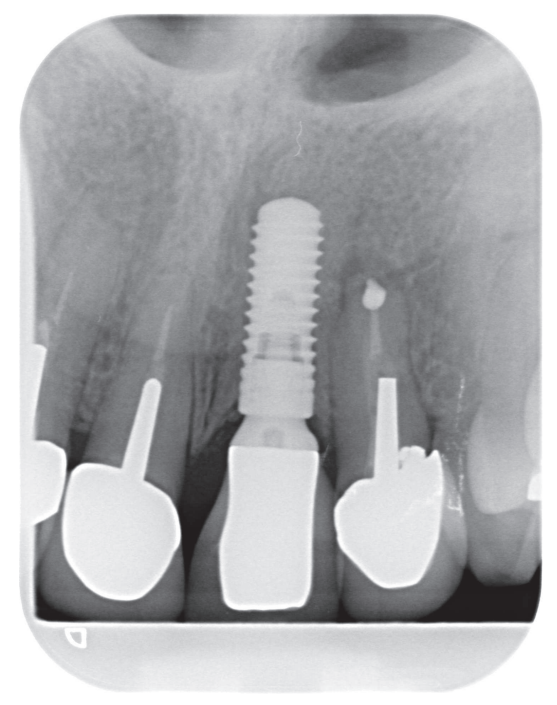

Abbildung 3.32: Röntgenbild 1 Jahr nach Implantation 
Die Abbildungen 3.33 und 3.34 zeigen die klinische Situation der Patientin zum Zeitpunkt der Nachuntersuchung. Keine klinischen Zeichen weisen auf eine Periimplantitis hin. Die Sondierungstiefen am Implantat betrugen mesial, vestibulär und palatinal 1 mm und distal $2 \mathrm{~mm}$. Beim Sondieren trat keine Blutung auf. Der PPI betrug mesial und distal 2. Die Patientin bewertete das Gesamtergebnis ihrer Behandlung als „gut“.

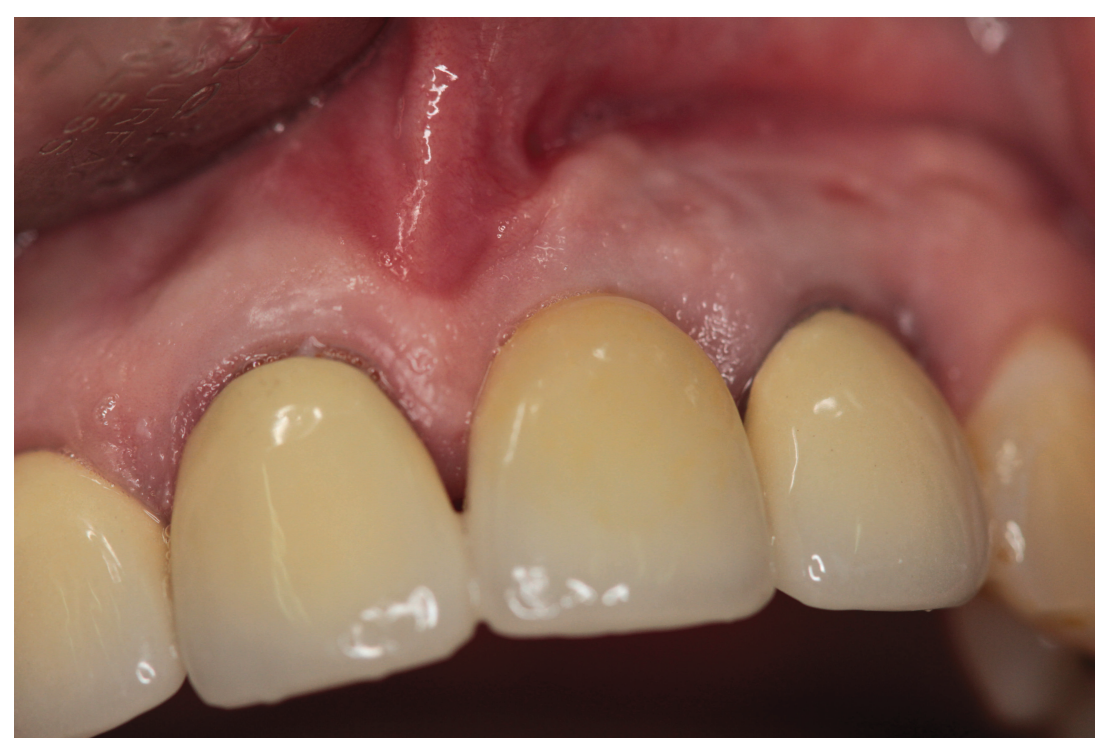

Abbildung 3.33: Ansicht von frontal 1 Jahr nach Implantation

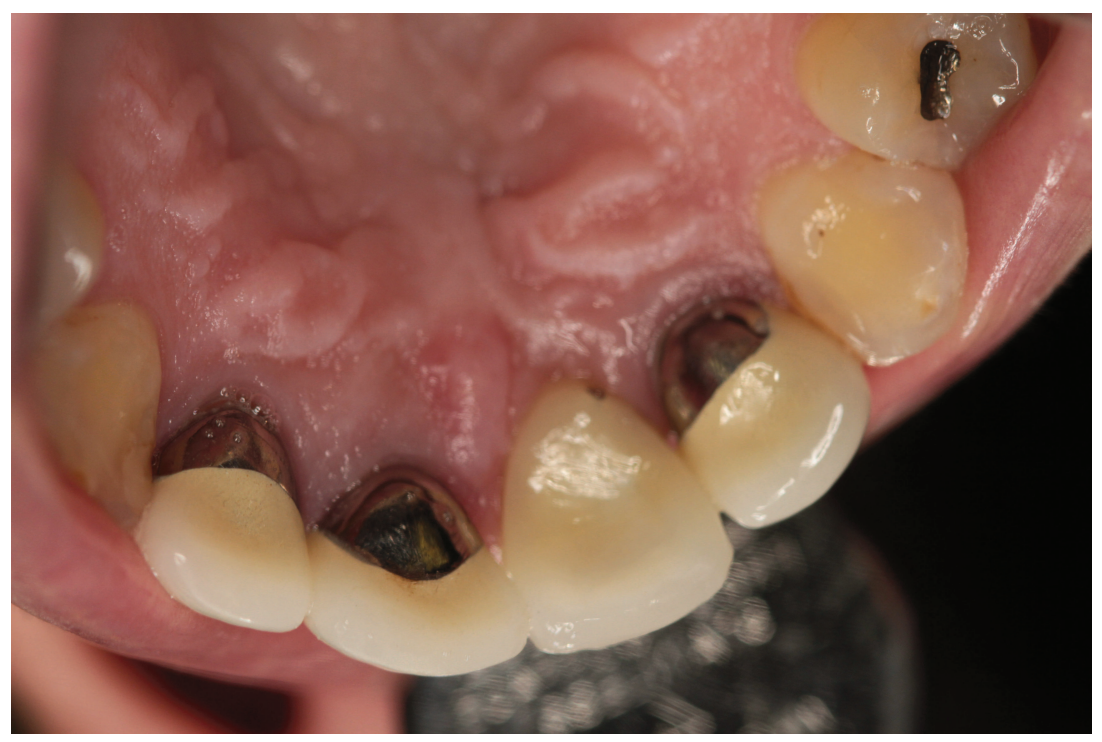

Abbildung 3.34: Ansicht von oral 1 Jahr nach Implantation 


\section{Diskussion}

\subsection{Beurteilung der Überlebensrate}

Im Rahmen dieser Studie konnte eine Gesamtüberlebensrate der Implantate von 98 \% ermittelt werden. Nach einem mittleren Beobachtungszeitraum von 46 Monaten trat 1 Implantatverlust auf. Die getrennte Betrachtung der Überlebensstatistiken für die beiden Implantationszeitpunkte ergab für die Sofortimplantate 96,97 \% und für die Spätimplantate $100 \%$. Dabei muss man beachten, dass das Patientenkollektiv der Gruppe der Sofortimplantate erheblich größer war (31 Patienten), als das der Spätimplantate (13 Patienten). Da nur ein Implantatverlust aufgetreten ist, konnte kein signifikanter Unterschied in den Überlebensraten der beiden Gruppen festgestellt werden.

Vergleicht man die in dieser Arbeit ausgewiesene Überlebensrate mit den Raten von entsprechenden Studien in der aktuellen Literatur, so findet man auch ähnliche Ergebnisse. Die Implantatüberlebensraten betrugen bei Cosyn et al. (2011) $96 \%$, bei Valentini et al. (2010) 95,3 \%, bei Laviv et al. (2010) 96,5 \%, bei Rouck et al. (2008) $97 \%$ und bei Palattella et al. (2008) $100 \%$.

Die Ergebnisse einiger Studien lassen vermuten, dass die Verlustrate bei sofort versorgten Sofortimplantaten womöglich höher ist als bei Spätimplantaten. Ribeiro et al. (2008) präsentierten Ergebnisse einer klinischen Studie, in der die Überlebensstatistiken von sofort versorgten Sofort- und Spätimplantaten verglichen wurden. Während in der Gruppe der Spätimplantate nach einem mittleren Beobachtungszeitraum von 39 Monaten kein Implantat verloren ging, traten in der Gruppe der Sofortimplantate 3 Implantatverluste auf. Ähnliches ergaben auch die Studien von Block et al. (2009), Laviv et al. (2010) und Raes et al. (2011), deren Ergebnisse in der Literaturübersicht (Tab. 1.3) präsentiert werden. In einer Metaanalyse von Grüttler und Belser (2009) wurde der klinische Erfolg von Implantaten in der Oberkieferfrontzahnregion unter Berücksichtigung des Belastungsprotokolls überprüft. In der Literaturanalyse stellten sie fest, dass die Überlebensraten für sofort versorgte und konventionell belastete Implantate nach einem Jahr 97,3 \% und nach 10 Jahren 96,7 \% betrugen. Betrachtete man jedoch die Überlebensrate für sofort implantierte und sofort belastete Implantate, fiel diese um $10 \%$ geringer aus. 
Zusammenfassend konnte man in dieser Arbeit für die sofort versorgten Implantate hohe Überlebensraten von über $95 \%$ ermitteln.

\subsection{Beurteilung der Patientenzufriedenheit}

Die Patientenzufriedenheit kann als einer der wichtigsten Faktoren für die Qualitätsicherung und den Behandlungserfolg einer Implantation betrachtet werden. Meyle und Mühlbrandt (1986) stellten fest, dass neben den objektiven klinisch messbaren Parametern die subjektive Akzeptanz eines der entscheidenden Kriterien für den Langzeiterfolg einer implantologischen Versorgung darstellt. Ein erfolgreich osseointegriertes Implantat garantiert nicht unbedingt eine hohe Zufriedenheit der Patienten. Daher ist das Erkennen und Erfassen der Patientenzufriedenheit essenziell für die abschließende Bewertung eines implantologischen Behandlungskonzeptes (Buch et al. 2002).

Zur Erfassung der Patientenzufriedenheit wurde im Rahmen dieser Studie ein Fragebogen verwendet. Der Fragebogen wurde von insgesamt 44 Patienten beantwortet. Dabei wurden 35 Befragungen im Zuge der klinischen Nachuntersuchung durchgeführt und 9 Befragungen telefonisch. Der Fragebogen bestand aus neun Fragen, von denen 5 durch eine Benotung nach dem deutschen Schulnotensystem und 3 durch Ja/Nein beantwortet werden sollten. Eine Frage wurde offen gestellt. Darüber hinaus sollten einige derselben Fragen auch anhand visueller Analogskalen beantwortet werden, um einen besseren Vergleich mit anderen internationalen Studien zu ermöglichen.

$98 \%$ der befragten Patienten benoteten das Gesamtresultat der Behandlung mit „sehr gut“ bis „gut". Nur $2 \%$ der Patienten vergaben die Schulnote „befriedigend“. Das Ergebnis der Befragung zeigt eine hohe Zufriedenheit der Patienten mit dem Behandlungskonzept der Sofortversorgung. Dieses Ergebnis wird auch durch die Fragen nach der Behandlungsempfehlung und der erneuten Behandlungswilligkeit bestätigt. So würden $95 \%$ der Befragten die Behandlung einem guten Bekannten oder Verwandten weiterempfehlen und sogar 98 \% sich bei Bedarf erneut behandeln lassen.

Die Ästhetik der implantologischen Versorgung wurde von $100 \%$ der Patienten mit „sehr gut" bis „gut" benotet. Im anbetracht der Tatsache, dass es sich um Implantate in der oberen Frontzahnregion handelt, kann das Ergebnis der Befragung als sehr erfreulich betrachtet werden.

Die funktionellen Aspekte der Implantate wurden zum Großteil auch sehr positiv bewertet. Die Abbeißfunktion und Hygienefähigkeit wurden von $93 \%$ der Patienten mit „sehr gut" bis ,gut" benotet. $91 \%$ der Befragten vergaben der Sprachfunktion die Noten „sehr gut" bis ,gut". 
Die Patienten gaben an, im Mittel nur einmal (MW 0,76) nach einem mittleren Beobachtungszeitraum von 46 Monaten aufgrund von Komplikationen einen Zahnarzt aufgesucht haben zu müssen. Die Betrachtung des Medianwertes (Median 0) zeigt, dass bei den meisten Patienten keinerlei Zahnarztbesuche aufgrund von Beschwerden nötig waren.

Im Mann-Whitney-U-Test konnten keine signifikanten Unterschiede in den Antworten der Patienten in Bezug auf den Implantationszeitpunkt (Sofort oder Spät) festgestellt werden.

Die Ergebnisse der Patientenbefragung in dieser Studie sind mit denen anderer klinischer Studien vergleichbar. In einer retrospektiven Studie von Hartmann und Steup (2005) wurden 17 Patienten nach Sofortimplantation und -versorgung in der Oberkieferfront gebeten, die Ästhetik und Funktion ihrer Versorgung mit einer deutschen Schulnote zu bewerten. Es wurde elfmal die Schulnote „sehr gut“ und sechsmal die Schulnote „gut“ gewählt. Den Hartog et al. (2011) benutzten eine visuelle Analogskala zur Quantifizierung der Patientenzufriedenheit mit dem Behandlungsergebnis von sofort versorgten Implantaten in der oberen Frontzahnregion. Das Gesamtresultat der Behandlung wurde von Patienten mit Sofortimplantaten durchschnittlich mit ca. 92 mm und von Patienten mit Spätimplantaten mit ca. $90 \mathrm{~mm}$ bewertet. Die präsentierten Ergebnisse von den Hartog et al. (2011) korrelieren mit denen in dieser Arbeit. Die Patienten markierten in dieser Studie durchschnittlich ca. $91 \mathrm{~mm}$ auf der visuellen Analogskala. Es konnten auch keine signifikanten Unterschiede zwischen der Zufriedenheit der Patienten mit Sofort- und Spätimplantaten beobachtet werden.

In der wissenschaftlichen Literatur sind viele Studien zu finden, die hohe Patientenzufriedenheiten mit den Resultaten von Frontzahnimplantationen dokumentieren. So werden meist Werte von über $88 \mathrm{~mm}$ auf visuellen Analogskalen angegeben, wenn 100 mm die höchst mögliche Zufriedenheit darstellen (Levi et al. (2003)).

In Bezug auf die Erfolgskriterien dentaler Implantate nach Jahn und d'Hoedt (1992) konnte diese Arbeit zeigen, dass die geforderte Patientenzufriedenheit erreicht wurde. Kein Patient vergab eine schlechtere Note als „befriedigend“. Zusammenfassend konnte man im Rahmen dieser Studie eine hohe Patientenakzeptanz des Konzeptes der sofort versorgten Implantate im Frontzahngebiet unter Anwendung kortikaler Mikrofixation feststellen. 


\subsection{Beurteilung der Sondierungstiefen und periimplantäten Gingiva}

Bei 40 Implantaten wurden im Rahmen der Nachuntersuchung zur Beurteilung der periimplantären Weichgewebsverhältnisse die Sondierungstiefen an vier verschiedenen Messpunkten bestimmt (mesial, distal, vestibulär und palatinal).

Die Sondierung der periimplantären Tasche wird in der Zahnmedizin als essentielles diagnostisches Mittel zum erkennen und erfassen entzündlicher Veränderungen am periimplantären Weich- und Hartgewebe angesehen. Mit dem Grad der periimplantären Entzündung nimmt auch die Eindringtiefe der Sonde und somit die ermittelte Taschentiefe zu (Lang et al. 1994). Folglich wird im Sinne einer Periimplantitisprophylaxe das vorsichtige Sondieren der periimplantären Tasche an 4 Seiten des Implantates empfohlen (Berger 2008). Dabei werden Sondierungstiefen bis 3,5 mm (Christensen et al. 1997) und das Fehlen einer Blutung nach Sondierung als „gesund“ betrachtet (Lang et al. 1990).

In dieser Studie wurden mittlere Sondierungstiefen von 2,25 $\mathrm{mm}$ mesial, 1,8 $\mathrm{mm}$ vestibulär, 1,72 $\mathrm{mm}$ palatinal und 2,3 $\mathrm{mm}$ distal gemessen. Es konnten keine aussagekräftigen Unterschiede bei den Sondierungstiefen der Sofort- und der Spätimplantate festgestellt werden. So waren die mittleren Sondierungstiefen bei der mesialen und distalen Messung bei den Sofortimplantaten etwas geringer als bei den Spätimplantaten. Bei der vestibulären und palatinalen Messung hingegen waren die Werte der Spätimplantate etwas geringer. In Bezug auf die zuvor genannten Erfolgskriterien nach Jahn und d'Hoedt (1992) (siehe Tabelle 2.4) kann man die mittleren Sondierungstiefen in dieser Studie als Erfolg betrachten.

Bei der Kovarianzanalyse dieser Werte konnte kein signifikanter Zusammenhang zwischen den Implantationszeitpunkt und den Sondierungstiefen festgestellt werden. Als Einflussfaktor konnte bei der Kovarianzanalyse das Vorhandensein eines erweiterten Parodontalspaltes am Nachbarzahn identifiziert werden ( $p$ von 0,0135). Auch die der Position der Sondierung hatte auf die Sondierungstiefe einen signifikanten Einfluss (p von 0,0001). So wurden mesial und distal der Implantate die höchsten Sondierungstiefen ermittelt. Die palatinale kortikale Mikrofixation hatte keinen negativen Einfluss auf die palatinalen Sondierungstiefen.

Der kritische Sondierungswert von $4 \mathrm{~mm}$ wurde bei einer Patientin überschritten. In diesem Fall betrug die Sondierungstiefe distal $5 \mathrm{~mm}$ und palatinal $6 \mathrm{~mm}$. Es lagen periimplantäre Entzündungszeichen wie eine stärkere Blutung auf Sondierung sowie radiologische Befunde einer Periimplantitis vor. Ein apikaler Herd war am benachbarten Zahn röntgenologisch nachweisbar. 
An 10 Implantaten traten bei der Sondierung Blutungen auf. Mit einer Ausnahme, handelte es sich um kleinere Blutungen. Dabei konnte kein signifikanter Zusammenhang zwischen den Blutungen und dem Implantationszeitpunkt nachgewiesen werden ( $p$ von 1). Die kleineren Blutungen können als Reizung der periimplantären Gingiva gewertet werden.

Vergleicht man die ermittelten periimplantären Sondierungstiefen mit denen in anderen Studien, findet man vergleichbare Ergebnisse. In einer prospektiven klinischen Studie von Cosyn et al. (2011) wurde nach einem mittleren Beobachtungszeitraum von 3 Jahren eine mittlere Sondierungstiefe von 3,17 mm für die sofort versorgten Sofortimplantate ermittelt. Rouck et al. (2008) ermittelten eine mittlere Sondierungstiefe von $3,46+/-0,69 \mathrm{~mm}$.

Zusammenfassend zeigen die Ergebnisse dieser Studie, dass die sofortige Versorgung eines Implantats sowie die Anwendung der kortikalen Mikrofixation die Sondierungstiefen nicht negativ beeinflussen.

\subsection{Beurteilung der Papillen}

Insbesondere bei implantologischen Restaurationen in der oberen Front ist ein ästhetisches Behandlungsergebnis für die Patienten von großer Bedeutung. Die Ästhetik von Frontzahnimplantaten wird dabei nicht nur durch das Erscheinungsbild der Zahnkronen selbst, sondern auch maßgeblich durch das des umgebenden Weichgewebes beeinflusst (Belser et al. (1998)). Vor allem der Präsenz einer Papille, die das interdentale Dreieck vollständig ausfüllt, wird in der Literatur eine entscheidende Rolle bei der Ästhetik zugeschrieben.

Um den Verlauf des periimplantären Weichgewebes zu beurteilen wurde im Rahmen dieser Studie der von Jemt (1997) entwickelte Papilla Presence Index angewendet. Der Medianwert der erhobenen PPI Werte betrug nach einem mittleren Beobachtungszeitraum von 46 Monaten insgesamt 2,00. Bei den Sofortimplantaten betrug der Medianwert 2,00, während der Medianwert der Spätimplantate 1,50 betrug. Trotz der besseren PPI Werte bei den Sofortimplantaten konnte in einer Kovarianzanalyse kein signifikanter Unterschied zwischen den PPI Werten der Sofort- und Spätimplantate festgestellt werden. Es konnte jedoch ein signifikanter Einfluss des Abstandes der Schmelzzementgrenze vom Limbus Alveolaris an den Nachbarzähnen auf den PPI Wert beobachtet werden $(\mathrm{p}<0,05)$, d.h. dass der PPI Wert durch den Zustand des benachbarten Hartgewebes beeinflusst wird.

In Bezug auf den PPI werden in der Literatur vergleichbare Resultate präsentiert. Somanathan et al. (2007) konnten ebenfalls bessere PPI Werte nach Sofortimplantationen 
als nach Spätimplantationen feststellen. 3 Monate nach definitiver prothetischer Versorgung wurde für die Sofortimplantate ein mittlerer PPI von 2,6 (mesial) bzw. 2,7 (distal) ermittelt und für die Spätimplantate ein mittlerer PPI von 1,5 (mesial) bzw. 1,7 (distal). Valentini et al. (2010) publizierten im Zuge einer Nachuntersuchung sofort versorgter Sofortimplantate auch sehr positive PPI Werte. Nach einem Jahr betrug der mittlere PPI 2,81. In einer prospektiven Studie von Joudzbalys und Wang (2007) betrug der PPI ein Jahr nach Sofortimplantation bei $64 \%$ der Papillen 2. Die restlichen Papillen wiesen einen optimalen PPI von 3 auf. Andere Studien konnten im Zuge mehrerer Nachuntersuchungen ein Wachstum der Papillen beobachten. Jemt und Lekholm (2003) ermittelten in einer klinischen Studie zum Zeitpunkt der prothetischen Implantatversorgung einen mittleren PPI von 1. Nach 2 Jahren wurden die periimplantären Papillen erneut beurteilt und es konnte ein mittlerer PPI von 2,4 ermittelt werden. Vor diesem Hintergrund wäre es interessant gewesen, PPI Werte zu haben, die direkt nach der Satellitenimplantation und Sofortversorgung erhoben wurden, um sie mit den erhobenen Werten nach einem Beobachtungszeitraum von 46 Monaten zu vergleichen.

In der aktuellen Literatur sind viele verschiedene Methoden zur Bewertung der periimplantären Weichgewebsverhältnisse zu finden. Einige prospektive klinische Studien dokumentierten die Kronenlänge der prothetischen Restauration zum Zeitpunkt der Versorgung und verglichen diese mit der Kronenlänge in späteren Recalluntersuchungen. So konnte man einen möglichen Verlust oder Gewinn an fazialer Gingivahöhe feststellen. Nach einem Beobachtungszeitraum von 2 Jahren stellten Block et al. (2009) fest, dass die Spätimplantate im Durchschnitt $1 \mathrm{~mm}$ mehr Verlust an fazialer Gingivahöhe aufwiesen als die Sofortimplantate.

Die These, dass durch eine sofortige Implantation und prothetische Versorgung nach Sofortimplantation ein besseres ästhetisches Ergebnis erzielt werden kann (Rouck et al. (2009)), scheint durch viele Studien unterstützt zu werden. Eine Tendenz zum besseren Papillenerhalt nach Sofortimplantation kann auch in dieser Studie beobachtet werden. Durch weiterführende Studien müssen letztendlich die Ergebnisse dieser Arbeit überprüft werden.

\subsection{Beurteilung der Implantatstabilität}

Der Erfolg einer Implantatbehandlung hängt im Wesentlichen von einer stabilen Verankerung des Implantates im Knochen ab. Daher stellt die Implantatstabilität einen der wichtigsten Parameter für die Implantatprognose dar (Brånemark (1983), Meredith (1998)). Die nicht invasive Beurteilung der Stabilität des Implantates ist daher ein essenzieller Bestandteil von Recalluntersuchung nach Implantationen. 
Die am häufigsten angewendeten Methoden zur Bestimmung der Implantatstabilität sind das Periotestverfahren (Medizintechnik Gulden, Bensheim, Deutschland) und die Resonanz-Frequenz-Analyse (Osstell Integration Diagnostics AB, Sävedalen, Schweden). In dieser Studie wurde das Periotestverfahren angewendet.

Nach Olive und Aparicio (1990) ist das Periotestverfahren eine einfach zu handhabende und objektive Messmethode. Laut Herstellerangaben dient das Verfahren der Beurteilung der Stabilität dentaler Implantate direkt nach der Implantation, nach erfolgter Implantateinheilung sowie nach koronaler prothetischer Versorgung, um etwaige negative Entwicklungen frühzeitig zu erkennen. Gemäß den Herstellerangaben und den Erfolgskriterien nach Jahn d'Hoedt (1992) soll ein erfolgreich osseointegriertes Implantat einen Periotestwert zwischen -8 und +8 haben.

Obwohl das Periotestverfahren in der Zahnmedizin als zuverlässiges Instrument zur Ermittlung der Implantatstabilität betrachtet wird, können einige Faktoren die Messwerte des Periotestgerätes beeinflussen. So zeigten Studien die Abhängigkeit der Periotestwerte von der vertikalen und horizontalen Lage des Messpunktes am Abutment und der Angulation des Handstücks bei der Messung (Olive und Aparicio (1990), Derhami et al. (1995), Meredith (1998)). Nur durch wiederholte Messungen während einer Untersuchung und eine ergänzende röntgenologische Auswertung der periimplantären Knochensituation können also zuverlässige und reproduzierbare Aussagen über die Implantatstabilität und Osseointegration gemacht werden (Cranin et al. 1998).

Im Zuge der Nachuntersuchung dieser Studie wurden bei 40 Implantaten nach einem mittleren Beobachtungszeitraum von 46 Monaten die Periotestwerte bestimmt. Dabei wurde die Messung aufgrund der festsitzenden koronalen Versorgung an der prothetischen Implantatkrone und nicht direkt am Implantatabutment durchgeführt. Insgesamt betrug der Mittelwert -0,02. Der Mittelwert für die Patienten mit Sofortimplantaten betrug 0,00 und für die mit Spätimplantaten -0,05. Es konnte kein signifikanter Unterschied zwischen den Werten bei den Sofort- und Spätimplantaten festgestellt werden (p von 0,947). Mit einer Ausnahme wurde bei keinem Implantat ein Wert größer als +5 gemessen.

Vergleicht man die Periotestwerte in dieser Studie mit denen in anderen Arbeiten, so gibt es Differenzen. Zum einen wurden in anderen Studien der ISQ aus der RFAMessung ermittelt, zum anderen wurden die Periotestwert-Messungen direkt am Implantatabutment und nicht an der prothetischen Versorgung durchgeführt. So veröffentlichten Lorenzoni et al. (2003) einen mittleren Periotestwert von -2 für sofort versorgte Implantate in der anterioren Maxilla. Dabei wurde die Messung 6 Monate nach Implantation am Implantatabutment durchgeführt. Zu diesem Zeitpunkt wurden die provisorischen Kronen durch die definitiven ersetzt.

Trotz der möglichen negativen Beeinflussung der Periotestwerte durch den vergrößerten 
Abstand des Periotesthandstücks zum Implantatabutment konnte in dieser Studie nur ein Implantat nicht die von Jahn und d'Hoedt (1992) geforderte Implantatstabilität von kleiner als +8 erfüllen. Mit der Ausnahme des einen Implantates konnte bei allen anderen Implantaten im Rahmen dieser Arbeit also eine befriedigende Implantatstabilität erreicht werden.

\subsection{Beurteilung des Knochenabbaus / -gewinns}

Die Ermittlung des periimplantären Knochenabbaus / -gewinns stellt einen wichtigen klinischen Parameter bei der Beurteilung des Erfolges einer Implantation dar. Als Ursachen des periimplantären Knochenabbaus werden in der Literatur verschiedene Faktoren angegeben. So sollen der Mikrospalt zwischen Abutment und Implantat (Hermann et al. (1997)), die mechanische (Über-) Belastung des Implantates (Misch (1995)), die bakterielle Besiedelung (Steflik et al. (1982)) und auch die Kompetenz und Erfahrung des Behandlers einen Einfluss auf das Ausmaß des Knochenabbaus haben (Albrektsson (2001)).

Zahlreiche Wissenschaftler haben Richtwerte zur Einschätzung des Knochenverlusts nach Implantationen veröffentlicht. In der internationalen Literatur wird der von Albrektsson et al. (1986) postulierte Wert von 0,2 mm Knochenverlust pro Jahr allgemein anerkannt. Die DGZMK betrachtet einen Knochenabbau von 0,5 $\mathrm{mm}$ in dem ersten Jahr nach Implantation sowie einen weiteren Knochenabbau von $0,2 \mathrm{~mm}$ in den folgenden Jahren als vertretbar (Neukam (2000)).

Die Bestimmung des horizontalen Knochenabbaus erfolgte in dieser Studie digital mit Hilfe einer speziellen Computersoftware. Für jede einzelne Röntgenaufnahme wurde dabei ein individueller Vergrößerungsfaktor berechnet. Als Referenz diente dabei die bekannte Implantatlänge.

Die Genauigkeit und Reproduzierbarkeit der röntgenologischen Bestimmung der periimplantären Knochenhöhe wird in der Literatur als relativ präzise bewertet. Smet et al. (2006) geben unabhängig von der Art der Röntgenaufnahme einen Messgenauigkeitsbereich von $0,5 \mathrm{~mm}$ an. Die digitale Vermessung wird meist der manuellen Vermessung bevorzugt (Borg et al. 2002). Sie wird nicht nur als untersucherfreundlicher sondern auch als untersucherunabhängiger betrachtet. Dabei zeigen Studien, dass die Messergebnisse der digitalen und manuellen Vermessung vergleichbar sind (Meijer et al. 1993, Borg et al. 2002, Henriksson et al. 2008).

Die Auswertung der radiologischen Parameter in dieser Studie ergab nach einem mittleren Beobachtungszeitraum von 46 Monaten einen mittleren Knochenverlust von 0,87 mm. Für die Sofortimplantate betrug der mittlere Knochenverlust 0,98 mm und für 
die Spätimplantate 0,71 mm. Bei 2 Implantaten aus der Gruppe der Sofortimplantationen konnte ein mittlerer Knochengewinn von 0,4 bis 0,9 $\mathrm{mm}$ beobachtet werden. Der Umfang dieser von parodontalen oder periostalen Strukturen ausgehende Hartgewebsneubildung liegt allerdings im Bereich der Messgenauigkeit der röntgenologischen Auswertung. In Bezug auf die zuvor genannten Richtwerte der DGZMK und Albrektsson et al. (1986) kann man den mittleren Knochenverlust in dieser Studie als Erfolg betrachten.

Bei der Auswertung der Röntgenbilder konnte bei 2 Implantaten aus der Gruppe der Sofortimplantationen ein mittlerer Knochenverlust von über $2 \mathrm{~mm}$ festgestellt werden. Eine periimplantäre Entzündung, ausgehend von einem apikalen Herd am Nachbarzahn, führte bei einer Patientin zu einem erhöhten mittleren Knochenverlust von 5,4 mm. Bei dem anderen Sofortimplantat hatte der Patient aus persönlichen Gründen die Entfernung des Satellitenimplantates und die Eingliederung einer definitiven prothetischen koronalen Restauration versäumt. 4 Jahre nach der Implantation konnte bei diesem Patienten ein mittlerer Knochenverlust von 2,9 mm ermittelt werden.

Im Rahmen einer Kovarianzanalyse wurden Faktoren, die den Knochenabbau möglicherweise beeinflussen könnten, berücksichtigt. In diesem Zusammenhang konnte kein signifikanter Einfluss des Implantationszeitpunktes auf den Knochenabbau festgestellt werden ( $\mathrm{p}$ von 0,7018 ). Des Weiteren zeigte die Kovarianzanalyse auch keinen signifikanten Einfluss der geprüften lokalen Risikofaktoren der Nachbarzähne.

Im Vergleich mit anderen aktuellen Studien, die die klinischen Ergebnisse sofort versorgter Frontzahnimplantate betrachten, sind die gemessenen Werte annähernd gleich groß. Cosyn et al. (2011) veröffentlichten nach einem Beobachtungszeitraum von 3 Jahren einen mittleren marginalen Knochenabbau von mesial 1,13 mm und distal 0,86 mm. Nach einem Beobachtungsintervall von 18 Monaten konnten den Hartog et al. (2011) einen mittleren Knochenabbau von 0,91 mm feststellen. In einer Studie von Mijiritsky et al. (2009) wurde nach 6 Jahren ein mittlerer marginaler Knochenverlust von 0,9 mm gemessen.

Andere Studien richteten ihr Augenmerk auf den Vergleich des Knochenabbaus um sofort versorgte Sofort- und Spätimplantate. Tsirlis (2005) konnte nach 2 Jahren bei den Sofortimplantaten einen mittleren Knochenverlust von 0,75 $\mathrm{mm}$ und bei den Spätimplantaten einen etwas größeren mittleren Knochenverlust von $0,87 \mathrm{~mm}$ feststellen. Im Gegensatz dazu stellten Palattella et al. (2008) bei den Sofortimplantaten einen geringfügig größeren Knochenabbau fest, was auch den Ergebnissen in dieser Studie entspricht. Nichtdestotrotz muss man berücksichtigen, dass das Patientenkollektiv der Sofortimplantate in dieser Studie wesentlich größer war als das der Spätimplantate, sodass es keinen aussagekräftigen Unterschied in Bezug auf den Knochenabbau zwischen den beiden Gruppen gab. Darüberhinaus konnte im Zuge dieser Studie nur 
bei den Sofortimplantaten auch ein Knochengewinn beobachtet werden.

Die Ergebnisse dieser Studie zeigen, dass die Anwendung kortikaler Schrauben zur Fixierung eines Zentralimplantates keinen negativen Einfluss auf den periimplantären Knochenabbau hatte und mit den klinischen Ergebnissen anderer Studien korreliert. Dabei scheint die Voraussetzung für eine erfolgreiche Anwendung der kortikalen Mikrofixation die zeitgerechte Entfernung der Satellitenimplantate nach erfolgreicher Osseointegration der Zentralimplantate zu sein.

\subsection{Beurteilung der Erfolgsquote}

Obwohl diverse Autoren klinische Parameter vorgeschlagen haben um ein Implantationsergebnis zu bewerten, gibt es für die Definition eines Implantaterfolges bis heute noch keinen internationalen Konsens. Vor diesem Hintergrund ist ein zuverlässiger Vergleich von Studienergebnissen erschwert (Buch et al. 2003).

Bereits 1978 stellten Schnitman und Shulmann auf der National Institutes of Health Consensus Development Conference Erfolgskriterien vor (Schnitman und Shulmann 1979). Die Implantatmobilität solle weniger als $1 \mathrm{~mm}$ und der Knochenabbau weniger als ein Drittel der Implantatlänge betragen. Die Okklusion solle stabil sein und das Implantat solle keine Anzeichen einer therapieresistenten Infektion besitzen. Des Weiteren solle keine Verletzung von Nachbargeweben vorliegen.

In den folgenden Jahren wurden von weiteren Wissenschaftlern, wie Albrektsson et al. (1986), Buser et al. (1990), Jahn und d'Hoedt (1992), Naert et al. (1992), Roos et al. (1997), Snauwaert et al. (2000) und Misch et al. (2008) zahlreiche weitere Kriterien vorgeschlagen. Laut den Richtlinien der DGZMK soll ein erfolgreiches Implantat sich klinisch fest, entzündungsfrei, schmerzfrei und ohne periimplantäre Osteolyse in situ befinden (Neukam (2000)).

Im Rahmen der Arbeitsgruppe der Abteilung Mund-, Kiefer- und Gesichtschirurgie der Universitätsmedizin Göttingen entschied man sich in dieser Studie für die Anwendung der Erfolgskriterien nach Jahn und d'Hoedt (1992) und Naert et al. (1992) (siehe Tab. 2.4 ). Die Erfolgsquoten nach Jahn und d'Hoedt (1992) und nach Naert et al. (1992) betrugen jeweils 97,5 \%. Obwohl die Kriterien nach Jahn und d'Hoedt (1992), im Gegensatz zu denen nach Naert et al. (1992), die subjektive Benotung des Implantates durch die Patienten berücksichtigen, sind die Erfolgsquoten identisch. Kein Patient bewertete das Gesamtergebnis seiner Implantatbehandlung schlechter als „befriedigend“. 


\subsection{Schlussfolgerung}

Nach einem mittleren Beobachtungszeitraum von 46 Monaten liefert das Konzept der implantologischen Sofortversorgung mit palatinaler subgingivaler kortikaler Mikrofixation in der oberen Frontzahnregion positive Ergebnisse. Es zeigt sowohl für Sofort- als auch Spätimplantationen hohe Überlebensraten von über 95 \%. Ein negativer Einfluss auf die periimplantären Hart- und Weichgewebsverhältnisse konnte bei zeitgerechter Entfernung der Satellitenimplantate innerhalb dieses Beobachtungsintervalls nicht beobachtet werden. Das Behandlungskonzept und die Ästhetik der Versorgung wurden von der Mehrheit der Patienten als positiv und empfehlenswert empfunden. Durch die Behandlung wurde eine hohe Patientenzufriedenheit erreicht. Weiterführende Studien müssen diese Ergebnisse noch bestätigen. 


\section{Zusammenfassung}

Ziel dieser retrospektiven Studie war es, nach einem mittleren Beobachtungszeitraum von 46 Monaten den Erfolg des Behandlungskonzeptes der Sofortversorgung von oberen Frontzahnimplantaten unter Anwendung palatinaler kortikaler Satellitenimplantate zu überprüfen.

An dieser Studie nahmen 44 Patienten (29 Frauen und 15 Männer, Durchschnittsalter 58 Jahre) teil, die im Zeitraum von 2000 bis 2010 in der oberen Frontzahnregion mit Implantaten unter Anwendung der palatinalen kortikalen Mikrofixation versorgt wurden. Durch die Verwendung der palatinalen subgingivalen Satellitenimplantate wurde das eigentliche Zentralimplantat über einen Konnektor stabilisiert und eine sofortige koronale Versorgung des Zentralimplantates ermöglicht. Bei den Zentralimplantaten handelte es sich um Schraubenimplantate der Firma Straumann und der Firma Bego. Von den 52 inserierten Implantaten waren 33 Sofortimplantate und 19 Spätimplantate. Alle Studienteilnehmer nahmen an der Befragung teil und 35 Patienten an der klinischen Nachuntersuchung. Die Nachuntersuchung fand im Zeitraum von März bis Juli 2011 statt.

Nach einem mittleren Beobachtungszeitraun von 46 Monaten betrug die Gesamtüberlebensrate der Implantate 98 \%. Die Überlebensrate für die Sofortimplantate betrug 96,97\% und für die Spätimplantate $100 \%$.

Insgesamt konnte ein mittlerer Knochenverlust von 0,87 mm ermittelt werden. Für die Sofortimplantate betrug der mittlere Knochenverlust $0,98 \mathrm{~mm}$ und für die Spätimplantate $0,71 \mathrm{~mm}$.

Bei der Beurteilung der periimplantären Weichgewebsverhältnisse wurden mittlere Sondierungstiefen von 1,72 bis 2,3 mm ermittelt. Der ermittelte Medianwert des PPIIndices betrug insgesamt 2,0. Ein Medianwert von 2,0 konnte bei den Sofortimplantaten und einer von 1,5 bei den Spätimplantaten beobachtet werden.

Die Auswertung der Implantatstabilität ergab einen mittleren Periotestwert von -0,02. Der Mittelwert für die Patienten mit Sofortimplantaten betrug 0,00 und für die mit Spätimplantaten $-0,05$.

$98 \%$ der Patienten benoteten das Gesamtresultat der Behandlung mit ,sehr gut" bis „gut“. Die restlichen 2 \% vergaben die Note ,befriedigend“. Die Ästhetik der Behandlung 
wurde von $100 \%$ der Patienten mit ,sehr gut“ bis ,gut“ bewertet. $98 \%$ der Befragten würden sich bei Bedarf erneut behandeln lassen. Es konnten keine Unterschiede in der Zufriedenheit der Patienten mit Sofort- und Spätimplantaten festgestellt werden. 


\section{Anhang}

\subsection{Abkürzungsverzeichnis}

$\begin{array}{ll}\text { Abb. } & \text { Abbildung } \\ \text { Abs. } & \text { Abschnitt } \\ \text { ANOVA } & \text { Analysis of Variance } \\ \text { BDIZ } & \text { Bundesverband der implantologisch tätigen Zahnärzte } \\ \text { cm } & \text { Zentimeter } \\ \text { DGZMK } & \text { Deutsche Gesellschaft für Zahn-, Mund- und Kieferheilkunde } \\ \text { EuCC } & \text { Europäische Konsensuskonfernez Implantologie } \\ \text { ISQ } & \text { Implant Stability Quotient } \\ \text { ITI } & \text { International Team for Implantology } \\ \text { Konv.Bel. } & \text { Konventionelle Belastung } \\ \text { Max. } & \text { Maximum } \\ \text { Min. } & \text { Minimum } \\ \text { mm } & \text { Millimeter } \\ \text { MW } & \text { Mittelwert } \\ \text { N } & \text { Anzahl } \\ \text { NZ } & \text { Nachbarzahn } \\ \text { PA-Spalt } & \text { Parodontalspalt } \\ \text { PES } & \text { Pink Esthetic Score } \\ \text { RFA } & \text { Papilla Presence Index } \\ \text { Resonanz-Frequenz-Analyse } \\ \text { Seite }\end{array}$


sign. signifikant

Sofortbel. Sofortbelastung

Sofortimpl. Sofortimplantate

Sofortvers. Sofortversorgung

Spätimpl. Spätimplantate

Std.abw. Standardabweichung

SZG Schmelzzementgrenze

Tab. Tabelle

WES White Esthetic Score

\subsection{Tabellenverzeichnis}

1.1 Literaturübersicht der Sofortversorgung von 2005-2007 . . . . . . . . . . . . . . 8

1.2 Literaturübersicht der Sofortversorgung von 2008-2009 _ . . . . . . . . . . . . . 9

1.3 Literaturübersicht der Sofortversorgung von 2009-2011 . . . . . . . . . . . . . . . 10

1.4 Literaturübersicht der Sofortversorgung von 2011 . . . . . . . . . . . . . . . . . . 11

2.1 Veranschaulichung des Patientenkollektivs. . . . . . . . . . . . . . . . . . . 19

2.2. Charakteristika der verwendeten Implantate . . . . . . . . . . . . . . . . . . 21

2.4 International anerkannte Erfolgskriterien für enossale dentale Implantate . . . . . 29

3.1 Gesamtresultat der Behandlung (deutsche Schulnote) . . . . . . . . . . . . 32

3.2 Beurteilung des Gesamtresultates (visuelle Analogskala $(\mathrm{mm})$ ) . . . . . . . . . . 32

3.3 Bewertung der Ästhetik (deutsche Schulnote) . . . . . . . . . . . . . . . . .33

3.4 Bewertung der Ästhetik (visuelle Analogskala $(\mathrm{mm})$ ) . . . . . . . . . . . . . . 33

3.5 Bewertung Abbeißfunktion (deutsche Schulnote) . . . . . . . . . . . . 35

3.6 Bewertung der Abbeiffunktion (visuelle Analogskala $(\mathrm{mm})$ ) . . . . . . . . . . 35

3.7 Bewertung Sprachfunktion (deutsche Schulnote) . . . . . . . . . . . 36

3.8 Bewertung der Sprachfunktion (visuelle Analogskala $(\mathrm{mm})$ ) . . . . . . . . . . .36

3.9 Bewertung der Hygienefähigkeit (deutsche Schulnote) . . . . . . . . . . . . 38

3.10 Anzahl der Zahnarztbesuche aufgrund von Beschwerden . . . . . . . . . . .38

3.11 Behandlungsempfehlung $(\mathrm{Ja} / \mathrm{Nein}) \ldots \ldots \ldots \ldots \ldots \ldots$. . . . . . . . . . 40 
3.12 Behandlungsempfehlung (visuelle Analogskala $(\mathrm{mm})$ ) . . . . . . . . . . . . 40

3.13 Erneute Behandlungswilligkeit $(\mathrm{Ja} / \mathrm{Nein}) \ldots \ldots \ldots \ldots \ldots \ldots \ldots \ldots$

3.14 Erneute Behandlungwilligkeit (visuelle Analogskala $(\mathrm{mm})$ ) . . . . . . . . . . .42

3.15 Zufriedenheit mit Mundgesundheit $(\mathrm{Ja} /$ Nein $) \ldots \ldots \ldots \ldots \ldots \ldots$

3.16 Zufriedenheit mit Mundgesundheit (visuelle Analogskala $(\mathrm{mm})$ ) . . . . . . . . 43

3.17 Risikofaktoren . . . . . . . . . . . . . . . . . . . . . . . . . . . . . . . 44

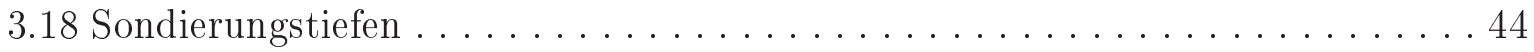

3.19 Ergebnis der Kovarianzanalyse der Sondierungstiefen . . . . . . . . . . . . 47

3.20 Ergebnis der Bonferroni-Adjustierung der Kovarianzanalyse . . . . . . . . . . . .47

3.21 Übersicht PPI-Werte . . . . . . . . . . . . . . . . . . . . . . . 48

3.22 Ergebnis der Kovarianzanalyse der PPI-Werte . . . . . . . . . . . . . .50

3.23 Übersicht der Periotestwerte . . . . . . . . . . . . . . . . . . . . . . . . . . 50

3.24 Messdaten des horizontalen Knochenabbaus/-gewinns . . . . . . . . . . . . . .51

3.25 Ergebnis der Kovarianzanalyse des Knochenabbaus/-gewinns . . . . . . . . . . .53

\subsection{Abbildungsverzeichnis}

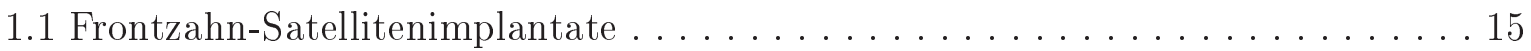

2.1 Semados Implantat (Malinski $(2011)$ ) . . . . . . . . . . . . . . . 20

2.2 Straumann Implantate (Bone Level, Standard, Standard Plus) . . . . . . . . . . . 21

2.3 Satellitenimplantatabutment und Konnektor (Dr. Möller) . . . . . . . . . . . .22

2.4 Provisorisches Abutment mit befestigten Konnektor (Dr. Möller) . . . . . . . . . 23

2.5 Zustand nach Entfernung eines Satellitenimplantates (Dr. Möller) . . . . . . . . 23

2.6 Berücksichtigung der individuellen Dimensionsänderung . . . . . . . . . . . .27

2.7 Bestimmung des mesialen und distalen Knochenabbaus . . . . . . . . . . . . . 27

3.1 Diagramm Beurteilung des Gesamtresultates der Behandlung . . . . . . . . . 31

3.2 Boxplot Gesamtresultat der Behandlung . . . . . . . . . . . . . . . . . . . . . 32

3.3 Diagramm Bewertung der Ästhetik . . . . . . . . . . . . . . . . . . 33

3.4 Boxplot Bewertung der Ästhetik . . . . . . . . . . . . . . . . . . . . 34

3.5 Diagramm Bewertung der Abbeißfunktion . . . . . . . . . . . . . . 34 


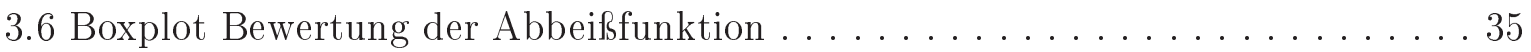

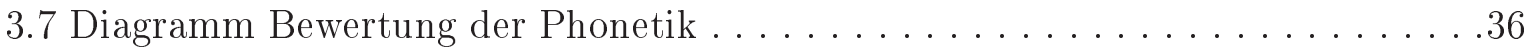

3.8 Boxplot Beurteilung der Sprachfunktion . . . . . . . . . . . . . . . . . . . 37

3.9 Diagramm Bewertung der Hygienefähigkeit . . . . . . . . . . . . . . . 37

3.10 Diagramm Anzahl der Zahnarztbesuche aufgrund von Beschwerden . . . . . . 38

3.11 Boxplot Anzahl der Zahnarztbesuche aufgrund von Beschwerden . . . . . . . . .39

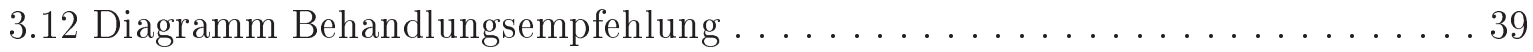

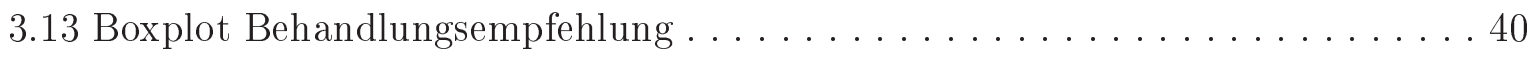

3.14 Diagramm erneute Behandlungswilligkeit . . . . . . . . . . . . . . . . 41

3.15 Boxplot Erneute Behandlungswilligkeit . . . . . . . . . . . . . . . . . 42

3.16 Diagramm Zufriedenheit mit Mundgesundheit . . . . . . . . . . . . . . . 42

3.17 Boxplot Zufriedenheit mit Mundgesundheit . . . . . . . . . . . . . . . . .43

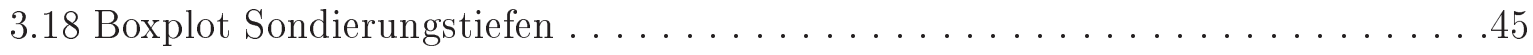

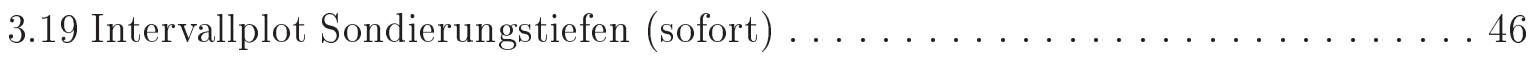

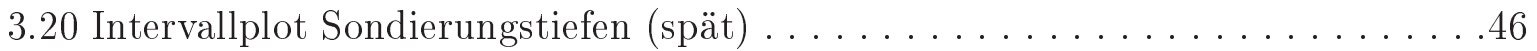

3.21 Säulendiagramm Blutung auf Sondierung $(\mathrm{Ja} / \mathrm{Nein}) \ldots \ldots \ldots$. . . . . . . . 48

3.22 Boxplot PPI-Werte (gesamt) . . . . . . . . . . . . . . . . . . . 49

3.23 Boxplot PPI-Werte (sofort/spät) . . . . . . . . . . . . . . . . . . . . .49

3.24 Boxplot Knochenabbau/-gewinn (gesamt) . . . . . . . . . . . . . . . 51

3.25 Boxplot Knochenabbau/-gewinn (sofort/spät) . . . . . . . . . . . . . . 52

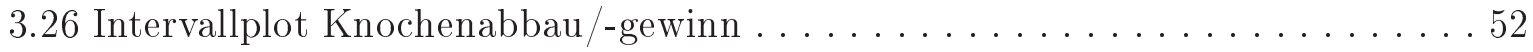

3.27 Postoperatives Römtgenbild . . . . . . . . . . . . . . . . . . 54

3.28 Röntgenbild 5 Jahre nach Implantation. . . . . . . . . . . . . . . . . 54

3.29 Ansicht von frontal 5 Jahre nach Implantation $\ldots \ldots \ldots \ldots \ldots \ldots \ldots \ldots$

3.30 Ansicht von oral 5 Jahre nach Implantation . . . . . . . . . . . . . . 55

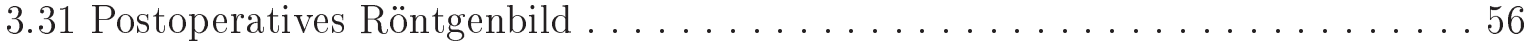

3.32 Röntgenbild 1 Jahre nach Implantation. . . . . . . . . . . . . . 56

3.33 Ansicht von frontal 1 Jahr nach Implantation . . . . . . . . . . . . . . 57

3.34 Ansicht von oral 1 Jahr nach Implantation . . . . . . . . . . . . . . . 57 


\section{Literaturverzeichnis}

Achilli A, Tura F, Euwe E (2007): Immediate/early function with tapered implants supporting maxillary and mandibular posterior fixed partial dentures: Preliminary results of a prospective multicenter study. J Prosthet Dent 97, 52-58

Albrektsson T (2001): Is surgical skill more important for clinical success than changes in implant hardware? Clin Implant Dent Relat Res $\underline{3}$, 174-175

Albrektsson T, Zarb G, Worthington P, Eriksson AR (1986): The long-term efficacy of currently used dental implants: a review and proposed criteria of success. Oral Maxillofac Implants $1,11-25$

Baumgarten H, Cocchetto R, Testori T, Meltzer A, Porter S (2005): A new implant design for crestal bone preservation: initial observations and case report. Pract Proced Aesthet Dent 17, 735-740

Becker CM, Wilson TG, Jensen OT (2011): Minimum criteria for immediate provisionalization of single-tooth dental implants inextraction sites: a 1-year retrospective study of 100 consecutive cases. J Oral Maxillofac Surg 69, 491-497

Bedard JF (2009): Enhanced cast-based guided dental implant placement for ultimate esthetics: concept and technical procedures. J Oral Maxillofac Surg 67, 108-114

Behr M, Leibrock A, Stich W, Rammelsberg P, Rosentritt M, Handel G (1998): Adhesivefixed partial dentures in anterior and posterior areas. Results of an ongoing prospective study begun in 1985. Clin Oral Investig 2, 31-35

Belser UC, Buser D, Hess D, Schmid B, Bernard JP, Lang NP (1998): Esthetic implant restorations in partially edentulous patients - a critical appraisel. Periodontology $\underline{17}$, $132-150$

Belser UC, Schmid B, Higginbottom F, Buser D (2004): Outcome analysis of implant restorations located in the anterior maxilla: a review of the recent literature. Int J Oral Maxillofac Implants $\underline{19}$, 30-42

Berger C: Konsenspapier BDIZ. Sofortversorgung und Sofortbelastung auf Implantaten. European Consensus Conference (EuCC), Köln 2006 
Berger C: Konsenspapier BDIZ. Periimplantitis: Prävention-Diagnostik-Therapie. 3. European Consensus Conference (EuCC), Köln 2008

Block MS, Mercante DE, Lirette D, Mohamed W, Ryser M, Cystellon P (2009): Prospective evaluation of immediate and delayed provisional single tooth restorations. J Oral Maxillofac Surg 67, 89-107

Borg E, Gröndahl K, Persson LG, Gröndahl HG (2002): Marginal bone level around implants assessed in digital and film radiographs: In vivo study in the dog. Dentomaxillofac Radiol 31, 176-181

Brånemark PI (1983): Osseointegration and its experimental background. J Prosthet Dent $\underline{50}, 399-410$

Brånemark PI, Svensson B, Steenberghe D (1995): Ten-year survival rates of fixed prostheses on four or six implants ad modum Brånemark in full edentulism. Clin Oral Implants Res $\underline{6}, 227-231$

Buch RSR, Weibrich G, Wegener J, Wagner W (2002): Patient satisfaction with dental implants. Mund Kiefer Gesichtschir $\underline{6}$, 433-436

Buch R, Weibrich G, Wagner G (2003): Erfolgskriterien in der Implantologie. Mund Kiefer Gesichtschir 푸 42-46

Buser D, von Arx T (2000): Surgical procedures in partially edentulous patients with ITI implants. Clin Oral Implants Res 11, 83-100

Buser D, Weber HP, Lang NP (1990): Tissue integration of non-submerged implants. 1-year results of a prospective study with 100 ITI hollow-cylinder and hollow-screw implants. Clin Oral Implants Res 1, 33-40

Buser D, Mericske-Stern R, Bernard JP, Behneke A, Behneke N, Hirt HP, Belser UC, Lang NP (1997): Long-term evaluation of non-submerged ITI implants. Part 1: 8-year life table analysis of a prospective multi-center study with 2359 implants. Clin Oral Implants Res $\underline{8}, 161-172$

Buser D, Martin W, Belser UC (2004): Optimizing esthetics for implant restorations in the anterior maxilla: anatomic and surgical considerations. Int J Oral Maxillofac Implants $\underline{19}, 43-61$

Buser D, Chen ST, Weber HP, Belser UC (2008): Early implant placement following single-tooth extraction in the esthetic zone: biologic rationale and surgical procedures. Int J Periodont Rest Dent 28, 441-451 
Canullo L, Rasperini G (2007): Preservation of peri-implant soft and hard tissues using platform switching of implants placed in immediate extraction sockets: a proof-ofconcept study with 12- to 36-month follow-up. Int J Oral Maxillofac Implants 22, 995-1000

Chaushu G, Chaushu S, Tzohar A, Dayan D (2001): Immediate loading of single-tooth implants: immediate versus non-immediate implantation. A clinical report. Int J Oral Maxillofac Implants 16, 267-272

Christensen MM, Joss A, Lang NP (1997): Reproducibility of automated periodontal probing around teeth and osseointegrated oral implants. Clin Oral Implants Res $\underline{8}$, $455-464$

Cochran DL, Schenk RK, Lussi A, Higginbottom FL, Buser D (1998): Bone response to unloaded and loaded titanium implants with a sandblasted and acid-etched surface: a histometric study in the canine mandible. J Biomed Mater Res $\underline{40,}$ 1-11

Cochran DL, Monton D, Weber HP (2004): Consensus statements and recommended clinical procedures regarding loading protocols for endosseous dental implants. Int J Oral Maxillofac Implants 19, 109-113

Cosyn J, Eghbali A, Bruyn HD, Collys K, Cleymaet R, Rouck TD (2011): Immediate single-tooth implants in the anterior maxilla: 3-year results of a case series on hard and soft tissue response and aesthetics. J Clin Periodontol 38, 746-753

Cranin AN, DeGrado J, Kaufman M, Baraoidan M, DiGrigorio R, Batgitis G, Lee ZB (1998): Evaluation of the Periotest as a diagnostic tool for dental implants. J Oral Implantol 24, 139-146

Crespi R, Cappare P, Gherlone E, Romanos GE (2008): Immediate versus delayed loading of dental implants placed in fresh extraction sockets in the maxillary esthetic zone: a clinical comparative study. Int J Oral Maxillofac Implants 23, 753-758

Danza M, Fanali S, Quaranta A, Vozza I (2010): The importance of immediate postextractive implants in esthetical rehabilitation: case series. Minerva Stomatol $\underline{59}, 215-$ 220

den Hartog L, Raghoebar GM, Stellingsma K, Vissink A, Meijer HJ (2011): Immediate non-occlusal loading of single implants in the aesthetic zone: a randomized clinical trail. J Clin Periodontol 38, 186-194

d'Hoedt B, Lukas D, Mühlbradt L, Scholz F, Schulte W, Quante F, Topkaya A (1985): [Periotest methods-development and clinical trial]. Dtsch Zahnarztl Z 40, 113-125 
Derhami K, Wolfaardt JF, Faulkner G, Grace M (1995): Assessment of the periotest device in baseline mobility measurements of craniofacial implants. Int J Oral Maxillofac Implants $\underline{10}, 221-229$

Elias AC, Sheiham A (1998): The relationship between satisfaction with mouth and number, position and condition of teeth: studies in Brazilian adults. J Oral Rehabil 26, $53-71$

Engelke W, Stahr S, Schwarzwäller W (2002): Erhöhung der Primärstabilität dentaler Implantate durch Satellitenimplantate. Eine in Vitro-Untersuchung. Oralchir J 2, 12-15

Engelke W, Decco OA, Cura AC, Ruscio ML (2011): Immediate loading of freestanding implants using cortical satellite implants: 3-year results of an ongoing prospective study. Implant Dent 20, 341-344

Esposito M, Grusovin MG, Martinis E, Coulthard P, Worthington HV (2007): Interventions for replacing missing teeth: 1- versus 2-stage implant placement. Cochrane Database Syst Rev 3, CD006698

Esposito M, Grusovin MG, Polyzos IP, Felice P, Worthington HV (2010): Timing of implant placement after tooth extraction: immediate, immediate-delayed or delayed implants? Cochrane Syst Rev. Eur J Oral Implantol 3, 189-205

Fürhauser R, Florescu D, Benesch T, Haas R, Mailath G, Watzek G (2005): Evaluation of soft tissue around single-tooth implant crowns: the pink esthetic score. Clin Oral Implants Res 16, 639-644

Gaggl A, Schultes G, Rainer H, Karcher H (2002): The transgingival approach for placement of distraction implants. J Oral Maxillofac Surg _60, 793-796

Gibbard LL, Zarb G (2002): A 5-year prospective study of implant- supported singletooth replacements. J Can Dent Assoc 68, 110-116

Gomez-Roman G (2001): Influence of flap design on peri-implant interproximal crestal bone loss around single-tooth implants. Int J Oral Maxillofac Implants 16, 61-67

Grüttler L, Belser UC (2009): Implant loading protocols for the partially edentulous esthetic zone. Int J Oral Maxillofac Implants 24, 169-179

Hartmann HJ, Steup A (2005): Ästhetische Langzeitergebnisse der Sofortimplantation und Sofortversorgung in der Oberkieferfront. Z Zahnärztl Implantol 21, 258-272

Harvey BV (2007): Optimizing the esthetic potential of implant restorations through the use of immediate implants with immediate provisionals. J Periodontol $\underline{78,770-775}$ 
Henriksson CH, Stermer EM, Aass AM, Sandvik L, Moystad A (2008): Comparison of the reproducibility of storage phosphor and film bitewings for assessment of alveolar bone loss. Acta Odontol Scand 66, 380-385

Hermann JS, Cochran DL, Nummikoski PV, Buser D (1997): Crestal bone changes around titanium implants. A radiographic evaluation of unloaded nonsubmerged and submerged implants in the canine mandible. J Periodontol 68, 1117-1130

Heydecke G, Richter E, Seedorf H: Wissenschaftliche Mitteilung der Deutschen Gesellschaft für zahnärztliche Prothetik und Werkstoffkunde (DGZPW): Festsitzender Zahnersatz für zahnbegrenzte Lücken. DGZMK, Düsseldorf 2008

Holm-Pedersen P, Lang NP, Müller F (2007): What are the longevities of teeth and oral implants?. Clin Oral Implants Res 18, 15-19

Holst S, Blatz MB, Hegenbarth E, Wichmann M, Eitner S (2005): Prosthodontic considerations for predictable implant esthetics in the anterior maxilla. J Oral Maxillofac Surg 63, 89-96

Jahn M, d'Hoedt B (1992): Zur Definition des Erfolges bei dentalen Implantaten. Z Zahnärztl Implantol 8 , 221-226

Jemt T (1997): Regeneration of gingival papillae after single-implant treatment. Int J Periodont Rest Dent 17, 326-333

Jemt T, Lekholm U (2003): Measurments of buccal tissue volumes at single-implant restorations after lokal bone grafting in maxillas: a 3-year clinical prospective study case series. Clin Implant Dent Rel Res $\underline{5}, 63-70$

Jivraj S, Chee WWL (2006): Treatment planning of implants in the aesthetic zone. Br Dent J 22, 77-89

Jivraj S, Reshad M, Chee WWL (2005): Critical appraisel. Immediate loading of implants in the esthetic zone. J Esthet Restor Dent 17, 320-325

Joudzbalys G, Wang HL (2007): Soft and hard tissue assessment of immediate implant placement: a case series. Clin Oral Implants Res $\underline{18}$, 237-243

Joudzbalys G, Wang HL (2010): Socket morphology-based treatment for implant esthetics: a pilot study. Int J Oral Maxillofac Implants 25, 970-978

Jung RE, Pjetursson BE, Glauser R, Zembic A, Zwahlen M, Lang NP (2008): A systematic review of the 5-year survival an complication rates of implant-supported single crowns. Clin Oral Implants Res 19, 119-130 
Kan JY, Rungcharassaeng K, Lozada JL, Zimmermann G (2011): Facial gingival tissue stability following immediate placement and provisionalization of maxillary anterior single implants: a 2- to 8- year follow-up. Int J Oral Maxillofac Implants 26, 179-187

Kerschbaum T (2004): Langzeitüberlebensdauer von Zahnersatz. Quintessenz 55, 11131126

Kerschbaum T, Haastert B, Marinello CP (1996): Risk of debonding in three-unit resin-bonded fixed partial denture. J Prosthet Dent 75, 248-25

Kohlmeyer B, Baumann A, Behneke N, Scheller H (2004): Verweilwahrscheinlichkeit und Einflussfaktoren für das Verlustrisiko von Adhäsivbrücken-Eine 15-Jahres-Studie. Dtsch Zahnärztl Z 59, 428-434

Kok IJD, Chang SS, Moriarty JD, Cooper LF (2006): A retrospective analysis of periimplant tissue responses at immediate load/provisionalized mocrothreaded implants. Int J Oral Maxillofac Implants 21, 405-412

Kourtis S, Psarri C, Andritsakis P, Doukoudakis A (2007): Provisional restorations for optimizing esthetics in tanterior maxillary implants: a case report. J Esthet Restor Dent $\underline{19}, 6-17$

Koutrach M, Nimmo A (2010): Preservation of existing soft-tissue contours in the transition from tooth to an implant restoration in the esthetic zone using a flapless approach: a clinical report. Int J Prosthodont 19, 391-396

Lang NP, Adler R, Joss A, Nyman S (1990): Absence of bleeding on probing. An indicator of periodontal stability. J Clin Periodontol, 17, 714-721

Lang NP, Wetzel AC, Stich H, Caffesse RG (1994): Histologic probe penetration in healthy and inflamed peri-implant tissues. Clin Oral Implants Res $\underline{5}, 191-201$

Laviv A, Levin L, Usiel Y, Schwartz-Arad D (2010): Survival of immediately provisionalized dental implants: a case control study with up to 5 year follow-up. Clin Implant Dent Relat Res $\underline{12}, 23-27$

Ledermann PD (1979)): Bar-prosthetic management of the edentulous mandible by means of plasma-coated implantation with titanium screws. Dtsch Zahnärztl Z $\underline{34}$, 907-911

Levi A, Psoter WJ, Agar JR, Reisine ST, Taylor TD (2003): Patient self-reported satisfaction with maxillary anterior dental implant treatment. J Oral Maxillofac Surg $\underline{18}, 113-120$ 
Levin BP (2011): Immediate temporization of immediate implants in the esthetic zone: evaluating survival and bone maintenance. Compend Contin Educ Dent 32, 52-56

Lindeboom JA, Frenken JW, Dubois L, Frank M, Abbink I, Kroon FH (2006): Immediate loading versus immediate provisionalization of maxillary single-tooth replacements: a prospective randomized study with BioComp implants. J Oral Maxillofac Surg $\underline{64}$, 936-942

Locante WM (2001): The nonfunctional immediate provisional in immediate extraction sites: a technique to maximize esthetics. Implant Dent 10, 254-258

Locante WM (2004): Single-tooth replacements in the esthetic zone with an immediate function implant: a preliminary report. J Oral Implantol 30, 369-375

Locker D (1998): Patient-based assessment of the outcomes of implant therapy: a review of the literature. Int J Prosthodont 11, 453-461

Lorenzoni M, Pertl C, Zhang K, Wimmer G, Wegschneider WA (2003): Immediate loading of single-tooth implants in the anterior maxilla. Preliminary results after one year. Clin Oral Implants Res $\underline{14}$, 180-187

Malinski F: Klinische Resultate nach Sofortbelastung dentaler Implantate unter Anwendung kortikaler Mikrofixation. Med. Diss. Göttingen 2011

Malo P, Friberg B, Polizzi G, Gualini F, Vighagen T, Rangert B (2003): Immediate and early function of Branemark System implants placed in the esthetic zone: a 1-year prospective clinical multicenter study. Clin Implant Dent Rel Res 5, 37-46

Meijer HJA, Steen WHA, Bosman F (1993): A comparison of methods to assess marginal bone height around endosseous implants. J Clin Periodontol 20, 250-253

Meijndert L, Meijer HJA, Stellingsma K, Stegenga B, Raghoebar GM (2007): Evaluation of aesthetics of implant-supported single-tooth replacements using different bone augmentation procedures: a prospective randomized clinical study. Clin Oral Implants Res $\underline{18}, 715-719$

Meredith N (1998): Assessment of implant stability as a prognostic determinant. In J Prosthodont $\underline{11}, 491-501$

Meyer U, Joos U, Mythili J, Stamm T, Hohoff A, Fillies T, Stratmann U, Wiesmann HP (2004): Ultrastructural characterization of the implant/bone interface of immediately loaded dental implants. Biomaterials 25, 959-1967 
Meyle J, Mühlbradt L.(1986): Psychologische Aspekte bei der Integration TübingerImplantate in der Sinnessphäre der Mundhöhle. Z Zahnärztl Implantol 4, 211-216

Mijiritsky E, Mardinger O, Mazor Z, Chaushu G (2009): Immediate provisionalization of single-tooth implants in fresh extraction-sites at the maxillary esthetic zone: up to 6 years follow-up. Implant Dent 18, 326-333

Misch CE (1995): Early crestal bone loss etiology and its effect on treatment planning for implants. Postgrad Dent 2 , 3-17

Misch CE, Perel ML, Wang H-L, Sammartino G, Galindo-Moreno P, Trisi P, Steigmann M, Rebaudi A, Palti A, Pikos M, Schwartz-Arad D, Choukroun J, Gutierrez-Perez J-L, Marenzi G, Calacanis DK (2008): Implant Success, Survival, and Failure: The International Congress of Oral Implantologists (ICOI) Pisa Consensus Conference. Implant Dent $\underline{17}, 5-15$

Naert I, Quirynen N, van Steenberghe D, Darius P (1992): A six-year prosthodontic study of 509 consecutively inserted implants for the treatment of partial edentulism. J Prosthet Dent 67, 236-245

Neukam F: Wissenschaftliche Stellungnahme: Lebenserwartung von Implantaten und Implantatlager. DGZMK, Düsseldorf 2000

Noelken R, Morbach T, Kunkel M, Wagner W (2007): Immediate function with NobelPerfect implants in the anterior dental arch. Int J Periodont Rest Dent 27, 277-285

Oh TJ, Shotwell J, Billy E, Byun HY, Wang HL (2007): Flapless implant surgery in the esthetic region: advantages and precautions. Int J Periodont Rest Dent 27, 27-33

Olive J, Aparicio C (1990): Periotest method as a measure of osseointegrated oral implant stability. Int J Oral Maxillofac Implants 5, 390-400

Park JB (2010): The evaluation of digital panoramic radiographs taken for implant dentistry in the daily practice. Med Oral Patol Oral Cir Bucal 15, e 663-666

Palattella P, Torsello F, Cordaro L (2008): Two-year prospective clinical comparison of immediate replacement vs. immediate resoration of single tooth in the esthetic zone. Clin Oral Implants Res 19, 1148-1153

Pjetursson BE, Lang NP (2008): Prosthetic treatment planing on the basis of scientific evidence. J Oral Rehabil $\underline{35}, 72-79$

Raes F, Cosyn J, Crommelinck E, Coessens P, Bruyn HD (2011): Immediate and conventional single implant treatment in the anterior maxilla: 1-year results of a case series on hard and soft tissue responses and aesthetics. J Clin Periodontol 38, 385-394 
Rammelsberg P, Behr M, Pospiech B, Gernet W, Handel G, Toutenburg H (1995): Erweiterte Indikation adhäsiver Restaurationen als ästhetische und substanzschonende Alternative zu konventionellen Brücken. Dtsch Zahnärztl Z 50, 224-227

Randow K, Ericsson I, Nilner K, Petersson A, Glantz P-O (1999): Immediate functional loading of Brånemark dental implants: An 18-month clinical follow-up study. Clin Oral Implants Res $\underline{10}, 8-15$

Ribeiro FS, Pontes AEF, Marcantonio E, Piattelli A, Netto RJB (2008): Successrate of immediate nonfunctional loaded single-tooth implants: immediate versus delayed implantation. Implant Dent $\underline{17}, 109-117$

Richter E-J (2005): Implantologie in der Zahnheilkunde. Dtsch Zahnärztl Z 60, 915-916

Roos J, Sennerby L, Lekholm U, Jemt T, Gröndahl K, Albrektsson T (1997): A qualitative and quantitative method for evaluating implant success: a 5-year retrospective analysis of the Brånemark implant. Int J Oral Maxillofac Implants $\underline{12}, 504-514$

Rouck TD, Collys K, Cosyn J (2008): Immediate single-tooth implants in the anterior maxilla: a 1-year case cohort study on hard and soft tissue response. J Clin Periodontol $\underline{35}, 649-657$

Rouck TD, Collys K, Wyn I, Cosyn J (2009): Instant provisionalization of immediate single-tooth implants is essential to optimize esthetic treatment outcome. Clin Oral Implants Res $\underline{20}, 566-570$

Sammartino G, Marenzi G, di Lauro AE, Paolantoni G (2007): Aesthetics in oral implantology: biological, clinical, surgical and prosthetic aspects. Implant Dent $\underline{16}$, $54-65$

Schliephake: Empfehlungen der DGI: Sofortbelastung - Sofortversorgung. DGI, München 2004

Schnitman PA, Shulman LB (1979): Recommendations of the consensus development conference on dental implants. J Am Dent Assoc 98, 373-377

Smet E, Jacobs R, Gijbels F, Neart I (2006): The accuracy and reliability of radiographic methods for the assessment of marginal bone level around oral implants. J Periodotology 77, 152-160

Snauwaert K, Duyck J, van Steenberghe D, Quirynen M, Naert I (2000): Time dependent failure rate and marginal bone loss of implantat supported prostheses: a 15-year follow-up study. Clin Oral Investig $\underline{4}, 13-20$ 
Somanathan RV, Simunek A, Bukac J, Brazda T, Kopecka D (2007): Soft tissue esthetics in implant dentistry. Acta Med 50, 183-186

Stark H, Holste T, Keßler B (1994): Klebebrücken- Eine 10-Jahresstudie. Dtsch Zahnärztl Z $\underline{49}, 266-270$

Tan K, Pjeturrson BE, Lang NP, Chan ES (2004): A systematic review of the survival and complication rates of fixed partial dentures (FDPs) after observation period of at least 5 years. Clin Oral Implants Res $\underline{15}$, 654-666

Tsirlis AT (2005): Clinical evaluation of immediate loaded upper anterior single implants. Implant Dent 14, 94-103

Turkyilmaz I, Suarez JC (2009): An alternative method for flapless implant placement and an immediate provisional crown: a case report. J Contemp Dent Pract 10, 89-95

Valentini P, Abensur D, Albertini JF, Rocchesani M (2010): Immediate provisionalization of single extraction-site implants in the esthetic zone: a clinical evaluation. Int J Periodont Rest Dent 30, 41-51

Wang HL, Ormianer Z, Palti A, Perel ML, Trisi P, Sammartino G (2006): Consensus conference on immediate loading: the single tooth and partial edentulous areas. Implant Dent $\underline{15}, 324-333$

Weber HP, Morton D, Gallucci GO, Roccuzzo M, Cordaro L, Grutter L (2009): Consensus statements and recommended clinical procedures regarding loading protocols. Int J Oral Maxillofac Implants 24, 180-183

Wheeler SL (2007): Implant complications in the esthetic zone. J Oral Maxillofac Surg 65, 93-102

Wheeler Sl, Vogel RE, Casellini R (2000): Tissue preservation and maintenance of optimum esthetics: a clinical report. Int J Oral Maxillofac Implants 15, 265-271

Zafiropoulos GG, Deli G, Bartee BK, Hoffmann O (2010): Single-tooth implant placement and loading in fresh and regenerated extraction sockets. Five-year results: a case series using two different implant designs. J Periodontol 81, 604-615 


\section{Danksagung}

Zunächst möchte ich meinem Doktorvater, Herrn Prof. Dr. Dr. Wilfried Engelke, für die Bereitstellung und Betreuung der Studie danken sowie für seine Hilfsbereitschaft bei Fragen oder Problemen.

Darüberhinaus danke ich auch ganz besonders dem Praxisteam der Praxis für zahnärztliche Chirurgie von Dr. Stefan Möller, dessen Unterstützung und Hilfsbereitschaft die Durchführung der Studie ermöglichten. 


\section{Lebenslauf}

Am 25. September 1984 wurde ich, Victoria Elisabeth Rost, als zweites Kind von Herrn Detlef Rost und Frau Marion Rost, geb. Marsh, in Hamburg geboren. Von 1991 bis 1995 besuchte ich die Grundschule Klein Flottbecker Weg. Von 1995 bis 2004 besuchte ich das Gymnasium Othmarschen in Hamburg, wo ich 2004 die allgemeine Hochschulreife erlangte.

Im Wintersemester 2004/05 begann ich das Studium der Zahnmedizin an der GeorgAugust-Universität in Göttingen, welches ich Ende 2010 mit dem Staatsexamen erfolgreich abschloss. Die Approbation erhielt ich im Januar 2011. Im Januar 2011 begann ich mit der Promotion unter der Leitung von Prof. Engelke in der Abteilung für Mund-, Kiefer- und Gesichtschirurgie der Universitätsmedizin Göttingen. Seit Herbst 2011 arbeite ich als Assistenzzahnärztin in einer zahnärztlichen Praxis in Göttingen. 